\title{
21. ACCUMULATION OF MARINE AND TERRIGENOUS ORGANIC CARBON AT UPWELLING SITE 658 AND NONUPWELLING SITES 657 AND 659: IMPLICATIONS FOR THE RECONSTRUCTION OF PALEOENVIRONMENTS IN THE EASTERN SUBTROPICAL ATLANTIC THROUGH LATE CENOZOIC TIMES 1
}

\author{
R. Stein, ${ }^{2}$ H. L. ten Haven,${ }^{3}$ R. Littke, ${ }^{3}$ J. Rullkötter,${ }^{3}$ and D. H. Welte ${ }^{3}$
}

\begin{abstract}
Detailed organic geochemical investigations have been performed on sediment samples from upwelling Site 658 and nonupwelling Sites 657 and 659 . The major objective of this study has been the relationship between organic carbon accumulation and paleoclimatic and paleoceanographic conditions in the upwelling area off northwest Africa during late Cenozoic times. The study is based on results from organic carbon, nitrogen, and hydrogen analyses, Rock-Eval pyrolysis, kerogen microscopy, gas chromatography, and gas chromatography/mass spectrometry.

In general, nonupwelling Sites 657 and 659 are characterized by low organic carbon values of less than $0.5 \%$. At Site 657 , four events of high organic carbon deposition (total organic carbon of $1 \%-3 \%$ ) occur and represent turbidites and a slump interval.

The upper Pliocene to Pleistocene sediments of upwelling Site 658 display high organic carbon contents of $0.5 \%-4 \%$, with higher contents concentrated in the upper Pliocene. Accumulation rates of organic carbon vary between 0.1 and $0.5 \mathrm{gC} \cdot \mathrm{cm}^{-2} \cdot 1000 \mathrm{yr}^{-1}$, with maximum values between 3.5 and $3.1 \mathrm{Ma}$. Short-term cyclic ("Milankovitch-type") variations in organic carbon accumulation suggest climate-controlled mechanisms causing these fluctuations.

The quality of organic matter at Site 658 is a mixture of kerogen type II and III, with a dominance of the marine type. This is indicated by high hydrogen-index values of $200-400 \mathrm{mgHC} / \mathrm{gC}$, low $\mathrm{C} / \mathrm{N}$ ratios of $5-15$, atomic $\mathrm{H} / \mathrm{C}$ ratios of 1.0-1.5, and high amounts of marine macerals (alginite and liptodetrinite).

We have estimated paleoproductivity for Sites 658 and 659 based on the amount of marine organic carbon. At open-marine Site 659 , mean paleoproductivity varies between 20 and $50 \mathrm{gC} \cdot \mathrm{m}^{-2} \cdot \mathrm{yr}^{-1}$. At Site 658 , mean paleoproductivity reaches high values of 160 to $320 \mathrm{gC} \cdot \mathrm{m}^{-2} \cdot \mathrm{yr}^{-1}$, very similar to those recorded in modern upwelling areas. The changes in productivity off northwest Africa are linked to changes in nutrient supply caused by both upwelling and fluvial input.

The change from a dominantly humid climate to one characterized by fluctuations between humid and fully arid climates in northwest Africa occurs between 3.1 and 2.45 Ma.
\end{abstract}

\section{INTRODUCTION}

During Leg 108 of the Ocean Drilling Program (ODP), Sites 657 to 661 were drilled on the northwest African continental margin between $21^{\circ} \mathrm{N}$ and $10^{\circ} \mathrm{N}$ at water depths between about 2270 and $4330 \mathrm{~m}$ (Fig. 1). One of the major objectives of Leg 108 was to investigate the evolution of Cenozoic paleoclimate and paleoceanography in the eastern subtropical Atlantic. This study concentrates on the quantity and quality of organic matter deposited at Sites 657, 658, and 659. Detailed studies of organofacies types in marine sediments may provide important information about the provenance of the organic matter, its mode of transportation, and the depositional environment (Arthur et al., 1984; Rullkötter and Mukhopadhyay, 1986; Stein et al., 1986; Summerhayes, 1986). This information is useful for a reconstruction of paleoclimate and paleoceanic circulation in the Atlantic Ocean.

Site $657\left(21^{\circ} 19.89^{\prime} \mathrm{N}, 2^{\circ} 56.93^{\prime} \mathrm{W}\right.$; water depth, $\left.4227 \mathrm{~m}\right)$ is located on the lower continental rise $380 \mathrm{~km}$ west of Cap Blanc, outside the present-day influence of coastal upwelling (Fig. 1). The 178-m sediment sequence of Miocene to Ho-

\footnotetext{
${ }^{1}$ Ruddiman, W. S., Sarnthein, M., et al., 1989. Proc. ODP, Sci. Results, 108: College Station, TX (Ocean Drilling Program).

2 Institut für Geowissenschaften und Lithosphärenforschung (IGL), Universität Giessen, Senckenbergstrasse 3, 6300 Giessen, Federal Republic of Germany.

${ }^{3}$ Institut für Erdöl und Organische Geochemie (ICH-5), KFA Jülich, Postfach 1913, D-5170 Jülich, Federal Republic of Germany.
}

locene age recovered can be divided into two lithologic intervals. The Pliocene to Pleistocene interval consists of light-colored nannofossil ooze alternating with darker colored greenish gray, silt-bearing ooze. Two distinct slumps and several turbiditic sequences are intercalated (Ruddiman, Sarnthein, et al., 1988; Faugères et al., this vol.). The late Miocene interval, separated from the overlying interval by a hiatus of about 1.5 m.y. (Fig. 2), is dominated by brownish nannofossilbearing and barren clay/silty clay. The sedimentation rates at Site 657 vary between 20 and $45 \mathrm{~m} / \mathrm{m}$.y. in the PliocenePleistocene and between 2.5 and $7 \mathrm{~m} / \mathrm{m} . \mathrm{y}$. in the Miocene (Fig. 2; Ruddiman, Sarnthein, et al., 1988).

Site $658\left(20^{\circ} 44.95^{\prime} \mathrm{N}, 18^{\circ} 34.85^{\prime} \mathrm{W}\right.$; water depth, $\left.2263 \mathrm{~m}\right)$ was drilled on the continental slope $160 \mathrm{~km}$ west of Cap Blanc, underneath a major coastal upwelling cell (Fig. 1; Shaffer, 1976; Tomczak and Hughes, 1980). The 300-m sequence of lower Pliocene to Holocene hemipelagic sediments is characterized by cyclic changes between light nannofossil ooze and olive to gray calcareous, siliceous, and siliciclastic sediments. Organic carbon contents reach values of up to $4 \%$. Mean sedimentation rates vary between 72 and $147 \mathrm{~m} / \mathrm{m}$.y. (Fig. 2).

Site $659\left(18^{\circ} 04.63^{\prime} \mathrm{N}, 21^{\circ} 01.57^{\prime} \mathrm{W}\right.$; water depth, $\left.3070 \mathrm{~m}\right)$ is located on top of the smooth Cape Verde Plateau, northeast of the Cape Verde Islands (Fig. 1). The 273.8-m sequence consists of Miocene to Holocene pelagic sediments characterized by cyclic changes between light gray nannofossil/foraminifer-nannofossil ooze and yellowish brown silty nannofossil ooze. The amplitude of variation is less pronounced in the 


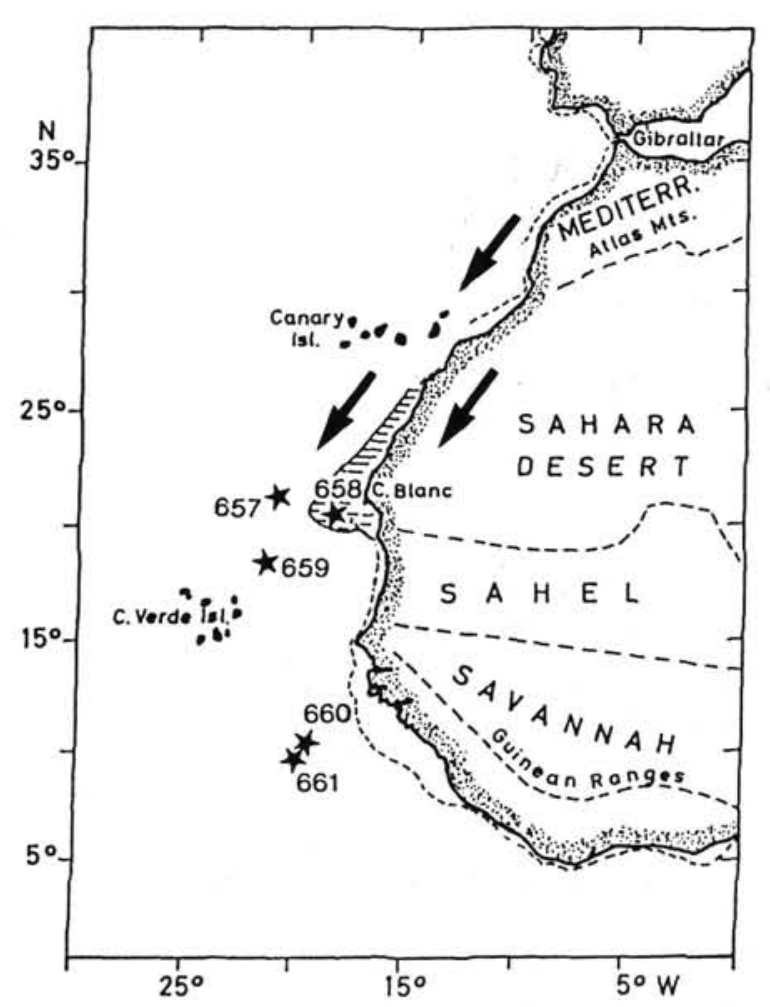

Figure 1. Location of ODP Sites $657,658,659,660$, and 661 . Hatched area marks upwelling off northwest Africa. Black arrows indicate northeast trade winds.

early Pliocene and strongest in the middle/early Miocene. Sedimentation rates vary between $30 \mathrm{~m} / \mathrm{m}$.y. (Pliocene-Pleistocene) and $4 \mathrm{~m} / \mathrm{m}$.y. (Miocene) (Fig. 2).

\section{FACTORS CONTROLLING DEPOSITION OF ORGANIC-CARBON-RICH SEDIMENTS}

In general, modern marine sediments are organic carbon lean rather than organic carbon rich. A typical recent deep-sea sediment has an organic carbon content as low as about $0.2 \%$ (McIver, 1975). However, intervals are known in the geological record in which organic-carbon-rich sediments are much more common and widespread, for example, the Cretaceous black shales in the Atlantic Ocean (Schlanger and Jenkyns, 1976; Arthur et al., 1984; Stein et al., 1986). The deposition of these organic-carbon-rich sediments requires special environmental conditions, the most important of which are the following:

1. oxygen-deficient environments resulting in increased preservation of organic carbon (e.g., the modern Black Sea; Degens and Ross, 1974);

2 . areas characterized by high productivity of organic matter (e.g., upwelling areas; Thiede and Suess, 1983);

3 . areas with an increased supply of terrigenous organic matter (e.g., Baffin Bay; Stein et al., 1989);

4. areas with high bulk sedimentation rates resulting in the rapid burial of organic matter (e.g., in turbidites; Arthur et al., 1984).

Three of these factors, the influences of high oceanic productivity, supply of terrigenous organic matter, and turbidites, can be studied in the sediments off northwest Africa. Thus, not only should investigations of these Neogene to Quaternary sediments yield information about the evolution of the paleoenvironment off northwest Africa, but the results of these investigations may also give a more general model for the deposition of organic-carbon-rich sediments in upwelling environments. This might help to understand the origin of Cretaceous black shales and other fossil organic-carbon-rich strata.

\section{METHODS}

The organic geochemical methods used in this paper are summarized in Figure 3.

Organic and inorganic carbon and total nitrogen were determined using a HERAEUS CHN-Analyzer. In addition, several carbon analyses were performed with a LECO IR-112 Carbon Analyzer (for details and a comparison of organic carbon data derived from both instruments, see Stein et al., 1989). For a selected set of samples, hydrogen and carbon were measured in kerogen concentrates by means of the HERAEUS CHN-Analyzer in order to obtain atomic hydrogen/carbon $(\mathrm{H} / \mathrm{C})$ ratios.

Rock-Eval pyrolysis was performed according to the method described by Espitalié et al. (1977).

Kerogen microscopy analyses were carried out after kerogen concentration by treating the samples successively with $\mathrm{HCl}$ and $\mathrm{HF}$ in order to remove carbonates and silicates, respectively (Durand, 1980). The washed and dried material was embedded in a resin. After polishing the resulting blocks, maceral analyses were performed on these blocks by a two-scan method in reflected light and in a fluorescence mode (cf. Littke, 1987). The macerals were classified according to the nomenclature described by Stach et al. (1982) and Hutton et al. (1980). For maturity assessments, vitrinite reflectances were measured on particles larger than $10 \mu \mathrm{m}(\lambda=546 \mathrm{~nm}$, oil immersion; see Stach et al., 1982).

Gas chromatography and gas chromatography/mass spectrometry of extracts from selected samples were performed as described by ten Haven et al. (this vol.).

Mass accumulation rates (MAR) were calculated according to van Andel et al. (1975) with physical property and sedimentation rate data from Ruddiman, Sarnthein, et al. (1988), as follows:

$$
\operatorname{MAR}\langle\mathrm{TOC}\rangle=(\mathrm{TOC} / 100) \cdot \mathrm{LSR} \cdot[\mathrm{WBD}-1.026(\mathrm{PO} / 100)],
$$

where MAR $=$ mass accumulation rates $\left(\mathrm{g} \cdot \mathrm{cm}^{-2} \cdot 1000 \mathrm{yr}^{-1}\right)$;

TOC $=$ total organic carbon $(w t \%)$;

LSR $=$ linear sedimentation rates $\left(\mathrm{cm} \cdot 1000 \mathrm{yr}^{-1}\right)$;

WBD $=$ wet-bulk density $\left(\mathrm{g} \cdot \mathrm{cm}^{-3}\right)$; and

$\mathrm{PO}=$ porosity $(\%)$.

For Site 658, we used the detailed record of sedimentation rates based on oxygen isotopes derived by Sarnthein and Tiedemann (this vol.).

Surface-water paleoproductivity was estimated with the following equation:

$\mathrm{P}=5.31 \cdot\left[\mathrm{C} \cdot\left(\mathrm{WBD}-1.026 \cdot \mathrm{PO} \cdot 10^{-2}\right)\right]^{0.71} \cdot \mathrm{SR}^{0.07} \cdot \mathrm{DEP} \mathrm{P}^{0.45}$,

where $\mathrm{P}=$ paleoproductivity $\left(\mathrm{gC} \cdot \mathrm{m}^{-2} \cdot \mathrm{yr}^{-1}\right)$;

$\mathrm{C}=$ (marine) organic carbon $(\mathrm{wt} \%)$;

WBD $=$ wet-bulk density $\left(\mathrm{g} \cdot \mathrm{cm}^{-3}\right)$;

$\mathrm{SR}=$ sedimentation rate $\left(\mathrm{cm} \cdot 1000 \mathrm{yr}^{-1}\right)$;

$\mathrm{PO}=$ porosity $(\%)$; and

$\mathrm{DEP}=$ paleowater depth of seafloor in meters.

This formula is based on the relationships among organic carbon accumulation rates in surface sediments, sedimentation rates, water depths, and measured (recent) surface-water productivity from the central Pacific, the continental margins off northwest Africa, northwest and southwest America, and the Argentine Basin areas (Müller and Suess, 1979; Betzer et al., 1984). For a detailed deduction and limits on the use of the equation, see Stein (1986).

The amounts of quartz and nonquartz siliciclastics (mainly clay minerals and feldspars) are determined by X-ray diffractometry (XRD; method and data list in Tiedemann et al., this vol.). 


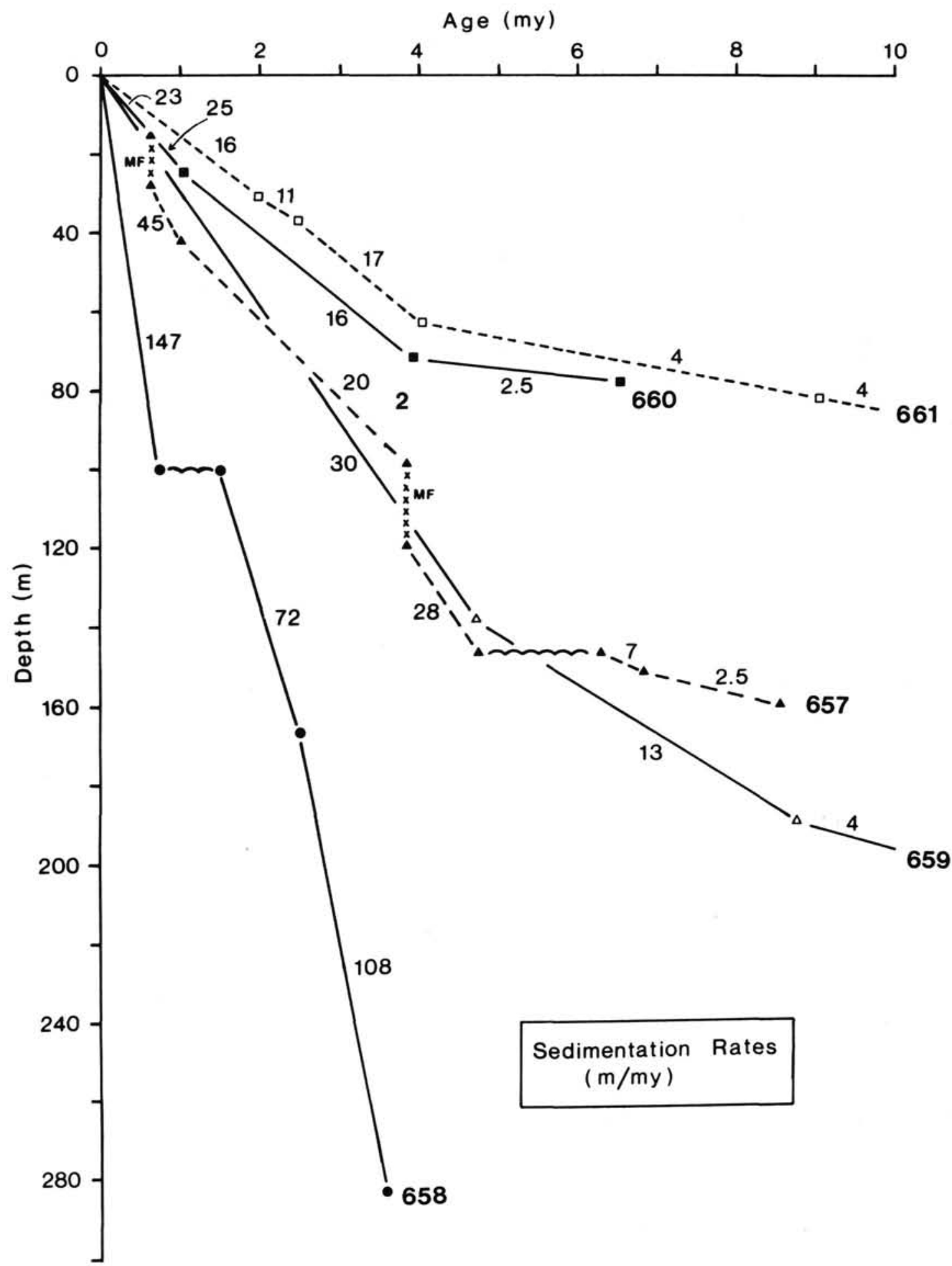

Figure 2. Mean sedimentation rates based on shipboard data (Ruddiman, Sarnthein, et al., 1988).

\section{RESULTS}

\section{Quantity of Organic Matter}

The entire sediment sequence of upwelling Site 658 is characterized by high TOC values ranging between about $0.5 \%$ and $4 \%$ (Fig. 4 and Table 1). Such values are higher than those recorded in normal open-marine environments. During upper Pliocene times (below $100 \mathrm{mbsf}$ ), TOC values vary between $1 \%$ and $4 \%$; during the Pleistocene (above the hiatus at about $100 \mathrm{mbsf}$, however, lower TOC contents of $0.5 \%-2.5 \%$ are typical (Fig. 4). Throughout the entire section, high-amplitude, short-term fluctuations in organic carbon occur. These cyclic variations have periods between about 20,000 to $100,000 \mathrm{yr}$, as shown in greater detail in Figure 4 for three time intervals. The absolute maximum TOC values are separated by about $10 \mathrm{~m}$, which corresponds to about 100,000 yr, based on mean sedimentation rates (Fig. 2). 


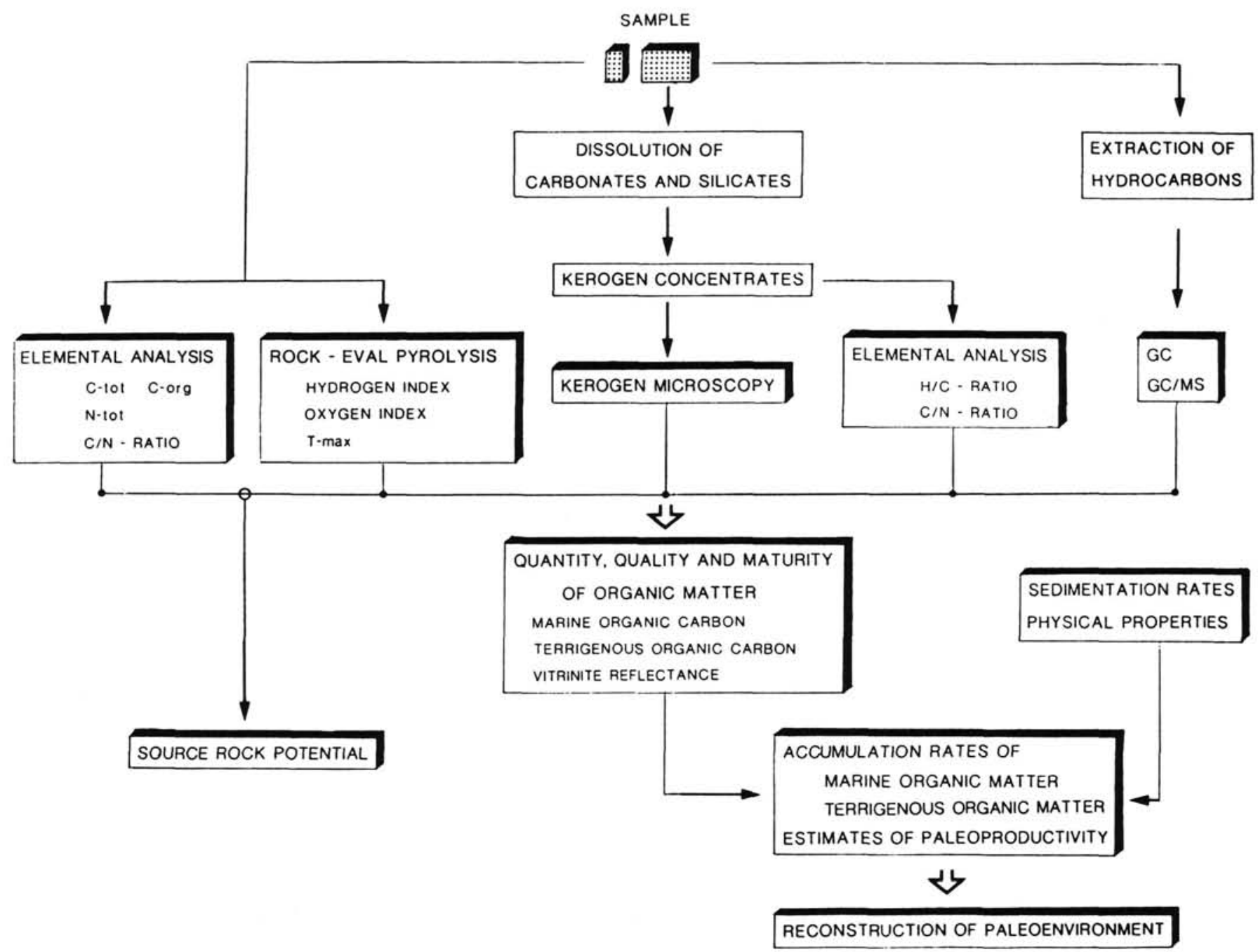

Figure 3. Summary of organic geochemical methods used in this study.

The sediments at nonupwelling Sites 657 and 659 are characterized by low TOC contents, with values between $0.05 \%$ and $0.5 \%$ during Pliocene-Pleistocene times and below $0.1 \%$ during late Miocene times (Fig. 5 and Table 2). These values are typical for modern open-marine environments (McIver, 1975). At Site 657, four events with high TOC contents of $1 \%-3 \%$ are recorded (Fig. 5). Three of these intervals are from turbiditic sequences, and the fourth is from a slump deposit.

Because changes in the percentage values of TOC can result from changes in both organic carbon supply and deposition of mineral matter (mainly carbonate and siliciclastic material), TOC percentage values from Sites 658 and 659 were transformed into mass accumulation rates of organic carbon (see "Methods" section, this chapter). This procedure was not performed on TOC data from Site 657 because of the repeated occurrence of turbidites (cf. Faugères et al., this vol.). With these mass accumulation rates, dilution effects by mineral matter can be excluded, and the organic carbon data can be interpreted in terms of changes in supply and/or preservation of organic matter.

At Site 658 , the accumulation rates of organic carbon vary between 0.05 and $0.5 \mathrm{gC} \cdot \mathrm{cm}^{-2} \cdot 1000 \mathrm{yr}^{-1}$ (Fig. 6). Higher values of $\sim 0.3-0.5 \mathrm{gC} \cdot \mathrm{cm}^{-2} \cdot 1000 \mathrm{yr}^{-1}$ are found between 3.5 and $3.1 \mathrm{Ma}$, and between 2.4 and 1.6 Ma values between 0.1 and $0.2 \mathrm{gC} \cdot \mathrm{cm}^{-2} \cdot 1000 \mathrm{yr}^{-1}$ occur. During the last $0.7 \mathrm{Ma}$, high-amplitude variations of organic-carbon accumulation rates between 0.05 and $0.35 \mathrm{gC} \cdot \mathrm{cm}^{-2} \cdot 1000 \mathrm{yr}^{-1}$ are typical (Fig. 6).

At Site 659, mass accumulation rates of organic carbon are one order of magnitude lower (Fig. 6). They vary between 0.003 and $0.01 \mathrm{gC} \cdot \mathrm{cm}^{-2} \cdot 1000 \mathrm{yr}^{-1}$ during the last $4.5 \mathrm{Ma}$. In the upper Miocene, extremely low accumulation rates around $0.002 \mathrm{gC} \cdot \mathrm{cm}^{-2} \cdot \mathrm{k} . \mathrm{y} \cdot{ }^{-1}$ have been recorded (Fig. 6).

\section{Quality of the Organic Matter}

To determine the factors controlling the deposition of organic carbon in a specific environment, it is necessary to know the amounts of marine and terrigenous organic carbon. Based on TOC data only, a reconstruction of the depositional history of organic carbon is of very limited significance.

In this study, the amounts of marine and terrigenous organic carbon have been estimated by a joint application of different analytical methods: Rock-Eval pyrolysis, C/N ratios, kerogen microscopy, gas chromatography, and gas chromatography/mass spectrometry. The use of different independent methods to qualify the organic matter is important because factors other than the primary source may also influence the organic matter composition. Thus, additional information 


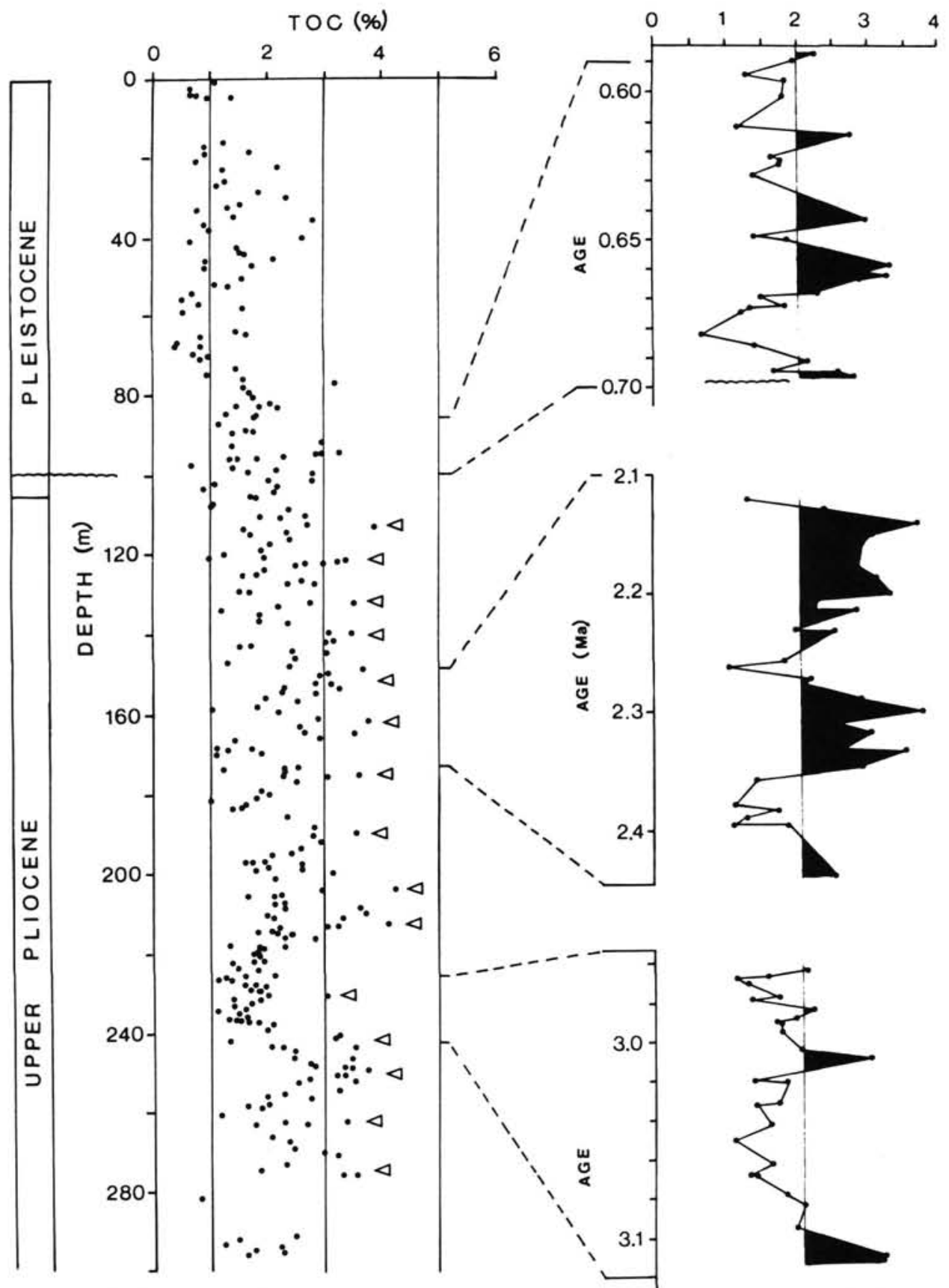

Figure 4. Total organic carbon contents at Site 658 (data of Table 1). Open triangles mark absolute TOC maxima. Based on mean sedimentation rates, the distance between triangles is about $100,000 \mathrm{yr}$. On the right-hand side, three intervals are shown enlarged to indicate short-term fluctuations.

about the stage of maturity (diagenesis) is helpful for the interpretation.

\section{Rock-Eval Pyrolysis}

Hydrogen and oxygen index values derived from Rock-Eval pyrolysis are shown in Figure 7. The data suggest that most of the organic matter in sediments from Site 658 is a mixture of kerogen types II and III, a mixture of marine and terrigenous material. The unusually high oxygen indexes may result from significant amounts of labile organic matter (e.g., sugars, proteins) still present in immature marine sediments. Throughout the entire sequence, most of the hydrogen index values are relatively high and vary between 200 and $400 \mathrm{mgHC} / \mathrm{g} \mathrm{C}_{\text {org }}$ (Fig.
8), suggesting that a major portion of the organic matter may have been derived from marine algae, phytoplankton, zooplankton, and bacteria. Rough estimates of the amount of marine organic carbon based on the hydrogen indexes vary between about $40 \%$ and $80 \%$ (Table 1, col. 7; estimates based on the correlation between hydrogen indexes and maceral composition shown for DSDP sediments in Stein et al., 1986).

In the pelagic sediments of Sites 657 and 659 , hydrogen index values are significantly lower, ranging between about 50 and 120 $\mathrm{mgHC} / \mathrm{g} \mathrm{C}_{\text {org }}$ (Tables 3 and 4; open squares in Fig. 7). The slump deposit sediments at Site 657 are characterized by hydrogen indexes very similar to those measured for sediments from Site 658 (160-320 mgHC/C ${ }_{\text {org }}$; open circles in Fig. 7; Table 3). 
Table 1. Summary of data from Site 658.

\begin{tabular}{|c|c|c|c|c|c|c|c|c|c|c|c|}
\hline $\begin{array}{l}\text { Core, section, } \\
\text { interval }(\mathrm{cm})\end{array}$ & $\begin{array}{l}\text { Depth } \\
\text { (mbsf) }\end{array}$ & $\mathrm{CaCO}_{3}$ & $\mathrm{C}_{\text {tot }}$ & $\mathrm{C}_{\text {org }^{\prime}}$ & $\mathrm{C}_{\text {org }}$ & MOM & $\mathrm{N}_{\text {tot }}$ & $\mathrm{C} / \mathrm{N}$ & HI & OI & $T_{\max }$ \\
\hline \multicolumn{12}{|l|}{$108-658 \mathrm{~A}-$} \\
\hline $1 \mathrm{H}-1,40$ & 0.40 & 30.3 & 6.30 & 3.83 & 2.67 & & 0.29 & 9 & & & \\
\hline $1 \mathrm{H}-1,120$ & 1.20 & 57.5 & 7.99 & & 1.09 & 65 & 0.16 & 7 & 350 & 492 & 409 \\
\hline $1 \mathrm{H}-2,40$ & 1.90 & & 7.04 & & & & 0.23 & & & & \\
\hline $1 \mathrm{H}-2,120$ & 2.70 & 39.3 & 5.39 & & 0.67 & 55 & 0.10 & 7 & 242 & 642 & 415 \\
\hline $1 \mathrm{H}-3,40$ & 3.40 & 36.4 & 5.04 & 1.06 & 0.67 & 45 & 0.07 & 10 & 182 & & \\
\hline $1 \mathrm{H}-3,120$ & 4.20 & 37.7 & 5.26 & & 0.74 & 70 & 0.16 & 5 & 454 & 561 & 420 \\
\hline $1 \mathrm{H}-4,34$ & 4.84 & 39.0 & 5.64 & & 0.96 & 60 & 0.21 & 5 & 285 & 442 & 415 \\
\hline $1 \mathrm{H}-4,40$ & 4.90 & 44.0 & 6.66 & 2.46 & 1.38 & & 0.15 & 9 & & & \\
\hline $3 \mathrm{H}-1,40$ & 15.60 & 46.3 & 7.42 & 3.46 & 1.86 & & 0.18 & 10 & & & \\
\hline $3 \mathrm{H}-1,120$ & 16.40 & 52.8 & 7.57 & & 1.23 & 45 & 0.27 & 5 & 205 & 432 & 409 \\
\hline $3 \mathrm{H}-2,40$ & 17.10 & 45.5 & 6.38 & 1.68 & 0.91 & 55 & 0.10 & 9 & 252 & & 417 \\
\hline $3 \mathrm{H}-3,40$ & 18.60 & 34.4 & 5.83 & 2.60 & 1.71 & 50 & 0.22 & 8 & 220 & & 419 \\
\hline $3 \mathrm{H}-3,120$ & 19.40 & 48.3 & 6.72 & & 0.92 & 60 & 0.20 & 5 & 297 & 527 & 416 \\
\hline $3 \mathrm{H}-4,40$ & 20.10 & & 6.46 & & & & 0.17 & & & & \\
\hline $3 \mathrm{H}-4,120$ & 20.90 & 41.2 & 5.71 & & 0.77 & 55 & 0.13 & 6 & 260 & 492 & 414 \\
\hline $3 \mathrm{H}-5,40$ & 21.60 & & 5.46 & & & & 0.07 & & & & \\
\hline $3 \mathrm{H}-5,140$ & 22.60 & 39.4 & 6.92 & & 2.19 & 65 & 0.17 & 13 & 322 & & 417 \\
\hline $3 \mathrm{H}-6,34$ & 23.04 & 36.4 & 5.61 & & 1.24 & 55 & 0.24 & 5 & 274 & 332 & 414 \\
\hline $3 \mathrm{H}-6,40$ & 23.10 & 25.6 & 4.16 & 1.44 & 1.07 & 30 & 0.08 & 13 & 86 & & \\
\hline $4 \mathrm{H}-1,59$ & 25.29 & 40.3 & 6.56 & 2.90 & 1.73 & 50 & 0.17 & 10 & 213 & & \\
\hline $4 \mathrm{H}-1,120$ & 25.90 & 48.4 & 7.09 & & 1.28 & 65 & 0.18 & 7 & 309 & 355 & 415 \\
\hline $4 \mathrm{H}-2,40$ & 26.60 & 41.3 & 6.65 & 2.89 & 1.70 & & 0.11 & 15 & & & \\
\hline $4 \mathrm{H}-2,120$ & 27.40 & 35.9 & 5.43 & & 1.12 & 65 & 0.14 & 8 & 338 & 426 & 418 \\
\hline $4 \mathrm{H}-3,40$ & 28.10 & & 5.37 & & & & 0.18 & & & & \\
\hline $4 \mathrm{H}-3,120$ & 28.90 & 34.4 & 5.99 & & 1.86 & 60 & 0.25 & 7 & 280 & 245 & 415 \\
\hline $4 \mathrm{H}-4,40$ & 29.60 & 42.8 & 6.59 & 2.55 & 1.46 & & 0.17 & 9 & & & \\
\hline $4 \mathrm{H}-4,120$ & 30.40 & 33.6 & 6.39 & & 2.36 & 60 & 0.33 & 7 & 284 & 287 & 411 \\
\hline $4 \mathrm{H}-5,40$ & 31.10 & 26.8 & 6.24 & 4.13 & 3.02 & & 0.29 & 10 & & & \\
\hline $4 \mathrm{H}-5,120$ & 31.90 & 45.1 & 6.95 & & 1.54 & 65 & 0.23 & 7 & 310 & 329 & 414 \\
\hline $4 \mathrm{H}-6,40$ & 32.60 & 42.6 & 6.45 & 2.33 & 1.34 & 40 & 0.11 & 12 & 168 & & 418 \\
\hline $4 \mathrm{H}-6,120$ & 33.40 & 40.4 & 5.62 & & 0.77 & 55 & 0.11 & 7 & 242 & 503 & 423 \\
\hline $5 \mathrm{H}-1,84$ & 35.02 & 45.8 & 6.92 & & 1.42 & 60 & 0.19 & 8 & 293 & 402 & 424 \\
\hline $5 \mathrm{H}-2,40$ & 36.02 & 22.1 & 5.47 & 3.62 & 2.82 & 40 & 0.19 & 15 & 148 & & 406 \\
\hline $5 \mathrm{H}-2,120$ & 36.82 & 41.4 & 5.87 & & 0.90 & 55 & 0.15 & 6 & 258 & 388 & 422 \\
\hline $5 \mathrm{H}-3,40$ & 37.48 & 39.6 & 6.30 & 2.57 & 1.55 & & 0.15 & 10 & & & \\
\hline $5 \mathrm{H}-3,120$ & 38.28 & 35.2 & 5.20 & & 0.98 & 55 & 0.12 & 8 & 269 & 415 & 415 \\
\hline $5 \mathrm{H}-4,40$ & 39.58 & & 6.13 & & & & 0.13 & & & & \\
\hline $5 \mathrm{H}-5,40$ & 40.49 & 25.4 & 5.67 & 3.51 & 2.62 & 15 & 0.07 & 37 & 68 & & \\
\hline $5 \mathrm{H}-5,120$ & 41.19 & 48.0 & 6.43 & & 0.67 & 60 & 0.15 & 4 & 281 & 510 & 411 \\
\hline $5 \mathrm{H}-6,40$ & 41.85 & 32.1 & 4.87 & 1.50 & 1.02 & & 0.06 & 17 & & & \\
\hline $5 \mathrm{H}-6,120$ & 42.65 & 56.0 & 8.25 & & 1.53 & 65 & 0.19 & 8 & 333 & 341 & 422 \\
\hline $5 \mathrm{H}-7,34$ & 43.27 & 63.5 & 9.09 & & 1.47 & 65 & 0.17 & 9 & 310 & 280 & 420 \\
\hline $5 \mathrm{H}-7,38$ & 43.31 & 50.3 & 8.55 & 5.05 & 2.51 & & 0.23 & 11 & & & \\
\hline $6 \mathrm{H}-1,59$ & 44.29 & & 8.68 & & & & 0.18 & & & & \\
\hline $6 \mathrm{H}-1,84$ & 44.54 & 52.9 & 7.96 & & 1.61 & 60 & 0.13 & 12 & 297 & 336 & 414 \\
\hline $6 \mathrm{H}-2,40$ & 45.60 & 48.2 & 7.91 & 4.11 & 2.13 & 65 & 0.18 & 12 & 353 & & 416 \\
\hline $6 \mathrm{H}-2,120$ & 46.40 & 31.9 & 4.77 & & 0.94 & 45 & 0.15 & 6 & 205 & 412 & 419 \\
\hline $6 \mathrm{H}-3,40$ & 47.10 & 48.7 & 7.60 & 3.43 & 1.76 & 65 & 0.16 & 11 & 343 & & 416 \\
\hline $6 \mathrm{H}-3,120$ & 47.90 & 47.9 & 6.67 & & 0.92 & 60 & 0.12 & 8 & 308 & 441 & 425 \\
\hline $6 \mathrm{H}-5,120$ & 50.90 & 40.4 & 6.42 & & 1.57 & 55 & 0.19 & 8 & 277 & 329 & 416 \\
\hline $6 \mathrm{H}-6,40$ & 51.60 & 45.2 & 7.31 & 3.45 & 1.89 & 60 & 0.16 & 12 & 268 & & \\
\hline $6 \mathrm{H}-6,120$ & 52.40 & 56.0 & 7.83 & & 1.11 & 70 & 0.08 & 14 & 361 & 280 & 420 \\
\hline $6 \mathrm{H}-7,34$ & 52.77 & 25.8 & 4.43 & & 1.33 & 60 & 0.18 & 7 & 286 & 288 & 413 \\
\hline $6 \mathrm{H}-7,40$ & 52.83 & 28.3 & 5.37 & 2.76 & 1.98 & & 0.16 & 12 & & & \\
\hline $7 \mathrm{H}-1,120$ & 54.40 & 45.5 & 6.18 & & 0.72 & 50 & 0.02 & 36 & 247 & 458 & 415 \\
\hline $7 \mathrm{H}-2,40$ & 55.10 & & 6.24 & & & & 0.07 & & & & \\
\hline $7 \mathrm{H}-2,120$ & 55.90 & 37.0 & 4.97 & & 0.53 & 45 & 0.09 & 6 & 172 & 630 & 403 \\
\hline $7 \mathrm{H}-3,40$ & 56.60 & 44.3 & 6.39 & 1.93 & 1.08 & & 0.08 & 14 & & & \\
\hline $7 \mathrm{H}-3,120$ & 57.40 & 53.3 & 7.21 & & 0.81 & 60 & 0.14 & 6 & 288 & 532 & 422 \\
\hline $7 \mathrm{H}-4,40$ & 58.10 & 56.7 & 8.39 & 3.66 & 1.58 & 60 & 0.12 & 13 & 282 & & 414 \\
\hline $7 \mathrm{H}-4,120$ & 58.90 & 47.0 & 6.17 & & 0.53 & 55 & 0.04 & 13 & 268 & 760 & 407 \\
\hline $8 \mathrm{H}-1,40$ & 63.10 & 54.3 & 7.96 & 3.16 & 1.44 & & 0.13 & 11 & & & \\
\hline $8 \mathrm{H}-1,120$ & 63.90 & 42.2 & 6.53 & & 1.47 & 45 & 0.17 & 9 & 182 & 305 & 412 \\
\hline $8 \mathrm{H}-2,40$ & 64.60 & 56.8 & 8.46 & 3.81 & 1.65 & 60 & 0.15 & 11 & 297 & & 413 \\
\hline $8 \mathrm{H}-2,118$ & 65.38 & 49.8 & 6.83 & & 0.85 & 65 & 0.09 & 9 & 345 & 578 & 418 \\
\hline $8 \mathrm{H}-3,40$ & 66.10 & & 5.66 & & & & 0.11 & & & & \\
\hline $8 \mathrm{H}-3,115$ & 66.85 & 41.0 & 5.36 & & 0.44 & 60 & 0.08 & 6 & 289 & 641 & 405 \\
\hline $8 \mathrm{H}-4,40$ & 67.60 & 30.5 & 4.06 & 0.57 & 0.40 & 50 & 0.04 & 10 & 225 & & \\
\hline $8 \mathrm{H}-4,120$ & 68.40 & 57.6 & 7.77 & & 0.86 & 65 & 0.17 & 5 & 344 & 628 & 412 \\
\hline $8 \mathrm{H}-5,40$ & 69.10 & 49.9 & 6.82 & 1.66 & 0.83 & 45 & 0.08 & 10 & 183 & & \\
\hline $8 \mathrm{H}-5,120$ & 69.90 & 50.0 & 6.72 & & 0.72 & 60 & 0.06 & 12 & 306 & 531 & 422 \\
\hline $8 \mathrm{H}-6,40$ & 70.60 & 55.8 & 7.65 & 2.16 & 0.95 & 50 & 0.08 & 12 & 241 & & 410 \\
\hline $8 \mathrm{H}-6,81$ & 71.01 & 68.0 & 9.01 & & 0.85 & 70 & 0.06 & 14 & 379 & 406 & 421 \\
\hline $9 \mathrm{H}-1,40$ & 72.60 & & 6.25 & & & & 0.19 & & & & \\
\hline $9 \mathrm{H}-1,120$ & 73.40 & 54.9 & 8.04 & & 1.45 & 65 & 0.07 & 21 & 317 & 332 & 420 \\
\hline
\end{tabular}


Table 1 (continued).

\begin{tabular}{|c|c|c|c|c|c|c|c|c|c|c|c|}
\hline $\begin{array}{l}\text { Core, section, } \\
\text { interval }(\mathrm{cm})\end{array}$ & $\begin{array}{l}\text { Depth } \\
\text { (mbsf) }\end{array}$ & $\mathrm{CaCO}_{3}$ & $\mathrm{C}_{\text {tot }}$ & $\mathrm{C}_{\text {org' }}$ & $\mathrm{C}_{\text {org }}$ & MOM & $\mathrm{N}_{\text {tot }}$ & $\mathrm{C} / \mathrm{N}$ & $\mathrm{HI}$ & OI & $\mathrm{T}_{\max }$ \\
\hline $9 \mathrm{H}-2,52$ & 74.22 & 33.1 & 5.10 & 1.69 & 1.13 & & 0.05 & 23 & & & \\
\hline $9 \mathrm{H}-2,120$ & 74.90 & 62.6 & 8.46 & & 0.95 & 50 & 0.10 & 10 & 238 & 468 & 415 \\
\hline $9 \mathrm{H}-3,40$ & 75.60 & & 8.22 & & & & 0.12 & & & & \\
\hline $9 \mathrm{H}-3,86$ & 76.06 & 52.9 & 7.92 & & 1.57 & 45 & 0.07 & 22 & 203 & 235 & 418 \\
\hline $9 \mathrm{H}-4,40$ & 77.10 & 44.6 & 8.54 & 5.75 & 3.18 & 50 & 0.20 & 16 & 254 & & 414 \\
\hline $9 \mathrm{H}-4,120$ & 77.90 & 44.2 & 6.89 & & 1.59 & 60 & 0.20 & 8 & 291 & 293 & 421 \\
\hline $9 \mathrm{H}-5,40$ & 78.60 & & 8.49 & & & & 0.17 & & & & \\
\hline $9 \mathrm{H}-5,120$ & 79.40 & 52.0 & 7.93 & & 1.69 & 65 & 0.12 & 14 & 356 & 260 & 424 \\
\hline $9 \mathrm{H}-6,40$ & 80.10 & 40.2 & 7.99 & 5.30 & 3.17 & & 0.22 & 14 & & & \\
\hline $9 \mathrm{H}-6,120$ & 80.90 & 48.2 & 7.54 & & 1.76 & 65 & 0.20 & 9 & 330 & 266 & 421 \\
\hline $10 \mathrm{H}-1,40$ & 82.10 & 45.2 & 7.48 & 3.75 & 2.05 & 65 & 0.16 & 13 & 318 & & 416 \\
\hline $10 \mathrm{H}-1,120$ & 82.90 & 60.5 & 8.72 & & 1.46 & 70 & 0.12 & 12 & 397 & 354 & 409 \\
\hline $10 \mathrm{H}-1,139$ & 83.00 & 52.6 & 8.48 & & 2.17 & 65 & 0.15 & 14 & 332 & & 420 \\
\hline $10 \mathrm{H}-2,40$ & 83.60 & 31.7 & 5.67 & 2.73 & 1.86 & 50 & 0.12 & 16 & 234 & & 408 \\
\hline $10 \mathrm{H}-2,120$ & 84.90 & 56.2 & 8.02 & & 1.28 & 55 & 0.16 & 8 & 254 & 298 & 421 \\
\hline $10 \mathrm{H}-3,40$ & 85.10 & 47.3 & 7.49 & 3.45 & 1.82 & 65 & 0.16 & 11 & 310 & & 410 \\
\hline $10 \mathrm{H}-3,120$ & 85.90 & 43.5 & 7.02 & & 1.80 & 50 & 0.19 & 9 & 238 & 311 & 412 \\
\hline $10 \mathrm{H}-4,40$ & 86.60 & & 6.62 & & & & 0.19 & & & & \\
\hline $10 \mathrm{H}-4,120$ & 87.40 & 36.1 & 5.49 & & 1.16 & 55 & 0.15 & 8 & 243 & 402 & 415 \\
\hline $10 \mathrm{H}-5,40$ & 88.10 & 30.7 & 6.45 & 4.00 & 2.77 & & 0.28 & 10 & & & \\
\hline $10 \mathrm{H}-5,118$ & 88.88 & 38.4 & 6.24 & & 1.63 & 55 & 0.23 & 7 & 275 & 329 & 416 \\
\hline $10 \mathrm{H}-5,138$ & 89.00 & 44.3 & 7.09 & & 1.77 & 60 & 0.18 & 10 & 280 & & 421 \\
\hline $10 \mathrm{H}-6,40$ & 89.60 & 48.3 & 7.55 & 3.39 & 1.75 & & 0.16 & 11 & & & \\
\hline $10 \mathrm{H}-6,48$ & 89.68 & 50.9 & 7.51 & & 1.40 & 60 & 0.15 & 9 & 306 & 304 & 418 \\
\hline $10 \mathrm{H}-7,120$ & 91.90 & 28.5 & 6.37 & & 2.95 & 55 & 0.28 & 11 & 275 & 202 & 419 \\
\hline $11 \mathrm{H}-2,24$ & 92.72 & 49.3 & 7.30 & & 1.38 & 60 & 0.21 & 7 & 296 & 341 & 414 \\
\hline $11 \mathrm{H}-2,42$ & 92.80 & 42.6 & 6.94 & 3.19 & 1.83 & & 0.21 & 9 & & & \\
\hline $11 \mathrm{H}-3,42$ & 94.11 & 29.8 & 6.88 & 4.70 & 3.30 & & 0.27 & 12 & & & \\
\hline $11 \mathrm{H}-3,84$ & 94.50 & 29.8 & 6.52 & & 2.95 & 65 & 0.28 & 11 & 369 & & 415 \\
\hline $11 \mathrm{H}-3,96$ & 94.65 & 24.8 & 6.24 & & 3.26 & 65 & 0.47 & 7 & 337 & 208 & 416 \\
\hline $11 \mathrm{H}-3,102$ & 94.70 & 26.9 & 6.10 & & 2.87 & 65 & 0.25 & 11 & 324 & 241 & 417 \\
\hline $11 \mathrm{H}-4,42$ & 95.46 & 17.6 & 4.39 & 2.77 & 2.28 & 50 & 0.19 & 12 & 233 & & 415 \\
\hline $11 \mathrm{H}-4,62$ & 95.66 & 28.9 & 4.96 & & 1.49 & 55 & 0.31 & 5 & 255 & 300 & 417 \\
\hline $11 \mathrm{H}-4,116$ & 96.13 & 46.5 & 7.40 & & 1.82 & & 0.36 & 5 & & & \\
\hline $11 \mathrm{H}-4,120$ & 96.17 & 45.8 & 6.85 & & 1.35 & 65 & 0.22 & 6 & 353 & 361 & 414 \\
\hline $11 \mathrm{H}-5,42$ & 96.69 & 26.8 & 4.31 & 1.49 & 1.09 & & 0.10 & 11 & & & \\
\hline $11 \mathrm{H}-5,120$ & 97.47 & 26.1 & 3.81 & & 0.68 & 80 & 0.07 & 10 & 656 & 706 & 419 \\
\hline $11 \mathrm{H}-6,42$ & 98.00 & 28.0 & 4.76 & 1.95 & 1.40 & 50 & 0.11 & 13 & 234 & & 416 \\
\hline $11 \mathrm{H}-6,120$ & 98.78 & 36.0 & 6.48 & & 2.16 & 55 & 0.20 & 11 & 276 & 257 & 415 \\
\hline $11 \mathrm{H}-7,24$ & 99.25 & 39.4 & 6.39 & & 1.66 & 55 & 0.08 & 21 & 266 & 235 & 426 \\
\hline $11 \mathrm{H}-7,42$ & 99.43 & 38.3 & 7.15 & 4.11 & 2.55 & & 0.22 & 12 & & & \\
\hline $11 \mathrm{H}-7,83$ & 99.80 & & & & 2.79 & 65 & & & 321 & & 420 \\
\hline $11 \mathrm{H}-8,42$ & 100.83 & 30.3 & 4.98 & 1.94 & 1.35 & & 0.05 & 27 & & & \\
\hline $12 \mathrm{H}-1,24$ & 100.90 & 35.7 & 6.30 & & 2.02 & 55 & 0.22 & 9 & 247 & 267 & 414 \\
\hline $12 \mathrm{H}-1,40$ & 101.06 & 29.7 & 6.33 & 3.94 & 2.77 & 55 & 0.27 & 10 & 259 & & 416 \\
\hline $12 \mathrm{H}-2,32$ & 102.20 & 29.3 & 4.60 & & 1.09 & 55 & 0.11 & 10 & 239 & & 417 \\
\hline $12 \mathrm{H}-2,118$ & 102.98 & 28.1 & 5.55 & & 2.18 & 50 & 0.13 & 17 & 222 & 219 & 418 \\
\hline $12 \mathrm{H}-3,50$ & 103.57 & 32.4 & 4.77 & 1.31 & 0.89 & 45 & 0.10 & 9 & 199 & & \\
\hline $12 \mathrm{H}-3,120$ & 104.27 & 32.6 & 6.04 & & 2.13 & 55 & 0.26 & 8 & 262 & 192 & 423 \\
\hline $12 \mathrm{H}-4,40$ & 104.89 & & 6.23 & & & & 0.29 & & & & \\
\hline $12 \mathrm{H}-4,102$ & 105.39 & 34.4 & 5.84 & & 1.71 & 65 & 0.19 & 9 & 313 & 275 & 422 \\
\hline $12 \mathrm{H}-5,24$ & 106.01 & 41.8 & 6.83 & & 1.81 & 65 & 0.20 & 9 & 343 & 300 & 415 \\
\hline $12 \mathrm{H}-5,40$ & 106.17 & 36.6 & 7.50 & 4.90 & 3.11 & 55 & 0.23 & 14 & 250 & & \\
\hline $12 \mathrm{H}-6,40$ & 107.35 & & 5.58 & & & & 0.18 & & & & \\
\hline $12 \mathrm{H}-6,82$ & 107.77 & 33.8 & 5.08 & & 1.02 & 50 & 0.24 & 4 & 218 & 311 & 415 \\
\hline $12 \mathrm{H}-6,120$ & 108.10 & 32.5 & 4.91 & & 1.01 & 55 & 0.16 & 6 & 245 & 275 & 419 \\
\hline $12 \mathrm{H}-7,40$ & 108.80 & 22.1 & 5.04 & & 2.39 & 60 & 0.20 & 12 & 305 & & 421 \\
\hline $12 \mathrm{H}-8,50$ & 110.04 & & 7.27 & & & & 0.21 & & & & \\
\hline $12 \mathrm{H}-8,59$ & 110.13 & 40.1 & 6.70 & & 1.89 & 60 & 0.21 & 9 & 280 & 264 & 421 \\
\hline $13 \mathrm{H}-1,42$ & 110.53 & & 7.37 & & & & 0.26 & & & & \\
\hline $12 \mathrm{H}-\mathrm{CC}, 20$ & 110.53 & 27.0 & 5.90 & & 2.66 & 50 & 0.34 & 8 & 235 & 190 & 418 \\
\hline $13 \mathrm{H}-1,59$ & 110.70 & 37.9 & 6.77 & & 2.22 & 60 & 0.15 & 15 & 300 & 260 & 413 \\
\hline $13 \mathrm{H}-2,40$ & 111.69 & 30.8 & 5.45 & 2.53 & 1.75 & & 0.15 & 12 & & & \\
\hline $13 \mathrm{H}-2,120$ & 112.49 & 32.9 & 6.66 & & 2.71 & 55 & 0.21 & 13 & 266 & 178 & 422 \\
\hline $13 \mathrm{H}-3,40$ & 112.97 & 29.8 & 7.46 & 5.53 & 3.88 & 60 & 0.30 & 13 & 308 & & 416 \\
\hline $13 \mathrm{H}-3,116$ & 113.73 & 26.8 & 4.84 & & 1.62 & 50 & 0.10 & 16 & 230 & 280 & 421 \\
\hline $13 \mathrm{H}-4,40$ & 114.24 & 36.9 & 6.77 & 3.71 & 2.34 & 70 & 0.25 & 9 & 362 & & 404 \\
\hline $13 \mathrm{H}-4,120$ & 115.04 & 23.2 & 4.49 & & 1.71 & 55 & 0.16 & 11 & 253 & 218 & 427 \\
\hline $13 \mathrm{H}-5,40$ & 115.52 & 27.8 & 6.20 & 3.96 & 2.86 & & 0.24 & 12 & & & \\
\hline $13 \mathrm{H}-5,120$ & 116.32 & 37.9 & 6.95 & & 2.40 & 60 & 0.26 & 9 & 300 & 241 & 411 \\
\hline $13 \mathrm{H}-6,42$ & 116.87 & & 7.83 & & & & 0.24 & & & & \\
\hline $13 \mathrm{H}-6,84$ & 117.29 & 41.2 & 6.99 & & 2.05 & 50 & 0.21 & 10 & 209 & 303 & 420 \\
\hline $13 \mathrm{H}-7,40$ & 118.07 & 27.9 & 6.21 & 3.97 & 2.86 & 55 & 0.29 & 10 & 269 & & \\
\hline $13 \mathrm{H}-7,120$ & 118.87 & 44.9 & 7.29 & & 1.90 & 55 & 0.21 & 9 & 266 & 278 & 421 \\
\hline $13 \mathrm{H}-9,42$ & 119.47 & & 7.08 & & & & 0.21 & & & & \\
\hline $14 \mathrm{H}-1,40$ & 119.87 & 22.8 & 3.98 & 1.61 & 1.24 & 45 & 0.11 & 11 & 190 & & 412 \\
\hline $14 \mathrm{H}-1,120$ & 120.67 & 17.9 & 3.12 & & 0.97 & 40 & 0.10 & 10 & 143 & 323 & 414 \\
\hline
\end{tabular}


Table 1 (continued).

\begin{tabular}{|c|c|c|c|c|c|c|c|c|c|c|c|}
\hline $\begin{array}{l}\text { Core, section, } \\
\text { interval }(\mathrm{cm})\end{array}$ & $\begin{array}{l}\text { Depth } \\
\text { (mbsf) }\end{array}$ & $\mathrm{CaCO}_{3}$ & $\mathrm{C}_{\text {tot }}$ & $\mathrm{C}_{\text {org' }}$ & $\mathrm{C}_{\text {org }}$ & MOM & $\mathrm{N}_{\text {tot }}$ & $\mathrm{C} / \mathrm{N}$ & $\mathrm{HI}$ & OI & $\mathrm{T}_{\max }$ \\
\hline $14 \mathrm{H}-1,135$ & 120.80 & 31.0 & 5.67 & & 1.95 & 65 & 0.26 & 8 & 319 & 222 & 412 \\
\hline $14 \mathrm{H}-2,40$ & 121.12 & 26.3 & 6.51 & 4.55 & 3.35 & 60 & 0.30 & 11 & 291 & & 415 \\
\hline $14 \mathrm{H}-2,94$ & 121.68 & 24.8 & 5.95 & & 2.97 & 65 & 0.30 & 10 & 336 & 200 & 408 \\
\hline $14 \mathrm{H}-2,108$ & 121.80 & 18.7 & 5.46 & & 3.22 & 65 & 0.27 & 12 & 355 & & 417 \\
\hline $14 \mathrm{H}-2,120$ & 121.89 & 24.7 & 5.63 & & 2.67 & 55 & 0.33 & 8 & 249 & 225 & 406 \\
\hline $14 \mathrm{H}-3,40$ & 122.49 & 21.8 & 5.11 & 3.19 & 2.49 & 55 & 0.24 & 10 & 278 & & 406 \\
\hline $14 \mathrm{H}-4,34$ & 123.63 & 33.4 & 5.94 & & 1.93 & & 0.24 & 8 & & & \\
\hline $14 \mathrm{H}-4,40$ & 123.69 & & 5.95 & & & & 0.28 & & & & \\
\hline $14 \mathrm{H}-5,34$ & 124.85 & 32.1 & 5.66 & & 1.81 & 45 & 0.18 & 10 & 197 & 266 & 425 \\
\hline $14 \mathrm{H}-5,53$ & 125.03 & 26.9 & 4.80 & 2.15 & 1.57 & 60 & 0.17 & 9 & 285 & & 418 \\
\hline $14 \mathrm{H}-6,40$ & 126.03 & & 3.88 & & & & 0.16 & & & & \\
\hline $14 \mathrm{H}-6,85$ & 126.48 & 25.6 & 5.66 & & 2.59 & 45 & 0.23 & 11 & 182 & 251 & 419 \\
\hline $14 \mathrm{H}-7,34$ & 127.29 & 26.7 & 5.54 & & 2.34 & 55 & 0.29 & 8 & 233 & 251 & 413 \\
\hline $14 \mathrm{H}-7,40$ & 127.35 & 23.2 & 5.60 & 3.67 & 2.82 & & 0.29 & 10 & & & \\
\hline $14 \mathrm{H}-8,40$ & 128.67 & & 6.28 & & & & 0.18 & & & & \\
\hline $14 \mathrm{H}-\mathrm{CC}, 17$ & 129.00 & 34.8 & 5.68 & & 1.50 & 60 & 0.15 & 10 & 281 & & 417 \\
\hline $15 \mathrm{H}-1,34$ & 129.49 & 24.4 & 4.61 & & 1.68 & 45 & 0.16 & 11 & 186 & 299 & 416 \\
\hline $15 \mathrm{H}-1,46$ & 129.61 & 15.8 & 4.15 & 2.68 & 2.26 & 45 & 0.17 & 13 & 162 & & \\
\hline $15 \mathrm{H}-2,37$ & 130.67 & & 5.03 & & & & 0.25 & & & & \\
\hline $15 \mathrm{H}-2,120$ & 131.49 & 26.0 & 5.86 & & 2.74 & 45 & 0.26 & 11 & 202 & 238 & 424 \\
\hline $15 \mathrm{H}-3,51$ & 132.06 & 20.5 & 5.97 & 4.42 & 3.52 & 65 & 0.30 & 12 & 328 & & 411 \\
\hline $15 \mathrm{H}-3,120$ & 132.76 & 29.2 & 5.69 & & 2.19 & 50 & 0.16 & 14 & 218 & 213 & 418 \\
\hline $15 \mathrm{H}-4,44$ & 133.32 & & 7.15 & & & & 0.24 & & & & \\
\hline $15 \mathrm{H}-4,88$ & 133.76 & 42.8 & 6.33 & & 1.19 & 50 & 0.13 & 9 & 222 & 408 & 417 \\
\hline $15 \mathrm{H}-5,43$ & 134.59 & & 6.87 & & & & 0.17 & & & & \\
\hline $15 \mathrm{H}-5,88$ & 135.03 & 34.7 & 6.03 & & 1.87 & 50 & 0.18 & 10 & 207 & 238 & 425 \\
\hline $15 \mathrm{H}-6,46$ & 135.88 & & 6.62 & & & & 0.24 & & & & \\
\hline $15 \mathrm{H}-6,84$ & 136.26 & 37.3 & 6.33 & & 1.85 & 55 & 0.24 & 8 & 242 & 259 & 417 \\
\hline $15 \mathrm{H}-7,34$ & 137.11 & 27.9 & 5.69 & & 2.34 & 60 & 0.31 & 8 & 282 & 305 & 415 \\
\hline $15 \mathrm{H}-7,46$ & 137.23 & 20.4 & 5.95 & 4.40 & 3.50 & & 0.30 & 12 & & & \\
\hline $15 \mathrm{H}-8,46$ & 138.60 & 19.2 & 4.99 & 3.33 & 2.69 & & 0.22 & 12 & & & \\
\hline $16 \mathrm{H}-1,92$ & 139.60 & 13.8 & 5.12 & & 3.47 & 70 & 0.32 & 11 & 371 & & 420 \\
\hline $16 \mathrm{H}-1,120$ & 139.84 & 17.2 & 5.15 & & 3.09 & 55 & 0.46 & 7 & 258 & 161 & 426 \\
\hline $16 \mathrm{H}-2,40$ & 140.47 & 27.3 & 6.01 & 3.76 & 2.73 & & 0.24 & 11 & & & \\
\hline $16 \mathrm{H}-2,120$ & 141.27 & 22.3 & 5.85 & & 3.17 & 55 & 0.29 & 11 & 262 & 214 & 411 \\
\hline $16 \mathrm{H}-3,40$ & 141.89 & 21.6 & 5.63 & 3.88 & 3.04 & 70 & 0.27 & 11 & 381 & & 414 \\
\hline $16 \mathrm{H}-3,120$ & 142.69 & 35.0 & 5.71 & & 1.51 & 55 & 0.13 & 12 & 260 & 345 & 413 \\
\hline $16 \mathrm{H}-4,20$ & 143.12 & 34.6 & 5.88 & & 1.73 & 65 & 0.17 & 10 & 315 & & 420 \\
\hline $16 \mathrm{H}-4,40$ & 143.32 & 28.8 & 6.39 & 4.12 & 2.93 & & 0.26 & 11 & & & \\
\hline $16 \mathrm{H}-4,120$ & 144.12 & 23.6 & 5.27 & & 2.44 & 50 & 0.23 & 11 & 211 & 185 & 422 \\
\hline $16 \mathrm{H}-5,40$ & 144.74 & 27.6 & 6.36 & 4.21 & 3.05 & 50 & 0.27 & 11 & 216 & & 414 \\
\hline $16 \mathrm{H}-5,120$ & 145.54 & 36.1 & 6.82 & & 2.49 & 60 & 0.20 & 12 & 280 & 240 & 415 \\
\hline $16 \mathrm{H}-6,40$ & 146.17 & & 4.62 & & & & 0.15 & & & & \\
\hline $16 \mathrm{H}-6,120$ & 146.97 & 36.0 & 5.63 & & 1.31 & 65 & 0.13 & 10 & 346 & 229 & 410 \\
\hline $16 \mathrm{H}-7,40$ & 147.67 & 27.3 & 5.66 & 3.28 & 2.38 & 55 & 0.20 & 12 & 240 & & 412 \\
\hline $17 \mathrm{H}-1,40$ & 148.60 & 32.9 & 7.64 & 5.50 & 3.69 & 55 & 0.30 & 12 & 243 & & 407 \\
\hline $17 \mathrm{H}-1,120$ & 149.40 & 30.7 & 6.73 & & 3.05 & 65 & 0.29 & 11 & 322 & 229 & 416 \\
\hline $17 \mathrm{H}-2,34$ & 150.04 & 31.2 & 6.64 & & 2.90 & 60 & 0.29 & 10 & 298 & 187 & 425 \\
\hline $17 \mathrm{H}-2,40$ & 150.10 & & 6.61 & & & & 0.28 & & & & \\
\hline $17 \mathrm{H}-3,40$ & 151.60 & 42.0 & 7.87 & 4.88 & 2.83 & 65 & 0.36 & 8 & 318 & & 417 \\
\hline $17 \mathrm{H}-3,120$ & 152.40 & 27.1 & 6.36 & & 3.11 & 55 & 0.33 & 9 & 262 & 216 & 422 \\
\hline $17 \mathrm{H}-4,40$ & 153.10 & & 6.17 & & & & 0.24 & & & & \\
\hline $17 \mathrm{H}-4,120$ & 153.90 & 16.2 & 4.21 & & 2.27 & 60 & 0.27 & 8 & 281 & 188 & 425 \\
\hline $17 \mathrm{H}-5,34$ & 154.54 & 31.3 & 6.01 & & 2.25 & 55 & 0.26 & 9 & 247 & 220 & 420 \\
\hline $17 \mathrm{H}-5,40$ & 154.60 & 26.0 & 5.97 & 3.85 & 2.85 & 55 & 0.27 & 11 & 268 & & 410 \\
\hline $17 \mathrm{H}-6,34$ & 156.04 & 32.2 & 5.83 & & 1.97 & 60 & 0.12 & 16 & 293 & 213 & 425 \\
\hline $17 \mathrm{H}-6,46$ & 156.16 & 32.9 & 6.48 & 3.77 & 2.53 & 55 & 0.22 & 11 & 262 & & 409 \\
\hline $19 X-1,44$ & 158.19 & 47.3 & 7.49 & 3.44 & 1.81 & 50 & 0.17 & 11 & 235 & & 419 \\
\hline $19 X-1,84$ & 158.59 & 24.4 & 3.96 & & 1.03 & 45 & 0.11 & 9 & 170 & 403 & 409 \\
\hline $19 X-2,34$ & 159.42 & 24.5 & 5.14 & & 2.20 & 50 & 0.23 & 10 & 231 & 228 & 410 \\
\hline $19 X-2,40$ & 159.48 & 21.6 & 4.63 & 2.60 & 2.04 & & 0.21 & 10 & & & \\
\hline $19 X-3,84$ & 160.88 & 29.7 & 6.44 & & 2.88 & 55 & 0.28 & 10 & 247 & 217 & 425 \\
\hline $19 X-4,35$ & 161.76 & 21.5 & 6.33 & 4.78 & 3.75 & 55 & 0.29 & 13 & 251 & & 414 \\
\hline $19 X-4,120$ & 162.61 & 31.6 & 6.34 & & 2.55 & 55 & 0.25 & 10 & 256 & 205 & 423 \\
\hline $19 X-5,44$ & 162.96 & 28.5 & 6.38 & 4.14 & 2.96 & 50 & 0.24 & 12 & 224 & & \\
\hline $19 X-5,84$ & 163.36 & & & & & & & & & & \\
\hline $19 X-6,34$ & 164.38 & 23.1 & 5.40 & & 2.63 & 50 & 0.22 & 12 & 228 & 194 & 420 \\
\hline $19 X-6,44$ & 164.48 & 16.4 & 5.49 & 4.21 & 3.52 & 55 & 0.34 & 10 & 253 & & 414 \\
\hline $19 X-7,40$ & 165.54 & 21.2 & 5.44 & 3.68 & 2.90 & 55 & 0.29 & 10 & 253 & & 415 \\
\hline $19 X-7,120$ & 166.34 & 39.3 & 6.14 & & 1.42 & 50 & 0.18 & 8 & 221 & 341 & 425 \\
\hline $20 X-1,40$ & 167.35 & & 5.44 & & & & 0.15 & & & & \\
\hline $20 X-1,120$ & 168.15 & 22.9 & 3.86 & & 1.11 & 45 & 0.16 & 7 & 203 & 430 & 408 \\
\hline $20 \mathrm{X}-2,38$ & 168.51 & 30.9 & 5.43 & 2.49 & 1.72 & 45 & 0.21 & 8 & 177 & & 419 \\
\hline $20 X-2,84$ & 168.97 & 42.2 & 6.35 & & 1.29 & 45 & 0.17 & 8 & 191 & 424 & 415 \\
\hline $20 X-3,34$ & 169.50 & 47.0 & 6.74 & & 1.10 & 55 & 0.15 & 7 & 279 & 473 & 418 \\
\hline $20 X-3,40$ & 169.56 & 43.1 & 7.04 & 3.28 & 1.87 & 45 & 0.13 & 14 & 180 & & 422 \\
\hline $20 \times-7,40$ & 173.14 & 9.5 & 3.67 & 2.80 & 2.54 & & 0.12 & 21 & & & \\
\hline
\end{tabular}


Table 1 (continued).

\begin{tabular}{|c|c|c|c|c|c|c|c|c|c|c|c|}
\hline $\begin{array}{l}\text { Core, section, } \\
\text { interval }(\mathrm{cm})\end{array}$ & $\begin{array}{l}\text { Depth } \\
\text { (mbsf) }\end{array}$ & $\mathrm{CaCO}_{3}$ & $C_{\text {tot }}$ & $\mathrm{C}_{\text {org' }^{\prime}}$ & $\mathrm{C}_{\text {org }}$ & MOM & $\mathrm{N}_{\text {tot }}$ & $\mathrm{C} / \mathrm{N}$ & HI & OI & $\mathrm{T}_{\max }$ \\
\hline $20 X-7,84$ & 173.58 & 32.3 & 5.07 & & 1.19 & 50 & 0.11 & 11 & 212 & 400 & 414 \\
\hline $20 \mathrm{X}-8,38$ & 173.92 & 49.1 & 8.19 & 4.52 & 2.30 & 50 & 0.21 & 11 & 208 & & 418 \\
\hline $20 \mathrm{X}-8,120$ & 174.74 & 44.5 & 7.60 & & 2.26 & 60 & 0.24 & 9 & 296 & 349 & 416 \\
\hline $20 \mathrm{X}-9,34$ & 174.96 & 35.4 & 7.85 & 5.58 & 3.61 & 55 & 0.31 & 12 & 246 & & 416 \\
\hline $20 X-9,84$ & 175.46 & 38.2 & 7.62 & & 3.04 & 55 & 0.31 & 10 & 242 & 241 & 418 \\
\hline $21 X-1,40$ & 176.90 & 31.2 & 6.23 & 3.61 & 2.48 & 50 & 0.22 & 11 & 208 & & 418 \\
\hline $21 X-2,37$ & 178.34 & & 7.33 & & & & 0.25 & & & & \\
\hline $21 X-2,84$ & 178.81 & 40.5 & 6.74 & & 1.88 & 50 & 0.10 & 19 & 231 & 247 & 422 \\
\hline $21 X-3,41$ & 179.88 & 37.9 & 6.55 & 3.22 & 2.00 & 50 & 0.19 & 11 & 226 & & 417 \\
\hline $21 X-4,39$ & 180.76 & 36.2 & 6.14 & 2.81 & 1.79 & 45 & 0.21 & 9 & 190 & & 419 \\
\hline $21 X-4,110$ & 181.46 & 42.6 & 6.12 & & 1.01 & 45 & 0.06 & 17 & 192 & 462 & 411 \\
\hline $21 X-5,38$ & 181.96 & 26.0 & 5.13 & 2.72 & 2.01 & 50 & 0.22 & 9 & 224 & & 419 \\
\hline $21 X-5,120$ & 182.76 & 38.4 & 6.22 & & 1.61 & 65 & 0.19 & 8 & 352 & 316 & 411 \\
\hline $21 X-6,10$ & 183.00 & 38.5 & 6.20 & & 1.58 & 65 & & & 316 & & 419 \\
\hline $21 X-6,40$ & 183.31 & 40.5 & 6.60 & 2.92 & 1.74 & & 0.20 & 9 & & & \\
\hline $21 X-6,54$ & 183.45 & 43.8 & 6.64 & & 1.38 & 50 & 0.19 & 7 & 213 & 406 & 410 \\
\hline $21 X-8,40$ & 185.91 & 13.8 & 4.01 & 2.73 & 2.35 & 45 & 0.25 & 9 & 200 & & 417 \\
\hline $22 \mathrm{X}-2,40$ & 188.04 & 14.7 & 5.17 & 4.00 & 3.41 & 60 & 0.34 & 10 & 283 & & \\
\hline $22 X-2,71$ & 188.34 & 19.9 & 5.20 & & 2.81 & 55 & 0.28 & 10 & 243 & 210 & 409 \\
\hline $22 X-3,46$ & 189.40 & 21.8 & 6.14 & 4.51 & 3.53 & 55 & 0.32 & 11 & 244 & & 417 \\
\hline $22 X-4,38$ & 190.69 & 29.3 & 6.30 & 3.94 & 2.79 & 55 & 0.38 & 10 & 256 & & 418 \\
\hline $22 X-5,45$ & 192.00 & 32.3 & 6.78 & 4.29 & 2.90 & 55 & 0.27 & 11 & 274 & & 421 \\
\hline $22 \mathrm{X}-6,55$ & 193.29 & 33.3 & 6.52 & 3.79 & 2.53 & 55 & 0.28 & 9 & 272 & & 409 \\
\hline $22 X-7,47$ & 194.63 & 37.5 & 6.0 & 3.84 & 2.40 & 55 & 0.20 & 12 & 264 & & 409 \\
\hline $22 \mathrm{X}-7,100$ & 195.16 & 40.9 & 6.96 & & 2.05 & 55 & 0.20 & 10 & 267 & 278 & 414 \\
\hline $23 \mathrm{X}-1,40$ & 196.08 & & 7.38 & & & & 0.22 & & & & \\
\hline $23 \mathrm{X}-1,119$ & 196.80 & 47.7 & 7.66 & & 1.94 & 65 & 0.23 & 8 & 332 & & 422 \\
\hline $23 \mathrm{X}-1,123$ & 196.90 & 21.9 & 4.33 & & 1.70 & 70 & 0.18 & 9 & 392 & 291 & 400 \\
\hline $23 \mathrm{X}-1,126$ & 196.92 & 49.1 & 7.50 & & 1.61 & 55 & 0.20 & 8 & 257 & 370 & 414 \\
\hline $23 \mathrm{X}-2,48$ & 197.39 & 43.3 & 7.79 & 4.57 & 2.59 & 55 & 0.20 & 13 & 236 & & 409 \\
\hline $23 \mathrm{X}-2,108$ & 197.99 & 46.4 & 7.56 & & 1.99 & 70 & 0.19 & 10 & 362 & 212 & 408 \\
\hline $23 \mathrm{X}-3,40$ & 198.60 & 45.4 & 8.05 & 4.77 & 2.61 & 50 & 0.21 & 12 & 218 & & 408 \\
\hline $23 \mathrm{X}-3,56$ & 198.78 & 48.3 & 7.58 & & 1.78 & 55 & 0.20 & 9 & 251 & 335 & 413 \\
\hline $23 \mathrm{X}-4,40$ & 199.70 & 40.2 & 7.95 & 5.23 & 3.13 & 50 & 0.26 & 12 & 214 & & 417 \\
\hline $23 \mathrm{X}-4,126$ & 200.56 & 51.1 & 8.24 & & 2.11 & 55 & 0.20 & 11 & 277 & 304 & 408 \\
\hline $23 \mathrm{X}-5,35$ & 201.04 & 47.1 & 7.76 & & 2.11 & 55 & 0.24 & 9 & 263 & 300 & 411 \\
\hline $23 X-5,40$ & 201.09 & & 7.87 & & & & 0.25 & & & & \\
\hline $23 X-6,64$ & 202.49 & 39.9 & 6.90 & & 2.11 & 50 & 0.17 & 12 & 229 & 276 & 408 \\
\hline $23 \times-7,18$ & 203.30 & 24.0 & 7.12 & 5.58 & 4.24 & 50 & 0.34 & 12 & 224 & & 413 \\
\hline $23 X-7,34$ & 203.46 & 34.2 & 7.06 & & 2.96 & 55 & 0.23 & 13 & 280 & 243 & 413 \\
\hline $23 X-8,76$ & 205.18 & 40.0 & 6.91 & & 2.11 & 55 & 0.19 & 11 & 245 & 264 & 409 \\
\hline $23 X-9,52$ & 205.42 & 36.0 & 6.89 & 4.02 & 2.57 & & 0.17 & 15 & & & \\
\hline $23 \mathrm{X}-9,90$ & 205.50 & 41.8 & 7.22 & & 2.21 & 65 & 0.22 & 10 & 353 & & 420 \\
\hline $24 \mathrm{X}-1,14$ & 205.52 & 44.0 & 6.93 & & 1.65 & 60 & 0.16 & 10 & 298 & 312 & 407 \\
\hline $24 X-2,63$ & 207.19 & 44.4 & 7.45 & 3.82 & 2.12 & 55 & 0.25 & 8 & 273 & & 413 \\
\hline $24 \mathrm{X}-2,130$ & 207.89 & 36.9 & 6.71 & & 2.28 & 55 & 0.27 & 8 & 244 & 295 & 412 \\
\hline $24 \mathrm{X}-3,40$ & 208.36 & 30.1 & 7.23 & 5.18 & 3.62 & 55 & 0.30 & 12 & 237 & & 419 \\
\hline $24 X-3,76$ & 208.74 & 45.5 & 7.69 & & 2.23 & 45 & 0.24 & 9 & 203 & 277 & 414 \\
\hline $24 X-4,17$ & 209.40 & 36.7 & 8.14 & 5.90 & 3.73 & 50 & 0.28 & 13 & 234 & & 416 \\
\hline $24 \mathrm{X}-4,113$ & 210.40 & 41.9 & 6.99 & & 1.96 & 55 & 0.26 & 8 & 244 & 242 & 415 \\
\hline $24 \mathrm{X}-5,18$ & 210.85 & & 5.13 & & & & 0.21 & & & & \\
\hline $24 X-5,38$ & 211.05 & 14.9 & 5.09 & 3.88 & 3.30 & 55 & 0.25 & 13 & 273 & & 418 \\
\hline $24 X-5,58$ & 211.25 & 22.7 & 4.81 & & 2.09 & 45 & 0.30 & 7 & 206 & 285 & 422 \\
\hline $24 X-6,44$ & 212.40 & 27.6 & 7.40 & 5.65 & 4.09 & & 0.28 & 15 & & & \\
\hline $24 \mathrm{X}-6,88$ & 212.80 & 30.5 & 6.88 & & 3.22 & 70 & 0.32 & 10 & 375 & & 422 \\
\hline $24 X-6,100$ & 212.96 & 30.4 & 6.68 & & 3.03 & 50 & 0.30 & 10 & 237 & 255 & 407 \\
\hline $24 \mathrm{X}-7,33$ & 213.68 & 35.5 & 6.45 & 3.39 & 2.19 & 55 & 0.18 & 12 & 256 & & 421 \\
\hline $24 X-7,46$ & 213.81 & 41.3 & 6.97 & & 2.01 & 50 & 0.24 & 8 & 221 & 345 & 412 \\
\hline $24 X-8,26$ & 214.78 & 32.8 & 6.82 & 4.29 & 2.88 & & 0.28 & 10 & & & \\
\hline $24 \mathrm{X}-8,17$ & 214.88 & 36.8 & 6.24 & & 1.82 & 65 & 0.23 & 8 & 310 & 287 & 417 \\
\hline $24 X-9,8$ & 215.00 & 38.0 & 6.69 & & 2.13 & 50 & 0.29 & 7 & 221 & 276 & 406 \\
\hline $25 X-1,41$ & 215.30 & 34.9 & 6.57 & 3.66 & 2.38 & 50 & 0.22 & 11 & 230 & & 419 \\
\hline $25 \mathrm{X}-1,118$ & 216.03 & 42.0 & 6.90 & & 1.86 & 70 & 0.24 & 8 & 378 & 245 & 426 \\
\hline $25 \mathrm{X}-1,123$ & 216.08 & 36.5 & 7.17 & 4.40 & 2.80 & 55 & 0.24 & 12 & 245 & & 415 \\
\hline $25 \mathrm{X}-1,126$ & 216.11 & 41.5 & 7.24 & & 2.26 & 55 & 0.13 & 17 & 245 & 225 & 419 \\
\hline $25 \mathrm{X}-2,38$ & 216.70 & 33.0 & 7.60 & 5.43 & 3.64 & 50 & 0.28 & 13 & 214 & & \\
\hline $25 \mathrm{X}-2,120$ & 217.50 & 46.0 & 6.85 & & 1.33 & 75 & 0.24 & 6 & 511 & 508 & 415 \\
\hline $25 \mathrm{X}-3,40$ & 218.15 & 39.0 & 6.97 & 3.75 & 2.29 & 55 & 0.19 & 12 & 254 & & 415 \\
\hline $25 \mathrm{X}-3,69$ & 218.45 & 40.9 & 6.83 & & 1.92 & 50 & 0.23 & 8 & 218 & 298 & 414 \\
\hline $25 \mathrm{X}-4,40$ & 219.61 & 29.1 & 5.29 & 2.54 & 1.80 & 65 & 0.22 & 8 & 322 & & 419 \\
\hline $25 \mathrm{X}-4,49$ & 219.70 & 28.2 & 5.10 & & 1.72 & 50 & 0.17 & 10 & 232 & 271 & 408 \\
\hline $25 \mathrm{X}-5,38$ & 221.02 & 25.3 & 5.34 & 3.09 & 2.31 & & 0.45 & 5 & & & \\
\hline $25 X-5,65$ & 221.29 & 37.6 & 6.26 & & 1.75 & 50 & 0.24 & 7 & 261 & 258 & 420 \\
\hline $25 \mathrm{X}-5,121$ & 221.80 & 42.8 & 7.04 & 3.33 & 1.90 & 50 & 0.19 & 10 & 221 & & 420 \\
\hline $25 X-6,16$ & 222.25 & & 6.94 & & & & 0.15 & & & & \\
\hline $25 \mathrm{X}-6,41$ & 222.51 & 48.5 & 7.19 & & 1.37 & 50 & 0.16 & 9 & 234 & 395 & 412 \\
\hline $25 \mathrm{X}-6,64$ & 222.73 & 48.0 & 7.15 & & 1.39 & 90 & 0.13 & 11 & 879 & 465 & 416 \\
\hline
\end{tabular}


Table 1 (continued).

\begin{tabular}{|c|c|c|c|c|c|c|c|c|c|c|c|}
\hline $\begin{array}{l}\text { Core, section, } \\
\text { interval }(\mathrm{cm})\end{array}$ & $\begin{array}{l}\text { Depth } \\
\text { (mbsf) }\end{array}$ & $\mathrm{CaCO}_{3}$ & $\mathrm{C}_{\text {tot }}$ & $\mathrm{C}_{\text {org' }}$ & $\mathrm{C}_{\text {org }}$ & MOM & $\mathrm{N}_{\text {tot }}$ & $\mathrm{C} / \mathrm{N}$ & HI & OI & $\mathrm{T}_{\max }$ \\
\hline $25 X-7,34$ & 223.88 & 44.8 & 7.19 & & 1.81 & 50 & 0.17 & 11 & 236 & 328 & 404 \\
\hline $25 X-7,60$ & 224.14 & 31.6 & 6.49 & 3.95 & 2.70 & 50 & 0.30 & 9 & 212 & & \\
\hline $26 \mathrm{X}-1,21$ & 224.56 & 42.4 & 6.53 & & 1.44 & 40 & 0.14 & 10 & 155 & 415 & 422 \\
\hline $26 X-1,41$ & 224.76 & & 6.88 & & & & 0.20 & & & & \\
\hline $26 \mathrm{X}-2,30$ & 225.71 & & & & 1.31 & 60 & & 12 & 297 & & \\
\hline $26 \mathrm{X}-2,49$ & 225.86 & 39.3 & 6.82 & 3.46 & 2.10 & 50 & 0.23 & 9 & 210 & & 424 \\
\hline $26 \mathrm{X}-2,91$ & 226.26 & 49.9 & 7.11 & & 1.12 & 50 & 0.12 & 9 & 209 & 417 & 427 \\
\hline $26 X-2,96$ & 226.30 & 49.2 & 7.17 & & 1.27 & 60 & 0.25 & 5 & 282 & 365 & 420 \\
\hline $26 X-3,76$ & 227.28 & 35.4 & 5.95 & 2.64 & 1.71 & 45 & 0.22 & 8 & 180 & & 421 \\
\hline $26 X-3,84$ & 227.36 & 38.6 & 5.95 & & 1.32 & 45 & 0.20 & 7 & 194 & 346 & 419 \\
\hline $26 X-4,47$ & 228.15 & 32.9 & 6.14 & 3.26 & 2.19 & 50 & 0.21 & 10 & 214 & & \\
\hline $26 X-4,69$ & 228.35 & 34.0 & 6.03 & & 1.95 & 65 & 0.25 & 8 & 314 & & 423 \\
\hline $26 X-4,84$ & 228.52 & 30.9 & 5.38 & & 1.67 & 55 & 0.22 & 8 & 242 & 258 & 425 \\
\hline $26 X-4,86$ & 228.53 & 32.0 & 5.59 & & 1.75 & 60 & 0.35 & 5 & 308 & 271 & 415 \\
\hline $26 x-5,5$ & 229.06 & 28.5 & 5.17 & & 1.75 & 45 & 0.19 & 9 & 170 & 222 & 425 \\
\hline $26 \mathrm{X}-6,30$ & 230.41 & 39.4 & 6.72 & & 1.99 & 50 & 0.15 & 13 & 230 & 228 & 425 \\
\hline $26 X-6,39$ & 230.50 & 34.0 & 7.08 & 4.55 & 3.00 & 55 & 0.26 & 12 & 253 & & 419 \\
\hline $26 \times-7,30$ & 231.57 & 42.0 & 6.40 & & 1.36 & 40 & 0.19 & 7 & 166 & 342 & 425 \\
\hline $26 \times-7,34$ & 231.61 & 37.4 & 6.31 & 2.91 & 1.82 & 50 & 0.21 & 9 & 218 & & 422 \\
\hline $26 X-8,30$ & 232.73 & 9.4 & 2.83 & & 1.70 & 35 & 0.16 & 11 & 105 & 284 & 418 \\
\hline $26 \mathrm{X}-8,36$ & 232.79 & 18.1 & 3.56 & 1.69 & 1.38 & 45 & 0.21 & 7 & 189 & & 416 \\
\hline $26 \times-9,10$ & 233.73 & 11.4 & 2.96 & & 1.59 & 40 & 0.23 & 7 & 135 & 274 & 416 \\
\hline $26 X-9,43$ & 234.04 & & 3.76 & & & & 0.23 & & & & \\
\hline $27 X-1,40$ & 234.30 & 43.2 & 6.91 & 3.04 & 1.73 & 40 & 0.16 & 11 & 163 & & \\
\hline $27 X-1,68$ & 234.57 & 48.9 & 6.97 & & 1.10 & 50 & 0.14 & 8 & 206 & 421 & 424 \\
\hline $27 X-2,48$ & 235.85 & 47.9 & 7.36 & 3.09 & 1.61 & 45 & 0.19 & 8 & 188 & & 423 \\
\hline $27 X-2,102$ & 236.37 & 49.3 & 7.21 & & 1.29 & 65 & 0.14 & 9 & 312 & 323 & 423 \\
\hline $27 X-2,103$ & 236.38 & 49.1 & 7.28 & & 1.39 & 60 & 0.14 & 10 & 284 & 306 & 421 \\
\hline $27 X-3,60$ & 237.42 & 49.4 & 7.74 & 3.59 & 1.82 & 50 & 0.19 & 10 & 235 & & 420 \\
\hline $27 X-3,120$ & 238.02 & 44.8 & 7.44 & & 2.06 & 50 & 0.13 & 16 & 236 & 226 & 428 \\
\hline $27 X-4,41$ & 238.70 & 39.5 & 7.40 & 4.40 & 2.66 & 50 & 0.21 & 13 & 236 & & \\
\hline $27 X-4,86$ & 239.15 & 42.6 & 7.07 & & 1.96 & 50 & 0.21 & 9 & 236 & 291 & 417 \\
\hline $27 X-5,18$ & 239.97 & 28.7 & 6.47 & 4.25 & 3.03 & 50 & 0.30 & 10 & 241 & & \\
\hline $27 X-5,100$ & 240.76 & 26.2 & 6.35 & & 3.21 & 65 & 0.29 & 11 & 326 & 195 & 422 \\
\hline $27 X-5,120$ & 240.96 & 21.7 & 5.79 & & 3.19 & 50 & 0.25 & 13 & 220 & 176 & 424 \\
\hline $27 X-6,26$ & 241.52 & & 6.17 & & & & 0.26 & & & & \\
\hline $27 X-6,68$ & 241.92 & 41.9 & 6.37 & & 1.34 & 45 & 0.06 & 22 & 184 & 299 & 430 \\
\hline $27 X-7,48$ & 243.20 & 43.2 & 7.22 & 3.58 & 2.03 & 50 & 0.20 & 10 & 228 & & 428 \\
\hline $27 X-7,62$ & 243.33 & 28.9 & 5.70 & & 2.23 & 60 & 0.18 & 12 & 301 & 240 & 422 \\
\hline $28 \mathrm{X}-1,40$ & 243.72 & 12.5 & 5.03 & 4.03 & 3.52 & 50 & 0.34 & 10 & 211 & & 412 \\
\hline $28 \mathrm{X}-1,120$ & 244.52 & 10.8 & 3.75 & & 2.45 & 55 & 0.23 & 11 & 256 & 198 & 418 \\
\hline $28 X-2,47$ & 245.20 & & 5.35 & & & & 0.34 & & & & \\
\hline $28 X-2,120$ & 245.92 & 29.1 & 5.91 & & 2.42 & 50 & 0.29 & 8 & 230 & 297 & 407 \\
\hline $28 \mathrm{X}-3,37$ & 246.47 & 25.7 & 6.52 & 4.62 & 3.43 & 50 & 0.26 & 13 & 216 & & 419 \\
\hline $28 \mathrm{X}-3,115$ & 247.27 & 34.2 & 6.82 & & 2.72 & 55 & 0.28 & 10 & 273 & 231 & 414 \\
\hline $28 X-4,043$ & 247.93 & 23.9 & 5.64 & 3.64 & 2.77 & 45 & 0.28 & 10 & 205 & & 423 \\
\hline $28 \mathrm{X}-4,120$ & 248.72 & 14.0 & 5.00 & & 3.32 & 50 & 0.38 & 9 & 243 & 173 & 429 \\
\hline $28 X-5,37$ & 249.30 & 2.2 & 4.01 & 3.83 & 3.75 & 50 & 0.32 & 12 & 238 & & 415 \\
\hline $28 X-5,110$ & 250.00 & 16.3 & 5.39 & & 3.44 & 70 & 0.36 & 10 & 379 & & 421 \\
\hline $28 \mathrm{X}-5,118$ & 250.10 & 19.3 & 5.50 & & 3.18 & 55 & 0.31 & 10 & 251 & 194 & 422 \\
\hline $28 \mathrm{X}-6,28$ & 250.61 & & 6.53 & & & & 0.34 & & & & \\
\hline $28 X-6,92$ & 251.25 & 33.1 & 6.67 & & 2.70 & 55 & 0.12 & 23 & 271 & 174 & 425 \\
\hline $28 \times-7,30$ & 252.08 & 35.1 & 6.72 & & 2.51 & 55 & 0.27 & 9 & 246 & 225 & 421 \\
\hline $28 \times-7,37$ & 252.15 & 27.5 & 6.79 & 4.81 & 3.49 & 50 & 0.27 & 13 & 215 & & 414 \\
\hline $29 X-2,32$ & 254.62 & 7.3 & 4.11 & 3.49 & 3.24 & 50 & 0.28 & 12 & 232 & & 427 \\
\hline $29 X-2,97$ & 255.22 & 14.2 & 3.96 & & 2.26 & 55 & 0.24 & 9 & 274 & 214 & 416 \\
\hline $29 \mathrm{X}-2,124$ & 255.47 & 10.3 & 3.22 & & 1.98 & 45 & 0.26 & 8 & 182 & 221 & 431 \\
\hline $29 X-3,38$ & 256.12 & 7.4 & 3.62 & 2.95 & 2.73 & 50 & 0.29 & 9 & 221 & & 413 \\
\hline $29 X-4,39$ & 257.47 & 33.4 & 5.98 & 2.96 & 1.97 & 45 & 0.22 & 9 & 200 & & 417 \\
\hline $29 X-4,101$ & 258.07 & 37.3 & 6.12 & & 1.64 & 60 & 0.21 & 8 & 293 & 323 & 412 \\
\hline $29 X-5,40$ & 258.90 & & 5.86 & & & & 0.25 & & & & \\
\hline $29 X-5,56$ & 259.05 & 24.3 & 4.78 & & 1.86 & 50 & 0.19 & 10 & 231 & 255 & 420 \\
\hline $29 X-6,39$ & 260.30 & & 6.53 & & & & 0.21 & & & & \\
\hline $29 X-6,56$ & 260.46 & 42.6 & 6.26 & & 1.15 & 60 & 0.07 & 16 & 228 & 390 & 417 \\
\hline $29 X-7,42$ & 261.84 & & 5.23 & & & & 0.22 & & & & \\
\hline $29 X-7,44$ & 261.86 & 6.8 & 4.17 & & 3.35 & 35 & 0.13 & 26 & 99 & 122 & 431 \\
\hline $29 X-8,26$ & 262.15 & 8.8 & 3.32 & & 2.26 & 60 & 0.22 & 10 & 308 & & 423 \\
\hline $29 X-8,38$ & 262.24 & & 3.26 & & & & 0.28 & & & & \\
\hline $29 X-8,44$ & 262.30 & 6.8 & 3.50 & & 2.68 & 60 & 0.24 & 11 & 294 & 127 & 427 \\
\hline $30 \times-1,44$ & 262.86 & 3.3 & 2.84 & 2.53 & 2.45 & 70 & 0.23 & 10 & 416 & & \\
\hline $30 X-1,50$ & 262.90 & 10.5 & 3.02 & & 1.76 & 50 & 0.20 & 9 & 214 & 238 & 428 \\
\hline $30 X-3,45$ & 265.85 & 19.8 & 4.56 & 2.72 & 2.18 & & 0.20 & 11 & & & \\
\hline $30 X-3,54$ & 265.94 & 17.6 & 4.17 & & 2.06 & 55 & 0.22 & 9 & 253 & 224 & 424 \\
\hline $30 X-4,40$ & 267.30 & & 5.36 & & & & 0.29 & & & & \\
\hline $30 \times-4,49$ & 267.39 & 28.8 & 5.82 & & 2.36 & 55 & 0.28 & 8 & 272 & 162 & 0 \\
\hline $30 X-5,48$ & 268.89 & 25.5 & 5.48 & 3.25 & 2.42 & 50 & 0.25 & 10 & 235 & & 418 \\
\hline $30 \times-5,119$ & 269.59 & 22.5 & 5.64 & & 2.94 & 60 & 0.27 & 11 & 294 & 195 & 0 \\
\hline $30 X-6,87$ & 270.77 & 27.3 & 6.46 & 4.38 & 3.18 & 55 & 0.27 & 12 & 239 & & 414 \\
\hline
\end{tabular}


Table 1 (continued).

\begin{tabular}{llllllllllll}
\hline $\begin{array}{c}\text { Core, section, } \\
\text { interval (cm) }\end{array}$ & $\begin{array}{l}\text { Depth } \\
\text { (mbsf) }\end{array}$ & $\mathrm{CaCO}_{3}$ & $\mathrm{C}_{\text {tot }}$ & $\mathrm{C}_{\text {org }}$ & $\mathrm{C}_{\text {org }}$ & $\mathrm{MOM}$ & $\mathrm{N}_{\text {tot }}$ & $\mathrm{C} / \mathrm{N}$ & $\mathrm{HI}$ & OI & $\mathrm{T}_{\text {max }}$ \\
\hline $31 \mathrm{X}-1,40$ & 272.29 & & 6.16 & & & & 0.36 & & & & \\
$31 \mathrm{X}-1,119$ & 273.09 & 30.4 & 5.95 & & 2.30 & 55 & 0.22 & 10 & 245 & 248 & 418 \\
$31 \mathrm{X}-2,40$ & 273.80 & & 5.77 & & & & 0.29 & & & & \\
$31 \mathrm{X}-2,121$ & 274.61 & 34.2 & 5.93 & & 1.83 & 50 & 0.25 & 7 & 219 & 274 & 428 \\
$31 \mathrm{X}-3,40$ & 275.30 & 18.8 & 5.77 & 4.33 & 3.52 & 55 & 0.33 & 11 & 261 & & 413 \\
$31 \mathrm{X}-3,54$ & 275.44 & 21.6 & 5.87 & & 3.28 & 60 & 0.22 & 15 & 282 & 140 & 427 \\
$32 \mathrm{X}-1,47$ & 281.07 & 9.8 & 3.69 & 2.78 & 2.51 & & 0.21 & 12 & & & \\
$32 \mathrm{X}-1,80$ & 281.40 & 45.2 & 6.25 & & 0.83 & 65 & 0.08 & 10 & 310 & 460 & 409 \\
$33 \mathrm{X}-1,40$ & 291.30 & 9.8 & 3.62 & 2.71 & 2.44 & 50 & 0.21 & 12 & 218 & & 423 \\
$33 \mathrm{X}-1,120$ & 292.10 & 27.9 & 4.80 & & 1.45 & 45 & 0.20 & 7 & 185 & 348 & 421 \\
$33 \mathrm{X}-2,40$ & 292.80 & & 4.93 & & & & 0.24 & & & & \\
$33 \mathrm{X}-2,109$ & 293.49 & 32.8 & 5.15 & & 1.21 & 45 & 0.10 & 12 & 192 & 402 & 423 \\
$33 \mathrm{X}-3,43$ & 294.33 & 22.4 & 4.90 & 2.85 & 2.21 & 60 & 0.24 & 9 & 296 & & 418 \\
$33 \mathrm{X}-3,77$ & 294.67 & 11.5 & 3.12 & & 1.74 & 40 & 0.16 & 11 & 152 & 195 & 431 \\
$33 \mathrm{X}-3,135$ & 295.35 & 6.0 & 2.95 & & 2.23 & 70 & & & 378 & & 427 \\
$33 \mathrm{X}-4,43$ & 295.83 & 20.0 & 4.49 & 2.61 & 2.09 & & 0.27 & 8 & & & \\
$33 \mathrm{X}-4,50$ & 295.90 & 23.9 & 4.49 & & 1.62 & 45 & 0.17 & 10 & 178 & 246 & 431 \\
\hline
\end{tabular}

Note: $\mathrm{CaCO}_{3}=$ carbonate content $\mathrm{C}_{\text {tot }}=$ total carbon; $\mathrm{C}_{\mathrm{org}}=$ total organic carbon as weight percent of the carbonate-free sample; $\mathrm{C}_{\text {org }}=$ total organic carbon as weight percent of the whole sample; $\mathrm{MOM}=$ the amount of marine organic carbon in percent of the total organic carbon; $\mathrm{N}_{\text {tot }}=$ total nitrogen; $\mathrm{C} / \mathrm{N}=$ organic carbon/total nitrogen ratios; $\mathrm{HI}=$ hydrogen index; $\mathrm{OI}=$ oxygen index; and $\mathrm{T}_{\max }=$ temperature of maximum pyrolysis yield. (MOM = rough estimates based on the correlation between hydrogen indexes and the maceral composition shown for DSDP sediments in Stein et al., 1986.)

657

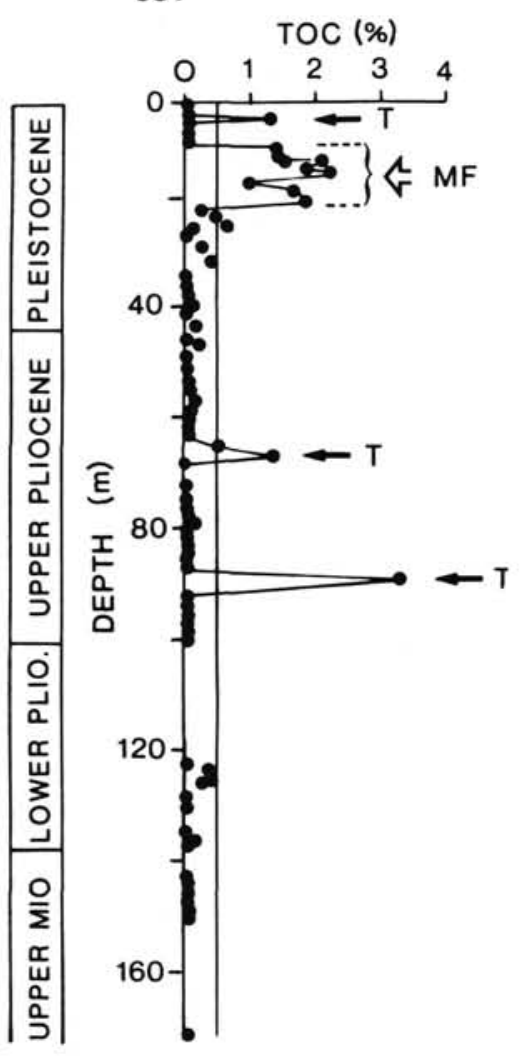

MF mudflow
$T$ turbidite
659

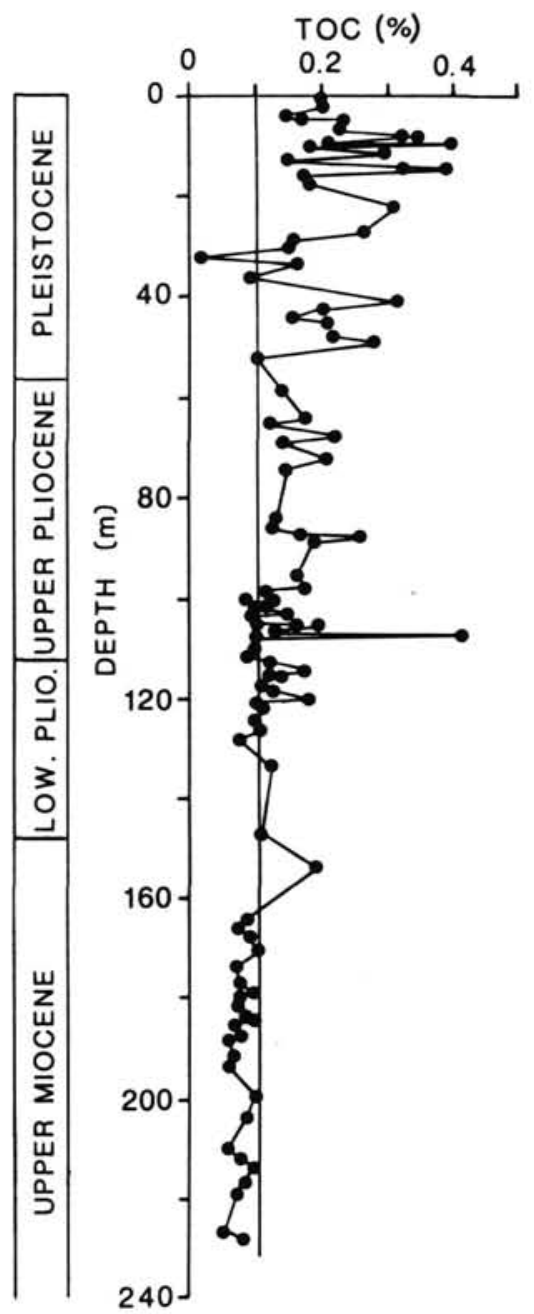

Figure 5. Total organic carbon contents at Sites 657 and 659. The record of Site 657 is based on shipboard data, and that of Site 659 on the data in Table 2. 
Table 2. Summary of data from Site 659.

\begin{tabular}{|c|c|c|c|c|c|c|c|}
\hline $\begin{array}{l}\text { Core, section, } \\
\text { interval }(\mathrm{cm})\end{array}$ & $\begin{array}{l}\text { Depth } \\
\text { (mbsf) }\end{array}$ & $\mathrm{C}_{\text {tot }}$ & $\mathrm{C}_{\text {org' }}$ & $\mathrm{C}_{\text {org }}$ & $\mathrm{CaCO}_{3}$ & $\mathrm{~N}_{\text {tot }}$ & $\mathrm{C} / \mathrm{N}$ \\
\hline \multicolumn{8}{|l|}{$108-659 \mathrm{~A}-$} \\
\hline $1 \mathrm{H}-1,40$ & 0.40 & 7.87 & 0.55 & 0.20 & 63.9 & 0.04 & 6 \\
\hline $1 \mathrm{H}-2,40$ & 1.90 & 7.47 & & & & & \\
\hline $1 \mathrm{H}-3,40$ & 3.40 & 9.28 & 0.69 & 0.17 & 76.0 & 0.03 & 6 \\
\hline $2 \mathrm{H}-1,40$ & 8.20 & 5.98 & 0.61 & 0.32 & 47.2 & 0.05 & 7 \\
\hline $2 \mathrm{H}-2,40$ & 9.70 & 7.69 & 1.01 & 0.40 & 60.8 & 0.05 & 8 \\
\hline $2 \mathrm{H}-4,40$ & 12.70 & 6.69 & & & & & \\
\hline $2 \mathrm{H}-5,40$ & 14.20 & 6.50 & 0.66 & 0.32 & 51.5 & 0.04 & 7 \\
\hline $3 \mathrm{H}-3,40$ & 20.70 & 9.79 & & & & & \\
\hline $3 \mathrm{H}-4,40$ & 22.20 & 5.31 & 0.53 & 0.31 & 41.7 & 0.04 & 8 \\
\hline $3 \mathrm{H}-5,40$ & 23.70 & 9.73 & & & & & \\
\hline $3 \mathrm{H}-6,40$ & 25.20 & 5.31 & & & & & \\
\hline $4 \mathrm{H}-1,40$ & 27.20 & 7.10 & 0.61 & 0.26 & 57.0 & 0.03 & 8 \\
\hline $4 \mathrm{H}-2,40$ & 28.70 & 10.24 & 0.97 & 0.15 & 84.1 & 0.02 & 8 \\
\hline $4 \mathrm{H}-4,40$ & 31.70 & 9.81 & & & & & \\
\hline $5 \mathrm{H}-1,40$ & 36.70 & 4.96 & & & & & \\
\hline $5 \mathrm{H}-2,40$ & 38.20 & 5.78 & & & & & \\
\hline $5 \mathrm{H}-4,40$ & 41.20 & 7.08 & 0.72 & 0.31 & 56.4 & 0.03 & 9 \\
\hline $5 \mathrm{H}-5,40$ & 42.70 & 9.99 & 1.10 & 0.20 & 81.6 & 0.03 & 8 \\
\hline $5 \mathrm{H}-6,40$ & 44.20 & 6.65 & 0.34 & 0.16 & 54.1 & 0.02 & 7 \\
\hline $7 \mathrm{H}-1,40$ & 55.70 & 8.15 & 0.46 & 0.15 & 66.6 & 0.03 & 5 \\
\hline $7 \mathrm{H}-3,40$ & 58.70 & 9.18 & 0.56 & 0.14 & 75.4 & 0.02 & 6 \\
\hline $7 \mathrm{H}-4,40$ & 60.15 & 9.03 & & & & & \\
\hline $7 \mathrm{H}-5,40$ & 61.65 & 9.22 & & & & & \\
\hline $7 \mathrm{H}-6,40$ & 63.15 & 7.35 & & & & & \\
\hline $7 \mathrm{H}-7,25$ & 64.50 & 8.16 & 0.52 & 0.17 & 66.6 & 0.02 & 9 \\
\hline $8 \mathrm{H}-1,63$ & 65.46 & 9.76 & 0.61 & 0.12 & 80.3 & 0.02 & 6 \\
\hline $8 \mathrm{H}-2,12$ & 66.42 & 7.73 & & & & & \\
\hline $8 \mathrm{H}-3,12$ & 67.92 & 10.26 & 1.35 & 0.22 & 83.7 & 0.02 & 10 \\
\hline $8 \mathrm{H}-4,12$ & 69.42 & 9.48 & 0.64 & 0.14 & 77.8 & 0.03 & 5 \\
\hline $8 \mathrm{H}-6,12$ & 72.42 & 10.35 & 1.34 & 0.21 & 84.5 & 0.03 & 7 \\
\hline $9 \mathrm{H}-1,40$ & 74.80 & 10.13 & 0.88 & 0.15 & 83.2 & 0.02 & 7 \\
\hline $9 \mathrm{H}-2,40$ & 76.20 & 10.35 & & & & & \\
\hline $9 \mathrm{H}-3,40$ & 77.70 & 7.81 & & & & & \\
\hline $9 \mathrm{H}-4,40$ & 79.20 & 9.51 & & & & & \\
\hline $9 \mathrm{H}-6,40$ & 82.20 & 10.30 & & & & & \\
\hline $10 \mathrm{H}-1,40$ & 84.20 & 10.26 & 0.84 & 0.13 & 84.4 & 0.02 & 6 \\
\hline $10 \mathrm{H}-2,40$ & 85.70 & 9.90 & 0.67 & 0.12 & 81.5 & 0.02 & 7 \\
\hline $10 \mathrm{H}-3,40$ & 87.20 & 10.32 & & & & & \\
\hline $10 \mathrm{H}-3,85$ & 87.65 & 9.00 & 0.64 & 0.17 & 73.6 & 0.02 & 7 \\
\hline $10 \mathrm{H}-3,120$ & 88.00 & 9.64 & 1.19 & 0.26 & 78.2 & 0.02 & 11 \\
\hline $10 \mathrm{H}-3,130$ & 88.10 & 8.15 & & & & & \\
\hline $10 \mathrm{H}-4,40$ & 88.70 & 9.54 & 0.86 & 0.19 & 77.9 & 0.02 & 11 \\
\hline $10 \mathrm{H}-5,40$ & 90.20 & 10.41 & & & & & \\
\hline $11 \mathrm{H}-2,40$ & 95.20 & 8.71 & 0.56 & 0.16 & 71.2 & 0.02 & 7 \\
\hline $11 \mathrm{H}-4,40$ & 98.20 & 8.71 & 0.61 & 0.18 & 71.1 & 0.03 & 6 \\
\hline $11 \mathrm{H}-5,40$ & 99.70 & 9.64 & 0.60 & 0.12 & 79.3 & 0.01 & 9 \\
\hline $11 \mathrm{H}-6,40$ & 101.20 & 10.00 & & & & & \\
\hline $12 \mathrm{H}-1,40$ & 103.20 & 10.01 & 0.55 & 0.10 & 82.6 & 0.01 & 8 \\
\hline $13 \mathrm{H}-3,40$ & 115.70 & 9.27 & 0.52 & 0.12 & 76.2 & 0.03 & 5 \\
\hline $13 \mathrm{H}-5,40$ & 118.70 & 10.52 & 0.92 & 0.12 & 86.7 & 0.01 & 9 \\
\hline $13 \mathrm{H}-6,37$ & 120.17 & 7.30 & 0.45 & 0.18 & 59.3 & 0.02 & 8 \\
\hline $13 \mathrm{H}-7,17$ & 121.47 & 7.90 & 0.29 & 0.10 & 65.0 & 0.03 & 3 \\
\hline $14 \mathrm{H}-1,40$ & 122.20 & 10.40 & 0.77 & 0.11 & 85.8 & 0.02 & 6 \\
\hline $14 \mathrm{H}-2,40$ & 123.70 & 8.62 & & & & & \\
\hline $14 \mathrm{H}-3,32$ & 125.12 & 9.30 & 0.44 & 0.10 & 76.6 & 0.02 & 6 \\
\hline $14 \mathrm{H}-4,40$ & 126.70 & 10.70 & 0.94 & 0.11 & 88.3 & 0.02 & 6 \\
\hline $14 \mathrm{H}-5,40$ & 128.20 & 9.82 & 0.41 & 0.08 & 81.2 & 0.01 & 14 \\
\hline $14 \mathrm{H}-6,40$ & 129.70 & 9.72 & & & & & \\
\hline $15 \mathrm{H}-2,40$ & 133.20 & 10.56 & 0.96 & 0.13 & 87.0 & & \\
\hline $15 \mathrm{H}-4,40$ & 136.20 & 10.57 & & & & & \\
\hline $16 \mathrm{H}-2,40$ & 142.70 & 9.61 & & & & & \\
\hline $16 \mathrm{H}-5,40$ & 147.20 & 9.00 & 0.42 & 0.11 & 74.1 & 0.02 & 5 \\
\hline $17 \mathrm{H}-3,45$ & 153.75 & 10.13 & 1.12 & 0.19 & 82.8 & 0.02 & 12 \\
\hline $17 \mathrm{H}-4,41$ & 155.21 & 10.73 & 1.05 & 0.12 & 88.4 & 0.02 & 6 \\
\hline $19 \mathrm{H}-2,40$ & 171.13 & & 0.57 & & & & 5 \\
\hline $20 \mathrm{H}-3,43$ & 182.16 & 7.39 & 0.19 & 0.07 & 61.0 & 0.02 & 4 \\
\hline $20 \mathrm{H}-5,44$ & 185.11 & 5.52 & 0.13 & 0.07 & 45.4 & 0.03 & 3 \\
\hline $20 \mathrm{H}-6,41$ & 186.54 & 7.42 & 0.18 & 0.07 & 61.3 & 0.03 & 3 \\
\hline $20 \mathrm{H}-7,39$ & 188.02 & 5.97 & 0.14 & 0.07 & 49.2 & 0.03 & 2 \\
\hline $22 X-2,40$ & 199.70 & 5.41 & 0.18 & 0.10 & 44.3 & 0.04 & 3 \\
\hline $22 \times-3,40$ & 201.20 & 0.52 & 0.13 & 0.13 & 3.3 & 0.07 & 2 \\
\hline $22 X-5,20$ & 204.00 & 4.47 & 0.14 & 0.09 & 36.5 & 0.04 & 2 \\
\hline $23 \mathrm{X}-3,20$ & 210.50 & 6.82 & 0.14 & 0.06 & 56.3 & 0.02 & 3 \\
\hline $23 X-4,20$ & 212.00 & 1.21 & 0.09 & 0.08 & 9.4 & 0.04 & 2 \\
\hline $23 X-5,20$ & 213.50 & 5.12 & 0.17 & 0.10 & 41.8 & 0.05 & 2 \\
\hline $23 \mathrm{X}-6,20$ & 215.00 & 5.79 & 0.19 & 0.10 & 47.4 & 0.02 & 5 \\
\hline $24 X-1,45$ & 217.25 & 5.18 & 0.15 & 0.09 & 42.5 & 0.04 & 2 \\
\hline
\end{tabular}

Table 2 (continued).

\begin{tabular}{cccccccr}
\hline $\begin{array}{c}\text { Core, section, } \\
\text { interval (cm) }\end{array}$ & $\begin{array}{c}\text { Depth } \\
\text { (mbsf) }\end{array}$ & $\mathrm{C}_{\text {tot }}$ & $\mathrm{C}_{\text {org' }}$ & $\mathrm{C}_{\text {org }}$ & $\mathrm{CaCO}_{3}$ & $\mathrm{~N}_{\text {tot }}$ & $\mathrm{C} / \mathrm{N}$ \\
\hline $24 \mathrm{X}-2,22$ & 218.52 & 5.71 & 0.14 & 0.07 & 47.0 & 0.03 & 2 \\
$25 \mathrm{X}-1,40$ & 226.70 & 4.64 & 0.09 & 0.06 & 38.2 & 0.02 & 3 \\
$25 \mathrm{X}-2,40$ & 228.20 & 5.55 & 0.16 & 0.09 & 45.5 & 0.04 & 2
\end{tabular}

108-659B-

$\begin{array}{lrrlllll}4 \mathrm{H}-4,4 & 30.14 & 7.08 & 0.36 & 0.15 & 57.7 & 0.02 & 7 \\ 4 \mathrm{H}-5,40 & 32.00 & 10.19 & 0.10 & 0.02 & 84.8 & & \\ 4 \mathrm{H}-6,40 & 33.50 & 6.44 & 0.34 & 0.16 & 52.3 & 0.03 & 6 \\ 5 \mathrm{H}-5,40 & 35.00 & 8.34 & & & & & \\ 5 \mathrm{H}-6,40 & 36.50 & 9.33 & 0.40 & 0.09 & 77.0 & 0.03 & 4 \\ 6 \mathrm{H}-1,40 & 45.00 & 8.96 & 0.77 & 0.21 & 72.9 & 0.02 & 9 \\ 6 \mathrm{H}-3,40 & 48.00 & 7.55 & 0.55 & 0.21 & 61.1 & 0.03 & 8 \\ 6 \mathrm{H}-4,40 & 49.50 & 6.11 & 0.54 & 0.28 & 48.6 & 0.03 & 9 \\ 6 \mathrm{H}-5,40 & 51.00 & 10.21 & & & & & \\ 6 \mathrm{H}-6,40 & 52.50 & 9.70 & 0.50 & 0.10 & 80.0 & 0.01 & 7 \\ 12 \mathrm{H}-1,66 & 98.26 & 8.71 & 0.40 & 0.11 & 71.6 & 0.02 & 7 \\ 12 \mathrm{H}-2,66 & 99.76 & 9.55 & 0.51 & 0.11 & 78.7 & 0.02 & 6 \\ 12 \mathrm{H}-3,66 & 101.26 & 8.40 & 0.39 & 0.12 & 69.0 & 0.02 & 6 \\ 12 \mathrm{H}-4,66 & 102.76 & 6.19 & 0.19 & 0.09 & 50.8 & 0.01 & 6 \\ 12 \mathrm{H}-5,66 & 104.26 & 9.43 & & & & & \\ 12 \mathrm{H}-6,66 & 105.76 & 6.95 & 0.37 & 0.16 & 56.6 & 0.02 & 7 \\ 13 \mathrm{H}-1,40 & 107.50 & 5.57 & 0.72 & 0.41 & 43.0 & 0.03 & 12 \\ 13 \mathrm{H}-2,40 & 109.00 & 10.35 & & & & & \\ 13 \mathrm{H}-3,41 & 110.51 & 6.29 & & & & & \\ 19 \mathrm{H}-1,40 & 164.50 & 6.27 & 0.18 & 0.09 & 51.5 & 0.01 & 6 \\ 19 \mathrm{H}-2,38 & 165.98 & 7.89 & 0.22 & 0.08 & 65.1 & 0.02 & 4 \\ 19 \mathrm{H}-3,38 & 167.48 & 9.10 & 0.36 & 0.09 & 75.1 & 0.02 & 5 \\ 19 \mathrm{H}-4,38 & 168.98 & 9.94 & & & & & \\ 19 \mathrm{H}-5,38 & 170.48 & 7.94 & 0.30 & 0.10 & 65.3 & 0.03 & 3 \\ 19 \mathrm{H}-6,38 & 171.98 & 7.08 & & & & & \\ 20 \mathrm{H}-1,37 & 173.97 & 8.66 & 0.26 & 0.07 & 71.6 & 0.02 & 4 \\ 20 \mathrm{H}-2,37 & 175.47 & 6.64 & & & & & \\ 20 \mathrm{H}-3,37 & 176.97 & 7.37 & 0.19 & 0.07 & 60.8 & & \\ 20 \mathrm{H}-4,37 & 178.47 & 3.95 & 0.12 & 0.08 & 32.2 & 0.02 & 4 \\ & & & & & & & \end{array}$

$108-659 \mathrm{C}$ -

\begin{tabular}{|c|c|c|c|c|c|c|c|}
\hline $1 \mathrm{H}-2,62$ & 2.12 & 7.51 & 0.52 & 0.20 & 60.9 & 0.02 & 10 \\
\hline $1 \mathrm{H}-3,62$ & 3.62 & 5.94 & 0.28 & 0.14 & 48.3 & 0.03 & 6 \\
\hline $1 \mathrm{H}-4,62$ & 5.12 & 5.56 & 0.41 & 0.23 & 44.4 & 0.02 & 10 \\
\hline $1 \mathrm{H}-5,62$ & 6.62 & 6.57 & 0.48 & 0.23 & 52.9 & 0.02 & 12 \\
\hline $1 \mathrm{H}-6,62$ & 8.12 & 7.57 & 0.87 & 0.35 & 60.2 & 0.02 & 15 \\
\hline $1 \mathrm{H}-7,23$ & 9.23 & 8.80 & 0.73 & 0.21 & 71.6 & 0.02 & 9 \\
\hline $2 \mathrm{H}-1,40$ & 9.90 & 6.39 & 0.37 & 0.18 & 51.8 & 0.02 & 9 \\
\hline $2 \mathrm{H}-2,40$ & 11.40 & 7.24 & 0.70 & 0.29 & 57.9 & 0.02 & 14 \\
\hline $2 \mathrm{H}-3,40$ & 12.90 & 9.40 & 0.64 & 0.15 & 77.1 & 0.02 & 9 \\
\hline $2 \mathrm{H}-4,40$ & 14.40 & 6.94 & 0.86 & 0.39 & 54.6 & 0.03 & 14 \\
\hline $2 \mathrm{H}-5,40$ & 15.90 & 8.22 & 0.51 & 0.17 & 67.1 & 0.02 & 9 \\
\hline $2 \mathrm{H}-6,40$ & 17.40 & 9.56 & 0.81 & 0.18 & 78.2 & 0.02 & 10 \\
\hline $5 \mathrm{H}-1,40$ & 100.40 & 10.38 & 0.62 & 0.09 & 85.8 & 0.01 & 9 \\
\hline $5 \mathrm{H}-2,40$ & 101.90 & 8.80 & 0.35 & 0.10 & 72.5 & 0.01 & 9 \\
\hline $5 \mathrm{H}-3,40$ & 103.40 & 7.00 & 0.35 & 0.15 & 57.1 & 0.02 & 9 \\
\hline $5 \mathrm{H}-4,40$ & 104.90 & 9.28 & 0.42 & 0.10 & 76.5 & 0.01 & 8 \\
\hline $5 \mathrm{H}-4,94$ & 105.44 & 4.24 & 0.29 & 0.19 & 33.7 & 0.02 & 10 \\
\hline $5 \mathrm{H}-5,40$ & 106.40 & 4.42 & 0.21 & 0.14 & 35.7 & 0.02 & 7 \\
\hline $5 \mathrm{H}-6,40$ & 107.90 & 9.43 & 0.45 & 0.10 & 77.8 & 0.01 & 8 \\
\hline $6 \mathrm{H}-1,40$ & 109.90 & 8.32 & 0.32 & 0.10 & 68.5 & 0.01 & 8 \\
\hline $6 \mathrm{H}-2,40$ & 111.40 & 8.58 & 0.30 & 0.09 & 70.8 & 0.01 & 8 \\
\hline $6 \mathrm{H}-3,40$ & 112.90 & 9.83 & 0.64 & 0.12 & 80.9 & 0.01 & 11 \\
\hline $6 \mathrm{H}-4,40$ & 114.40 & 6.13 & 0.34 & 0.17 & 49.7 & 0.02 & 11 \\
\hline $6 \mathrm{H}-5,40$ & 115.90 & 10.35 & 0.90 & 0.13 & 85.1 & 0.01 & 10 \\
\hline $6 \mathrm{H}-6,40$ & 117.40 & 6.52 & 0.24 & 0.11 & 53.4 & 0.01 & 8 \\
\hline $7 \mathrm{H}-2,44$ & 178.94 & 5.57 & 0.18 & 0.10 & 45.6 & 0.02 & 5 \\
\hline $7 \mathrm{H}-3,40$ & 180.40 & 7.09 & 0.19 & 0.08 & 58.4 & 0.03 & 2 \\
\hline $7 \mathrm{H}-4,40$ & 181.90 & 5.90 & 0.15 & 0.08 & 48.5 & 0.02 & 5 \\
\hline $7 \mathrm{H}-5,40$ & 183.40 & 7.57 & 0.23 & 0.09 & 62.4 & 0.02 & 6 \\
\hline $7 \mathrm{H}-6,40$ & 184.90 & 6.87 & 0.23 & 0.10 & 56.4 & 0.02 & 6 \\
\hline $8 \mathrm{H}-1,40$ & 186.90 & 2.09 & 0.09 & 0.07 & 16.8 & 0.02 & 5 \\
\hline $8 \mathrm{H}-2,40$ & 188.40 & 7.80 & 0.17 & 0.06 & 64.5 & 0.01 & 6 \\
\hline $8 \mathrm{H}-3,40$ & 189.90 & 5.96 & 0.75 & 0.40 & 46.3 & 0.02 & 25 \\
\hline $8 \mathrm{H}-4,40$ & 191.40 & 7.83 & 0.19 & 0.07 & 64.7 & 0.01 & 5 \\
\hline $8 \mathrm{H}-5,40$ & 192.90 & 7.95 & 0.20 & 0.07 & 65.7 & 0.01 & 5 \\
\hline $8 \mathrm{H}-6,40$ & 194.40 & 7.22 & 0.15 & 0.06 & 59.7 & 0.02 & 4 \\
\hline
\end{tabular}


Table 3. Results from Rock-Eval pyrolysis and CHN analyses from

658

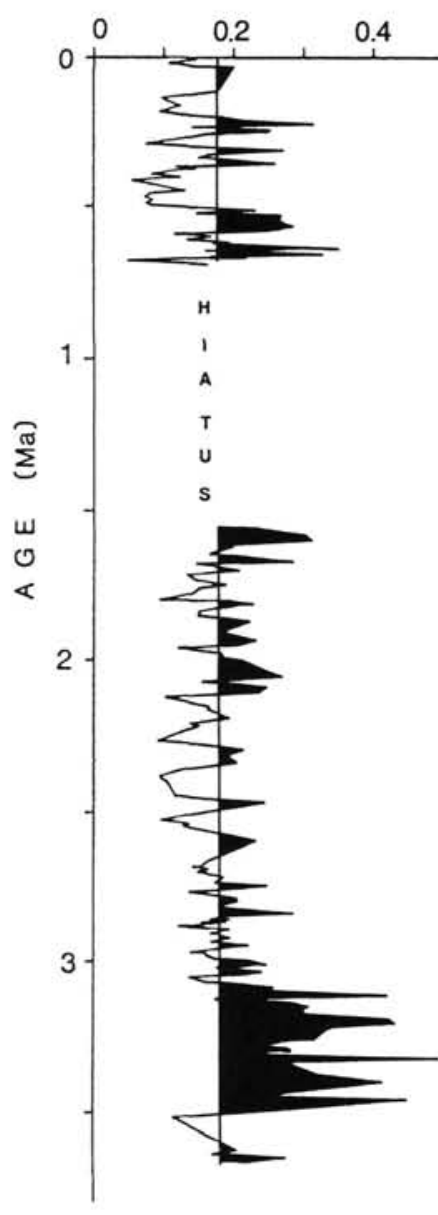

659

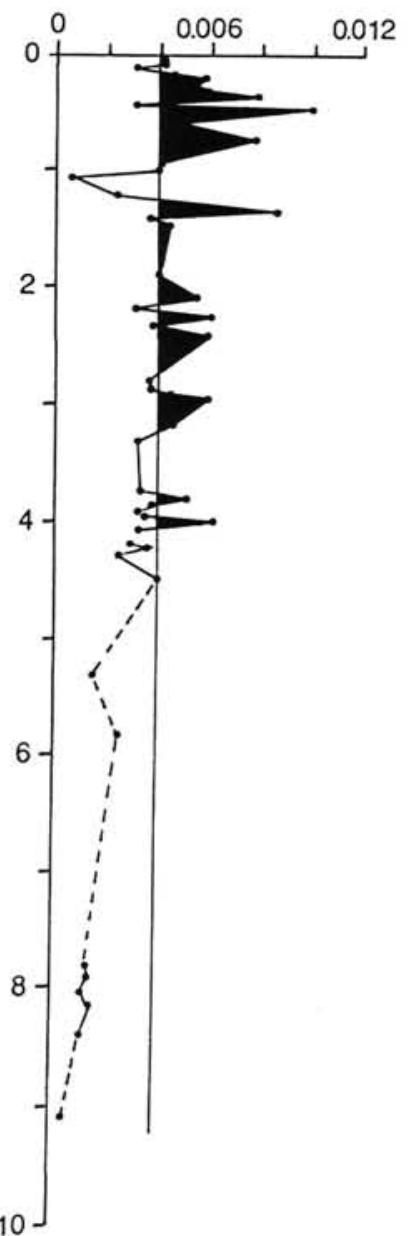

Figure 6. Accumulation rates of total organic carbon at Sites 658 and 659. The record of Site 658 is based on the detailed sedimentation rate data from Sarnthein and Tiedemann (this vol.).

\section{Organic Carbon/Nitrogen Ratios}

Different groups of organisms produce organic matter with different carbon and nitrogen contents. The mean organic carbon/nitrogen $(\mathrm{C} / \mathrm{N})$ ratio of marine zooplanktons and phytoplanktons is about 6 , whereas higher land plants are characterized by C/N ratios of more than 15 (Bordowskiy, 1965a, 1965b; Scheffer and Schachtschabel, 1984). Thus, C/N ratios of deep-sea sediments may give some information about the composition of organic matter preserved in these sediments.

At Site $658, \mathrm{C} / \mathrm{N}$ ratios vary between 5 and 30 , with most values between 6 and 15 (Figs. 8 and 9 and Table 1). Slightly lower values were found in sediments representing the uppermost 0.7 Ma (Fig. 8), suggesting some higher amounts of marine organic carbon in these sediments. Thus, the $\mathrm{C} / \mathrm{N}$ ratios also indicate that a mixed marine/terrigenous type of organic matter $(\mathrm{C} / \mathrm{N}$ ratios around 10$)$ is dominant in Site 658 sediments. However, samples with a higher marine portion $(\mathrm{C} / \mathrm{N}$ ratios around 5) and samples with a higher terrigenous portion $(\mathrm{C} / \mathrm{N}$ ratios $>>15)$ are common.

At Site 659 , most of the $\mathrm{C} / \mathrm{N}$ ratios vary between 2 and 10 (Fig. 9), which may suggest high amounts of marine organic

sediments (mainly turbiditic sequences and slump interval) at Site 657.

\begin{tabular}{|c|c|c|c|c|c|c|c|}
\hline $\begin{array}{l}\text { Core, section, } \\
\text { interval }(\mathrm{cm})\end{array}$ & $\begin{array}{l}\text { Depth } \\
\text { (mbsf) }\end{array}$ & $\mathrm{C}_{\text {org }}$ & HI & $T_{\max }$ & $\mathrm{N}_{\text {tot }}$ & $\mathrm{C} / \mathrm{N}$ & Remarks \\
\hline \multicolumn{8}{|l|}{ 108-657A- } \\
\hline $1 \mathrm{H}-4,109$ & 5.59 & 1.32 & & & 0.12 & 11 & Turbidite \\
\hline $2 \mathrm{H}-3,25$ & 10.37 & 0.73 & & & 0.05 & 15 & \\
\hline $2 \mathrm{H}-3,121$ & 11.33 & 1.41 & 164 & 413 & & & \\
\hline $2 \mathrm{H}-4,17$ & 11.79 & 1.75 & & & 0.13 & 13 & \\
\hline $2 \mathrm{H}-4,25$ & 11.87 & 1.93 & & & 0.16 & 12 & \\
\hline $2 \mathrm{H}-4,121$ & 12.80 & 1.47 & 315 & 408 & & & \\
\hline $2 \mathrm{H}-5,115$ & 14.21 & 1.74 & 297 & 411 & & & \\
\hline $2 \mathrm{H}-6,40$ & 14.95 & 2.24 & 324 & 412 & & & Slump \\
\hline $2 \mathrm{H}-7,23$ & 17.03 & 1.95 & & & 0.18 & 11 & \\
\hline $3 \mathrm{H}-1,121$ & 17.89 & 0.98 & 281 & 411 & & & \\
\hline $3 \mathrm{H}-2,25$ & 18.43 & 2.49 & & & 0.19 & 13 & \\
\hline $3 \mathrm{H}-3,121$ & 20.83 & 1.87 & 261 & 413 & & & \\
\hline $3 \mathrm{H}-5,121$ & 23.77 & 0.51 & 216 & 411 & & & \\
\hline $3 \mathrm{H}-6,25$ & 24.31 & 0.65 & & & 0.06 & 11 & \\
\hline $4 \mathrm{H}-4,120$ & 31.84 & 0.45 & 124 & 382 & & & Pelagic \\
\hline $8 \mathrm{H}-1,120$ & 65.40 & 0.54 & 96 & 390 & & & Pelagic \\
\hline $10 \mathrm{H}-4,143$ & 89.13 & 3.31 & & & 0.25 & 13 & Turbidite \\
\hline
\end{tabular}

Note: $\mathrm{C}_{\text {org }}=$ total organic carbon as weight percent of the whole sample; $\mathrm{HI}$ $=$ hydrogen index values (in $\mathrm{mgHC} / \mathrm{gC}$ ); $\mathrm{T}_{\max }=$ temperatures of maximum pyrolysis yield $\left({ }^{\circ} \mathrm{C}\right) ; \mathrm{N}_{\text {tot }}=$ total nitrogen; and $\mathrm{C} / \mathrm{N}=$ organic carbon/total nitrogen ratios.

Table 4. Results from Rock-Eval pyrolysis of sediment samples from Site 659.

\begin{tabular}{lclrr}
\hline $\begin{array}{l}\text { Core, section, } \\
\text { interval (cm) }\end{array}$ & $\begin{array}{l}\text { Depth } \\
\text { (mbsf) }\end{array}$ & $\begin{array}{l}\text { Total } \\
\text { organic } \\
\text { carbon }\end{array}$ & HI & $\mathrm{T}_{\text {max }}$ \\
\hline $120-659 \mathrm{~A}-$ & & & & \\
& & & 52 & 404 \\
$5 \mathrm{H}-3,23$ & 39.53 & 0.23 & 63 & 399 \\
$5 \mathrm{H}-3,27$ & 39.57 & 0.38 & 66 & 395 \\
$5 \mathrm{H}-3,32$ & 39.62 & 0.4 & 71 & - \\
$14 \mathrm{H}-3,40$ & 125.2 & 0.14 & 121 & 422 \\
$14 \mathrm{H}-3,47$ & 125.27 & 1.2 & 83 & - \\
$23 \mathrm{H}-5,85$ & 214.35 & 0.06 & 120 & - \\
$23 \mathrm{H}-5,99$ & 214.49 & 0.11 & \\
\hline
\end{tabular}

Note: $\mathrm{HI}=$ hydrogen index values $(\mathrm{mgHC} / \mathrm{gC})$ and $\mathrm{T}_{\max }=$ temperatures of maximum pyrolysis yield $\left({ }^{\circ} \mathrm{C}\right)$.

material (see above). However, the $\mathrm{C} / \mathrm{N}$ data of these organic-carbon-lean sediments of Site 659 have to be interpreted with extreme caution. For example, in organiccarbon-lean sediments, the amount of inorganic nitrogen (fixed as ammonium ions in the interlayers of clay minerals) may become a major portion of the total nitrogen (e.g., Stevenson and Cheng, 1972; Müller, 1977), causing (too) low $\mathrm{C} / \mathrm{N}$ ratios. This can explain the very low $\mathrm{C} / \mathrm{N}$ ratios of about 2 in the late Miocene interval of Site 659 , which coincide with extremely low TOC values around $0.07 \%$ (Table 2 and Fig. 5). Hence, these $\mathrm{C} / \mathrm{N}$ data should not be interpreted as an indicator of the quality of the organic matter.

\section{Kerogen Microscopy}

Kerogen microscopy analyses were performed on a selected set of 18 samples from Site 658 , covering the entire depth range at that site (Fig. 10 and Table 5). No detailed maceral analyses were performed on material from Sites 657 and 659 because of the low TOC contents, but kerogen microscopy of two samples from Site 659 indicates that 


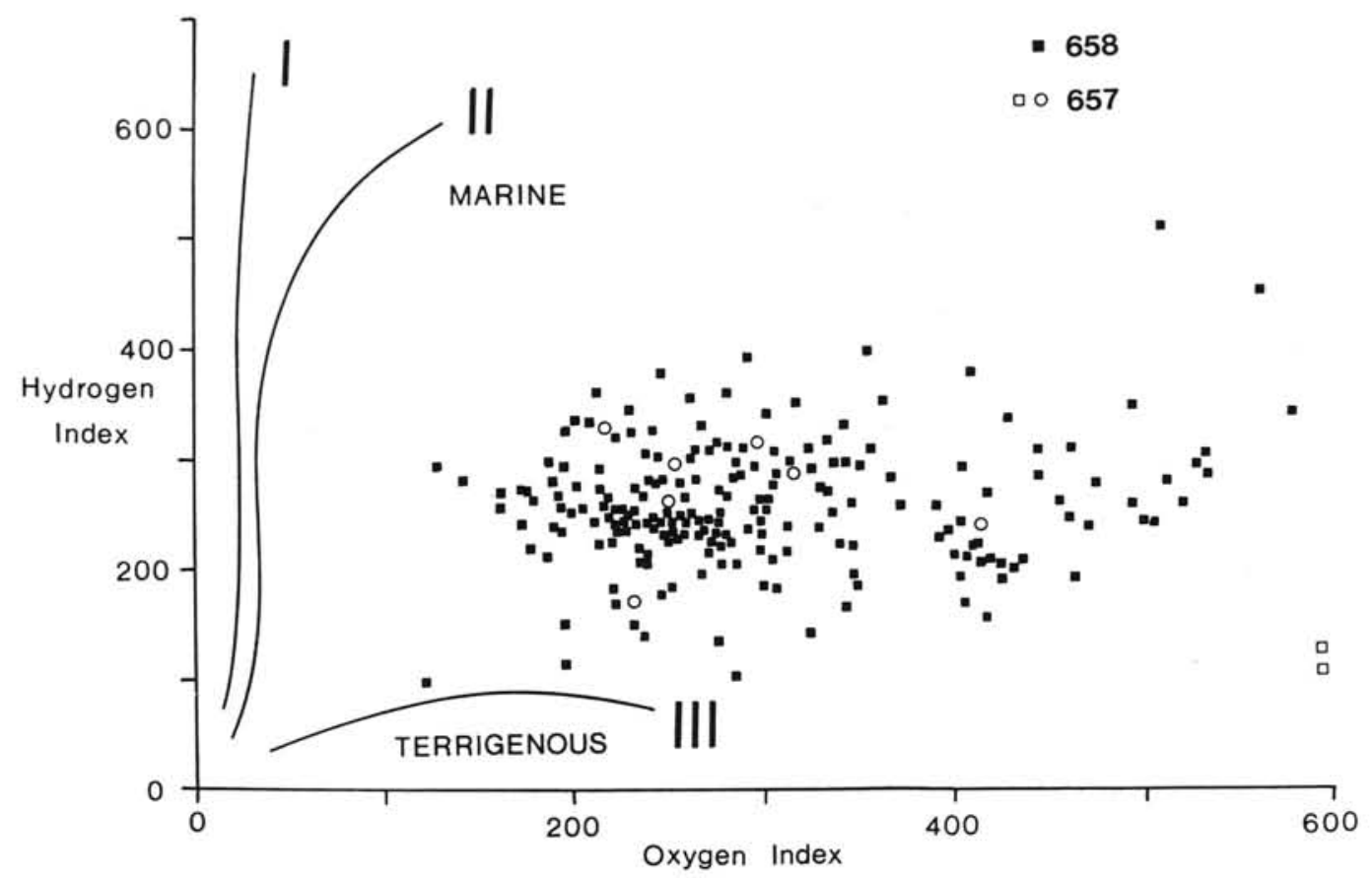

Figure 7. Results of Rock-Eval pyrolysis displayed as hydrogen index vs. oxygen index diagram (Espitalié et al., 1977). Black squares are data from Site 658. Open squares are from pelagic sediments of Site 657, open circles are from the slump interval of Site 657.

terrigenous organic matter predominates over marine organic matter.

The main macerals in the organic matter fractions of these samples from Site 658 are alginite, liptodetrinite, inertinite, and vitrinite (Fig. 10 and Table 5). Sporinite, common in near-shore sediments from other locations in the Atlantic (Cornford et al., 1979; Rullkötter et al., 1980, 1984; Stein et al., 1989), was not observed in Site 658 sediments, with one exception: in Sample 108-658A-14H-7, $34 \mathrm{~cm}$, minor amounts of $2 \%$ sporinite occur (Table 5 ).

The amounts of the main marine maceral alginite vary between $12 \%$ and $60 \%$ of the total macerals. Liptodetrinites (liptinites too small to be classified into a specific liptinite group) range between $11 \%$ and $46 \%$. Because alginite is the only liptinite identified in major amounts (i.e., terrigenous liptinites such as sporinites are virtually absent), we suggest that the liptodetrinites mainly represent marine organic material. Thus, the total amount of marine organic matter (alginite plus liptodetrinite) ranges between $30 \%$ and $80 \%$ (Fig. 10 and Table 5), which corroborates Rock-Eval and $\mathrm{C} / \mathrm{N}$ results (Table 1). Atomic $\mathrm{H} / \mathrm{C}$ ratios of 1.0-1.5 suggest the dominance of marine organic matter (Table 6; cf. Tissot and Welte, 1984).

The terrigenous macerals are dominated by inertinites; vitrinites are of secondary importance (Fig. 10). Inertinites occur in abundances between $15 \%$ and $45 \%$, and vitrinites vary between $0 \%$ and $18 \%$ (Table 5). Only the two lowermost samples (120-658A-30X-1, $44 \mathrm{~cm}$, and 120-658A-33X-2, 109 $\mathrm{cm})$, which also have a larger amount of total terrigenous components $(60 \%)$, show a somewhat higher content of vitrinite ( $40 \%$ and $27 \%$, respectively; Table 5$)$. The dominance of inertinites may point toward a stronger degradation (oxidation) of the terrigenous plant fragments during transport (cf. Stein et al., 1989). However, input of reworked kerogen particles of older more mature strata can also explain the higher inertinite values (see below).

\section{Extractable Lipids}

A gas chromatogram (GC) of the "total lipid" fraction of Sample 658A-21-6, 7-14 cm, is shown in the upper half of Figure 11, while a GC of the aliphatic hydrocarbon fraction of the same sample is shown in the lower half. Long straightchain alkanes of terrigenous origin $\left(C_{2}-C_{33}\right)$ are the most abundant compounds in the aliphatic fraction, but they have minor importance in the total lipid fraction. Marine-derived long-chain unsaturated ketones and steroids are always the most important classes of compounds in the total lipid fraction of samples from Site 658 (for details of compound identification, see ten Haven et al., this vol.), but they have minor importance in the total lipid extracts from Sites 657 and 659 samples (not shown here). This testifies to the marine character of the organic matter at Site 658 .

Although estimates of the different sources of input (e.g. marine vs. terrigenous) can only be made on a semiquantitative level with the aid of biomarkers, it is clear from the GCs shown in Figure 11 that inspection of the aliphatic fraction alone would lead to an overestimate of terrigenous input. This is especially true for samples in which the early diagenetic conversion of steroids (from sterols via sterenes to steranes) is still not in an advanced stage. In the sample investigated here, sterols are still very abundant while sterenes (minor peaks eluting between $n-C_{28}$ and $n-C_{31}$; Fig. 11) are only present in subordinate amounts in the aliphatic hydrocarbon fraction.

\section{Maturity of the Organic Matter}

The low temperatures of maximum pyrolysis yield $\left(\mathrm{T}_{\max }\right)$, which vary from $400^{\circ} \mathrm{C}$ to $430^{\circ} \mathrm{C}$ (Fig. 12, and Tables 1,3 , and 4), indicate the low maturity of the organic matter at Sites 657 , 658 , and 659 . The few vitrinite reflectance values determined in samples from Site $658(92,221,225$, and $293 \mathrm{mbsf})$ are very low $(0.23 \%-0.27 \%$; Fig. 12 and Table 5) and support the immaturity. The low abundance of sterenes/steranes in the 


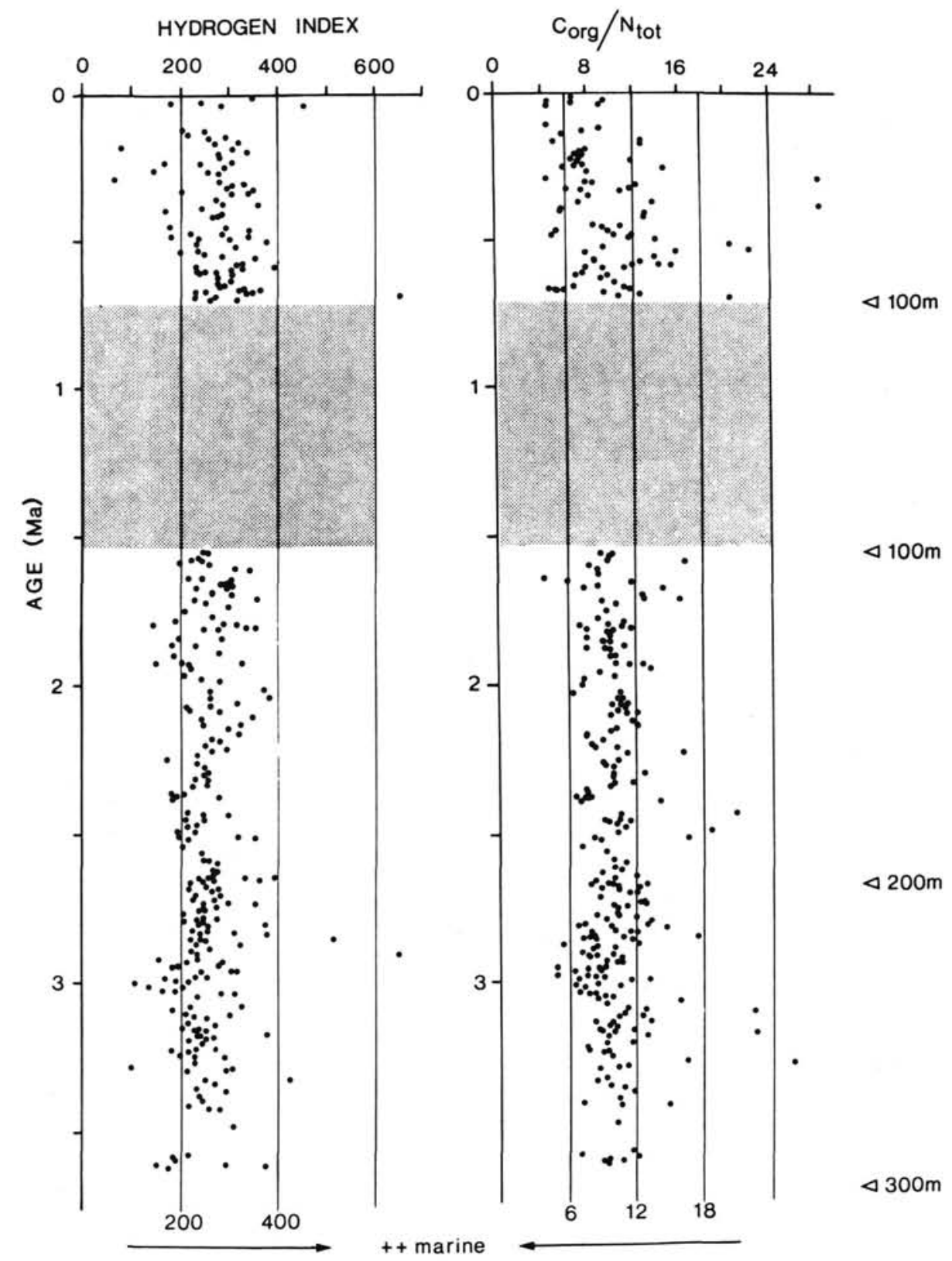

Figure 8. Hydrogen index $(\mathrm{HI})$ values and $\mathrm{C} / \mathrm{N}$ ratios from sediments of Site 658 . Increasing $\mathrm{HI}$ values and decreasing $\mathrm{C} / \mathrm{N}$ ratios imply an increasing amount of marine proportion in the organic matter, indicated by the black arrows.

hydrocarbon fraction and the high abundance of free extractable sterols also suggest that major diagenetic alterations of the organic matter have not yet occurred (see ten Haven et al., this vol.).

A second maximum in vitrinite reflectance (values around $0.5 \%$; Fig. 12) may imply the presence of redeposited material.

\section{DISCUSSION}

Our detailed study of organofacies types and accumulation rates of marine and terrigenous organic matter give important information about the evolution of both the paleoclimate in northwest Africa and the paleoceanography in the northeast Atlantic during late Neogene and Quaternary times. In addi- tion, evidence for sediment redeposition processes is available from the organic carbon data.

\section{Terrigenous Organic Carbon: Implications for Paleoclimate in Northwest Africa}

In general, the accumulation of organic carbon is mainly a function of rate of supply and/or rate of preservation. Since that part of the terrigenous organic matter that has survived long-distance transport to the sea is resistant to oxic decomposition (Tissot et al., 1979; Waples, 1983), changes in accumulation rates of terrigenous organic matter mainly reflect changes in the rates of supply rather than changes in preservation. Thus, these accumulation rates can be interpreted as an indicator of paleoclimate on the surrounding continent. 
Table 5. Composition of macerals and vitrinite reflectance values from sediments of Site 658.

\begin{tabular}{|c|c|c|c|c|c|c|c|c|c|c|}
\hline $\begin{array}{l}\text { Core, section, } \\
\text { interval }(\mathrm{cm})\end{array}$ & $\begin{array}{l}\text { Depth } \\
\text { (mbsf) }\end{array}$ & $\begin{array}{l}\text { Total } \\
\text { organic } \\
\text { carbon }\end{array}$ & Vitrinite & Inertinite & Sporinite & Liptodetrinite & Alginite & $\begin{array}{l}\text { Marine } \\
\text { organic } \\
\text { matter }\end{array}$ & Ro & Macerals/pyrite \\
\hline \multicolumn{11}{|l|}{ 108-658A- } \\
\hline $3 \mathrm{H}-1,120$ & 16.4 & 1.23 & 0 & 45 & 0 & 41 & 15 & 55 & & 0.20 \\
\hline $6 \mathrm{H}-1,84$ & 44.5 & 1.61 & 4 & 39 & 0 & 46 & 12 & 58 & & 0.25 \\
\hline $7 \mathrm{H}-2,120$ & 55.9 & 0.53 & 14 & 40 & 0 & 17 & 29 & 46 & & 0.03 \\
\hline $11 \mathrm{H}-1,120$ & 91.7 & & 4 & 15 & 0 & 37 & 44 & 81 & 0.25 & 0.62 \\
\hline $11 \mathrm{H}-3,102$ & 94.7 & 2.87 & 3 & 42 & 0 & 14 & 42 & 55 & & 0.18 \\
\hline $12 \mathrm{H}-2,118$ & 103.0 & 2.18 & 0 & 36 & 0 & 40 & 24 & 64 & & 0.05 \\
\hline $14 \mathrm{H}-7,34$ & 127.3 & 2.34 & 3 & 20 & 2 & 35 & 40 & 76 & & 0.51 \\
\hline $16 \mathrm{H}-4,120$ & 144.1 & 2.44 & 5 & 15 & 0 & 44 & 35 & 80 & & 0.40 \\
\hline $22 X-2,71$ & 188.3 & 2.81 & 18 & 28 & 0 & 27 & 27 & 54 & & 0.24 \\
\hline $24 \mathrm{X}-1,14$ & 205.5 & 1.65 & 7 & 42 & 0 & 17 & 35 & 52 & & 0.23 \\
\hline $25 X-5,65$ & 221.3 & 1.75 & 12 & 25 & 0 & 17 & 47 & 63 & 0.23 & 0.30 \\
\hline $26 \mathrm{X}-2,30$ & 225.7 & 1.31 & 15 & 41 & 0 & 26 & 18 & 44 & 0.27 & 0.53 \\
\hline $26 \mathrm{X}-4,86$ & 228.5 & 1.75 & 4 & 21 & 0 & 16 & 60 & 75 & & 0.58 \\
\hline $27 X-4,41$ & 238.7 & 2.66 & 16 & 53 & 0 & 11 & 21 & 32 & & 0.79 \\
\hline $27 X-5,18$ & 240.0 & 3.03 & 12 & 59 & 0 & 11 & 18 & 29 & & 0.94 \\
\hline $29 \mathrm{X}-8,38$ & 262.3 & 2.68 & 22 & 33 & 0 & 12 & 33 & 45 & & 0.35 \\
\hline $30 \mathrm{X}-1,44$ & 262.8 & 2.45 & 40 & 20 & 0 & 20 & 20 & 40 & & 0.38 \\
\hline $33 \mathrm{X}-2,109$ & 293.5 & 1.21 & 27 & 33 & 0 & 25 & 14 & 40 & 0.25 & 0.17 \\
\hline
\end{tabular}

Note: The amounts of vitrinite, inertinite, sporinite, liptodetrinite, and alginite are shown in percentages of the total macerals. The percentages of marine organic matter are the amounts of alginite plus liptodetrinite. Vitrinite reflectance values (Ro) in \%.

Table 6. Results from CHN analyses performed on kerogen concentrates from Site 658 sediments.

\begin{tabular}{|c|c|c|c|c|}
\hline $\begin{array}{l}\text { Core, section, } \\
\text { interval }(\mathrm{cm})\end{array}$ & $\begin{array}{c}\text { Nitrogen } \\
\text { (wt\%) }\end{array}$ & $\begin{array}{l}\text { Organic } \\
\text { carbon } \\
\text { (wt\%) }\end{array}$ & $\begin{array}{c}\text { Hydrogen } \\
\text { (wt\%) }\end{array}$ & $\mathrm{H} / \mathrm{C}$ \\
\hline \multicolumn{5}{|l|}{ 108-658A- } \\
\hline $1 \mathrm{H}-2,40$ & 6.12 & 51.56 & 4.11 & 0.96 \\
\hline $3 \mathrm{H}-4,40$ & 3.45 & 44.89 & 5.47 & 1.44 \\
\hline $3 \mathrm{H}-5,40$ & 2.04 & 27.59 & 3.33 & 1.44 \\
\hline $4 \mathrm{H}-6,40$ & 3.39 & 48.94 & 5.76 & 1.41 \\
\hline $5 \mathrm{H}-4,40$ & 1.62 & 21.95 & 3.17 & 1.68 \\
\hline $6 \mathrm{H}-1,59$ & 3.14 & 45.83 & 5.64 & 1.44 \\
\hline $8 \mathrm{H}-3,40$ & 4.37 & 56.08 & 5.47 & 1.17 \\
\hline $10 \mathrm{H}-4,40$ & 2.31 & 39.23 & 4.84 & 1.44 \\
\hline $13 \mathrm{H}-4,40$ & 2.84 & 54.65 & 5.10 & 1.12 \\
\hline $15 \mathrm{H}-4,44$ & 2.81 & 46.61 & 5.6 & 1.44 \\
\hline $16 \mathrm{H}-6,40$ & 2.3 & 39.76 & 4.8 & 1.44 \\
\hline $16 \mathrm{H}-7,40$ & 3.5 & 54.07 & 6.52 & 1.45 \\
\hline $17 \mathrm{H}-2,40$ & 2.66 & 51.90 & 5.65 & 1.31 \\
\hline $17 \mathrm{H}-3,40$ & 5.23 & 58.55 & 6.59 & 1.35 \\
\hline $20 \mathrm{H}-1,40$ & 2.58 & 39.42 & 4.87 & 1.44 \\
\hline $21 \mathrm{H}-1,40$ & 2.98 & 51.43 & 4.25 & 0.99 \\
\hline $21 \mathrm{H}-2,37$ & 2.68 & 46.08 & 5.74 & 1.44 \\
\hline $23 \mathrm{H}-5,40$ & 2.74 & 47.69 & 5.54 & 1.44 \\
\hline $24 \mathrm{H}-3,40$ & 3.1 & 55.91 & 6.48 & 1.39 \\
\hline $25 \mathrm{H}-6,16$ & 2.28 & 41.03 & 4.53 & 1.32 \\
\hline $26 \mathrm{H}-3,76$ & 2.73 & 53.33 & 6.14 & 1.38 \\
\hline $29 \mathrm{H}-5,40$ & 1.81 & 32.92 & 3.96 & 1.44 \\
\hline $30 \mathrm{H}-4,40$ & 1.78 & 30.72 & 4.16 & 1.68 \\
\hline $30 \mathrm{H}-6,87$ & 3.23 & 50.01 & 6.21 & 1.49 \\
\hline $31 \mathrm{H}-2,40$ & 2.39 & 38.41 & 4.23 & 1.32 \\
\hline $31 \mathrm{H}-3,40$ & 2.56 & 46.68 & 5.52 & 1.42 \\
\hline
\end{tabular}

Note: The $\mathrm{H} / \mathrm{C}$ ratio is expressed as atomic hydrogen/carbon ratio.

High accumulation rates may imply intense fluvial runoff and dense vegetation cover in the source area due to humid climatic conditions. On the other hand, during times of arid climatic conditions (such as those typical for northwest Africa today), reduced vegetation cover and the lack of river discharge result in decreased accumulation rates of terrigenous organic matter.
Because these changes in paleoclimate are also indicated by changes in the accumulation rates of the siliciclastic fraction (mainly quartz, clay minerals, and feldspars; cf. Stein, 1985; Tiedemann et al., this vol.), accumulation rates of the organic and inorganic terrigenous fractions are compared in Figure 13 (for pollen data necessary for more quantitative estimates of paleoclimate, see Beug et al., this vol.).

According to the record of the terrigenous sediment fractions at Site 658 , the evolution of climate in northwest Africa during approximately the last $3.7 \mathrm{Ma}$ can be described as follows:

1. Prior to about $3.1 \mathrm{Ma}$, high accumulation rates of organic matter and quartz and nonquartz siliciclastics were recorded (Fig. 13). The supply of terrigenous organic matter reaches maximum values of up to $0.3 \mathrm{gC} \cdot \mathrm{cm}^{-2} \cdot 1000 \mathrm{yr}^{-1}$ between 3.5 and $3.1 \mathrm{Ma}$. This is interpreted as humid climatic conditions dominating in northwest Africa during that time interval, which resulted in increased fluvial supply of organic matter and fine-grained siliciclastics (cf. Sarnthein et al., 1982; Stein, 1985; Tiedemann et al., this vol.). The climatic situation must have been similar to that suggested for interglacials during the Quaternary (Fig. 14A).

2. The interval between 3.1 and $2.45 \mathrm{Ma}$ is characterized by long-term changes in both the organic and inorganic terrigenous sediment supply. The most drastic changes occur at the beginning of this interval, starting with the distinct decrease in organic matter supply at $3.1 \mathrm{Ma}$ and followed by distinct decreases in the supply of quartz at $3 \mathrm{Ma}$ and of nonquartz siliciclastics at $2.8 \mathrm{Ma}$ (Fig. 13; cf. Tiedemann et al., this vol.). The accumulation rates of terrigenous organic matter may reach values as low as $0.04 \mathrm{gC} \cdot \mathrm{cm}^{-2} \cdot 1000 \mathrm{yr}^{-1}$. This interval of major decrease in accumulation rates is interpreted as a transition from dominantly humid to more arid climatic conditions (cf. Sarnthein et al., 1982; Stein, 1985). For a more detailed interpretation of the major nonsynchronous changes of accumulation rates of organic and siliciclastic fractions, further information about the composition of the nonquartz siliciclastic fraction (clay minerals, feldspars, etc.) is needed.

3. Between 2.45 and $1.6 \mathrm{Ma}$, low accumulation rates of terrigenous organic matter and nonquartz (clayey) siliciclas- 

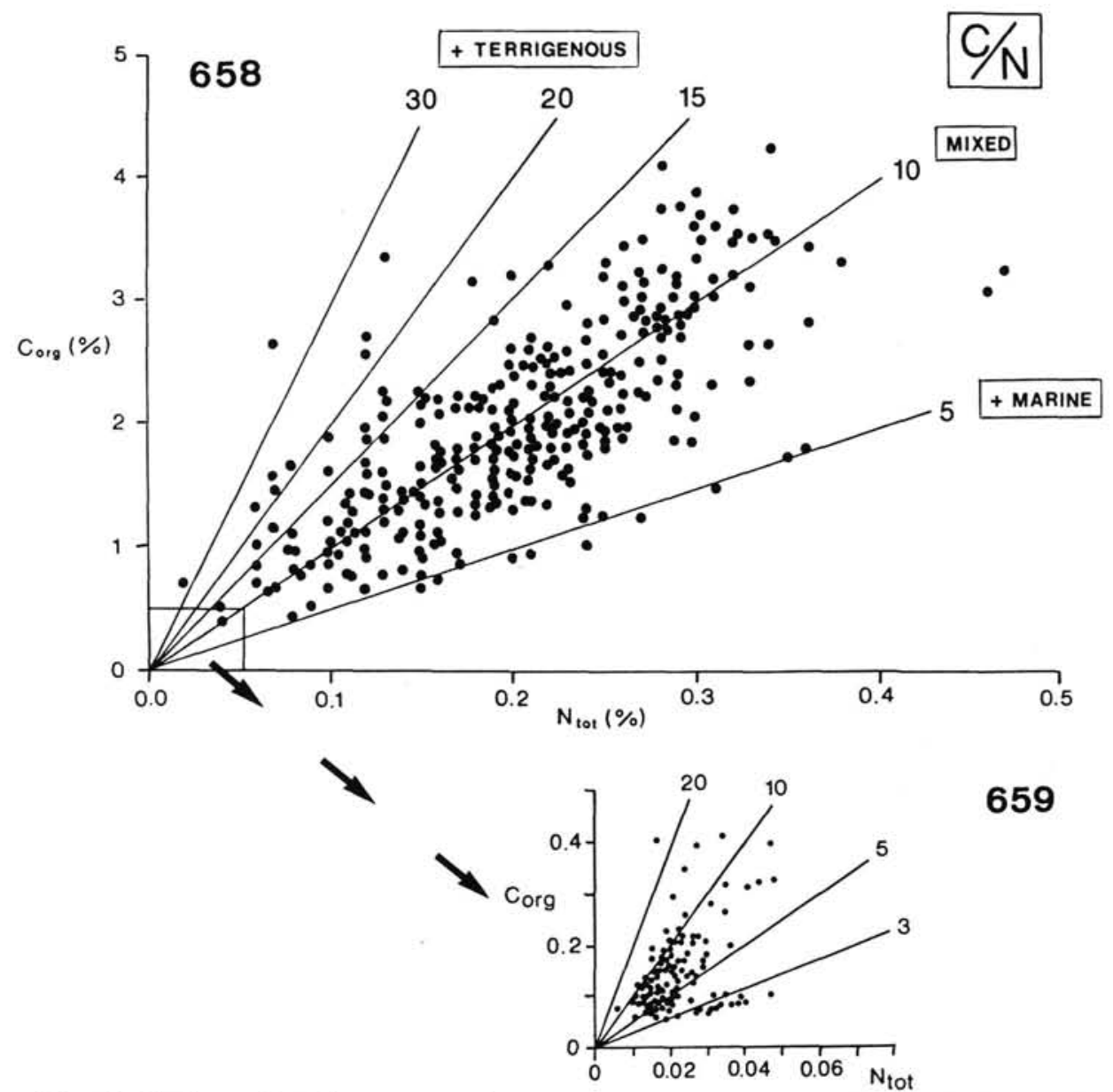

659

Figure 9. Total organic carbon vs. total nitrogen for sediments from Sites 658 and 659. Lines indicate $\mathrm{C} / \mathrm{N}$ ratios (i.e., $3,5,10,15,20$, and 30 ). $\mathrm{C} / \mathrm{N}$ ratios of $>>15$ suggest a higher terrigenous proportion; ratios around 10, a mixed type of organic matter; and ratios around 5, predominantly marine organic matter.

tics indicate reduced fluvial input due to a more arid climate in northwest Africa. On the other hand, increased accumulation rates of quartz were recorded in carbonate-poor (glacial) intervals. This may suggest increased eolian input during glacial times because of increase in aridity (Fig. 14B; cf. Stein, 1985; Tiedemann et al., this vol.). Our interpretation of an increased fluvial supply of quartz between 3 and 3.4 Ma and an increased eolian input that caused the quartz maxima during the younger upper Pliocene/Pleistocene time interval could be verified by grain-size and (possibly) grain-shape analyses of the quartz fractions (Sarnthein and Koopmann, 1980; Mazzullo et al., 1985; Stein, 1985; Tiedemann et al., this vol.).

4. The last $0.7 \mathrm{Ma}$ are characterized by a distinct increase in amplitude of variations between low and high accumulation rates of quartz, nonquartz siliciclastics, and terrigenous organic matter (Fig. 13). This is interpreted as a result of distinct cyclic changes in paleoclimate between arid and humid conditions in northwest Africa. During glacial times, an arid climate was dominant, which caused a reduced fluvial input (i.e., decreased accumulation rates of clay and terrigenous organic matter) and an increased eolian quartz input (Fig. 14B). On the other hand, a more humid climate prevailed during interglacial times, resulting in low accumulation rates of (eolian) quartz and higher rates of supply of (fluvial) clays and terrigenous organic matter (Fig. 14A; cf. Sarnthein and
Koopmann, 1980; Sarnthein et al., 1982; Stein, 1985; Tiedemann et al., this vol).

\section{Marine Organic Carbon: Implications for Paleoproductivity off Northwest Africa}

The accumulation rates of marine organic matter are mainly controlled by the rate of supply (i.e., surface-water productivity) and/or the rate of preservation (Müller and Suess, 1979; Demaison and Moore, 1980; Arthur et al., 1984; Tissot and Welte, 1984). A fully oxygen-deficient environment such as that found in the modern Black Sea and that is characterized as well by a high preservation rate of organic matter (Deuser, 1971; Degens and Ross, 1974) is very unlikely for the northwest African continental margin because bioturbation is common throughout the entire (organic-carbon-rich) sediment sequence at Site 658 (Ruddiman, Sarnthein, et al., 1988). Thus, changes in the accumulation of marine organic carbon at Site 658 are interpreted as a signal mainly of changes in surface-water productivity in the upwelling area off northwest Africa.

High oceanic fertility that causes high productivity off northwest Africa may result from fluvial nutrient supply and/or coastal upwelling of nutrient-rich waters (Fig. 14; Sarnthein et al., 1982; Diester-Haass, 1983; Stein, 1985). Because a high fluvial nutrient supply coincides with an 


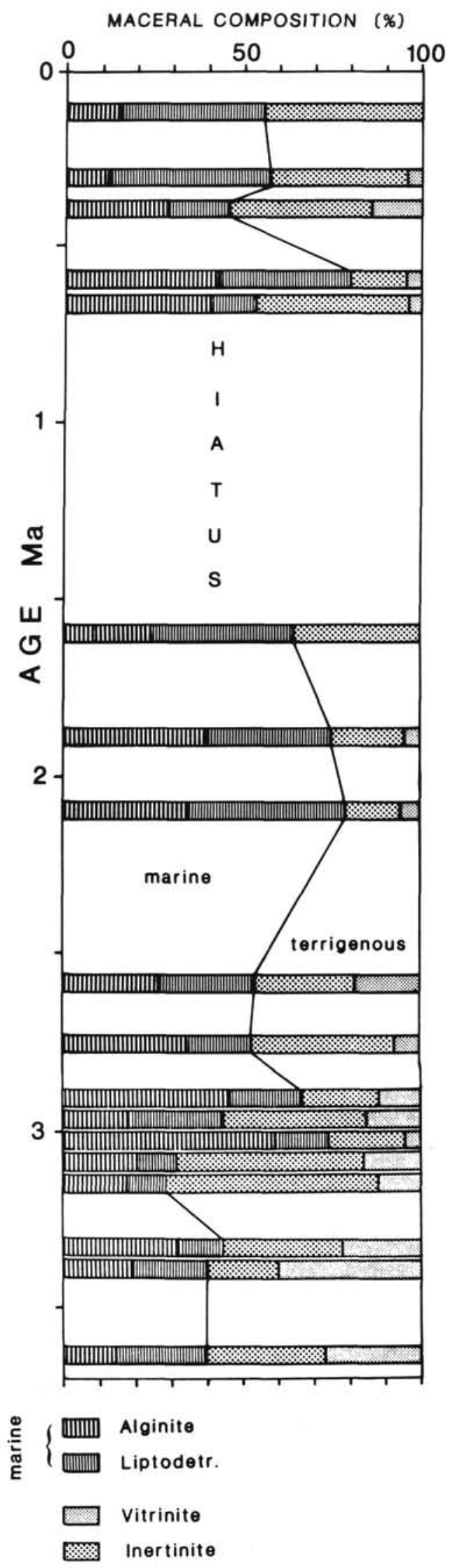

Figure 10. Maceral composition at Site 658 (data from Table 5). For further explanations see text. increased supply of terrigenous organic matter during the (humid) interglacials and increased upwelling occurs during the (arid) glacials (i.e., during times of reduced fluvial input), a comparison of the accumulation rates of marine and terrigenous organic matter may help to distinguish between different mechanisms causing the high productivity (cf. Stein et al., 1986; ten Haven et al., 1987).

In oxic environments, paleoproductivity can be roughly estimated from the accumulation rates of (marine) organic carbon and from a comparison of the (marine) organic carbon content and the sedimentation rate (Figs. 13 and 15; Müller and Suess, 1979; Stein, 1986). From these data, low productivity is typical for open-ocean, nonupwelling Sites 657 and 659. Productivity at these two sites was probably slightly higher during Pliocene-Pleistocene time than during late Miocene time (Fig. 15). On the other hand, at Site 658, which is located inside the upwelling area off northwest Africa, distinctly higher oceanic productivity is obvious. Accumulation rates of marine organic carbon may reach values as high as 0.2 $\mathrm{gC} \cdot \mathrm{cm}^{-2} \cdot 1000 \mathrm{yr}^{-1}$ (Fig. 13). It seems that these maximum values occur during the humid interglacial as well as during the arid glacial stages. In the organic carbon/sedimentation rate plot (Fig. 15), data from Site 658 fall into "high-productivity field" $A$ '. The influence of high productivity is also indicated by increased amounts of biogenic opal recorded in the sediments of Site 658 (Ruddiman, Sarnthein, et al., 1988; Mienert et al., 1988).

For more quantitative estimates of changes in paleoproductivity, equations are available to calculate surface-water productivity from sediment data (Müller and Suess, 1979; Stein, 1986; Sarnthein et al., 1987). In Table 7, mean values of paleoproductivity for different time intervals are summarized for Sites 658 and 659.

At Site 659 , paleoproductivity values vary between 20 and $50 \mathrm{gC} \cdot \mathrm{m}^{-2} \cdot \mathrm{yr}^{-1}$ (Table 7). These values are typical for open-marine environments (Romankevich, 1984). At upwelling Site 658 , the values vary between 160 and $320 \mathrm{gC} \cdot \mathrm{m}^{-2}$ - $\mathrm{yr}^{-1}$ (Table 7), reflecting the position of Site 658 inside a high-productivity area. These paleoproductivity values estimated from the organic carbon data at Site 658 are similar to those measured in the upwelling area off northwest Africa today $\left(150\right.$ to $>325 \mathrm{gC} \cdot \mathrm{m}^{-2} \cdot \mathrm{yr}^{-1}$; Schemainda et al., 1975).

In general, productivity estimated for glacial intervals is very similar to (slightly higher than) that estimated for interglacial intervals. This may imply that a higher fluvial nutrient supply during humid interglacials and a higher nutrient supply from intensified upwelling during arid glacials may have had similar effects on surface-water productivity off northwest Africa (Fig. 14; Diester-Haass, 1983; Stein, 1985).

High productivity values were also estimated for the intervals of carbonate minima between 3.5 and $3.1 \mathrm{Ma}$ (Table 6). During this time, accumulation rates of both marine and terrigenous organic matter reached their maximum values (Fig. 13). The carbonate minima of this interval are interpreted as times of slightly lowered sea-level and semiarid climates in northwest Africa (cf. Stein, 1985). During this time, terrigenous organic matter as well as nutrients could be transported close to the shelf edge. These fluvial nutrients, together with nutrients supplied by upwelled waters, may have resulted in increased productivity in surface waters near the shelf edge/upper slope area (i.e., closer to the location of Site 658).

For determining more exactly the relationships between paleoclimate in northwest Africa and paleoproductivity in the eastern subtropical Atlantic (i.e., especially the leads and lags of the different signals), a high-resolution study on sediments of Site 658 , including stable isotope and pollen data, should be 
Table 7. Estimates of paleoproductivity for different time intervals.

\begin{tabular}{|c|c|c|c|c|c|c|c|c|}
\hline \multirow{2}{*}{$\begin{array}{l}\text { Depth } \\
\text { (mbsf) }\end{array}$} & \multirow[b]{2}{*}{ Age (Ma) } & \multirow{2}{*}{$\begin{array}{c}\text { Wet-bulk } \\
\text { density } \\
(\%)\end{array}$} & \multirow{2}{*}{$\begin{array}{c}\text { Porosity } \\
\text { (\%) }\end{array}$} & \multirow{2}{*}{$\begin{array}{c}\text { Mean } \\
\text { sed. rate } \\
(\%)\end{array}$} & \multirow{2}{*}{$\begin{array}{c}\text { Total } \\
\text { organic } \\
\text { content }\end{array}$} & \multirow{2}{*}{$\begin{array}{l}\text { Marine } \\
\text { organic } \\
\text { content }\end{array}$} & \multicolumn{2}{|c|}{$\begin{array}{l}\text { Paleopro- } \\
\text { ductivity }\end{array}$} \\
\hline & & & & & & & I & $\overline{\text { II }}$ \\
\hline \multicolumn{9}{|l|}{ Site 658: } \\
\hline $0-100$ & $0-0.7$ & 1.65 & 68 & 14.7 & a: 1.61 & $\begin{array}{l}1.05 \\
0.82\end{array}$ & $\begin{array}{l}190 \\
160\end{array}$ & $\begin{array}{l}150 \\
130\end{array}$ \\
\hline \multirow[t]{2}{*}{$100-174$} & $1.6-1.45$ & 1.60 & 62 & 7.2 & a: 2.13 & 1.38 & 240 & 210 \\
\hline & & & & & b: 1.81 & 1.18 & 220 & 190 \\
\hline \multirow[t]{2}{*}{$174-240$} & $2.45-3.1$ & 1.60 & 62 & 10.8 & a: 1.99 & 1.29 & 240 & 200 \\
\hline & & & & & b: 1.67 & 1.09 & 210 & 170 \\
\hline \multirow[t]{2}{*}{$240-295$} & $3.1-3.6$ & 1.73 & 56 & 10.8 & a: 2.54 & 1.65 & 320 & 260 \\
\hline & & & & & b: 1.49 & 0.97 & 220 & 180 \\
\hline \multicolumn{9}{|l|}{ Site 659: } \\
\hline $0-50$ & $0-1.6$ & 1.51 & 68 & 3 & 0.22 & 0.15 & 40 & 50 \\
\hline $50-135$ & $1.5-4.5$ & 1.6 & 64 & 3 & 0.15 & 0.1 & 30 & 40 \\
\hline $135-230$ & $4.5-8.5$ & 1.74 & 56 & 0.3 & 0.07 & 0.05 & 20 & 30 \\
\hline
\end{tabular}

Note: For the time interval from 0 to $2.45 \mathrm{Ma}$, a (from intervals with minimum carbonate content) is interpreted as glacial, and b (from intervals with maximum carbonate content), as interglacial stages. I = Paleoproductivity values calculated according to Stein (1986a); for comparison, productivity values (II) calculated according to Sarnthein et al. (1987) are shown. Wet-bulk density, porosity, mean sedimentation rates, and water depths (Site 658: $2260 \mathrm{~m}$; Site 659: 3070 $\mathrm{m})$ required for calculation of paleoproductivity are from Ruddiman, Sarnthein, et al. (1988). The amount of marine organic carbon is shown as a percent of the whole sample. For further explanations, see "Methods" section, this chapter.

added to this more general study, which has concentrated on the long-term changes.

\section{Organic-Carbon-Rich Sediments at Site 657: Implications for Turbidites and Slumping at the Northwest African Margin}

At Site 657, normal pelagic sedimentation is often interrupted by events of turbidite flows and slumping processes (Faugères et al., this vol.). A few of these events are also documented in the sediment samples investigated for organic carbon content. In general, organic carbon contents for the sediments at Site 657 are very low, as is typical for open-marine environments. The samples from the turbidites and the slump, however, have very high TOC values of $1 \%-3 \%$ (Fig. 5). Such phenomena are also reported for adjacent deep-sea basins in the eastern subtropical Atlantic (e.g., Weaver and Kuijpers, 1983; Colley et al., 1984).

The quality of the organic matter in these redeposited sediments is very similar to that found in the organic-carbon-rich sediments at Site 658, as indicated by Rock-Eval and organic carbon/nitrogen data (Fig. 7 and Table 3). Thus, the source area of both these turbidites and the slump is very likely to be the upwelling-influenced, high-productivity area of the upper slope (Fig. 14). Because the organic matter in turbidites and slumps has been rapidly buried after redeposition in the deep sea, the residence time in zones of bioturbation and oxic decomposition is short. In this way, higher amounts of (marine) organic matter can also be preserved in an oxygen-rich, open-marine environment (cf. Jones, 1983; Arthur et al., 1984). In the upper parts of such (turbiditic) layers, the organic matter can be remineralized by post-depositional oxidation fronts (cf. Colley et al., 1984), which should be recognizable in a detailed investigation of the turbidite sequences.

\section{CONCLUSIONS}

The results of our detailed organic geochemical study of sediments from Sites 657, 658, and 659 can be summarized as follows:

1. The sediments of nonupwelling Sites 657 and 659 display low organic carbon values of less than $0.5 \%$, values typical for normal open-marine environments. In four turbiditic and slumping sequences at Site 657 , high TOC values of $1 \%-3 \%$ have been recorded.

2. At upwelling Site 658 , high TOC values of $0.5 \%-4 \%$ occur. Short-term fluctuations in organic carbon with periods between about 20,000 and 100,000 yr (i.e., "Milankovitch-type" cycles) are typical, suggesting that changes in climate are the mechanisms for these variations. High hydrogen index values, low $\mathrm{C} / \mathrm{N}$ ratios, and high amounts of alginite and liptodetrinite imply that the organic carbon is a mixture of marine and terrigenous matter (i.e., a kerogen type II/III), with a dominance of the marine portion.

3. At open-marine nonupwelling Site 659 , mean paleoproductivity varies between 20 and $50 \mathrm{gC} \cdot \mathrm{m}^{-2} \cdot \mathrm{y}^{-1}$. At upwelling Site 658 , mean productivity is distinctly higher $\left(160-320 \mathrm{gC} \cdot \mathrm{m}^{-2} \cdot \mathrm{y}^{-1}\right)$. The changes in productivity at Site 658 are controlled by changes in the nutrient supply from upwelled waters and in the fluvial nutrient supply.

4. The distinct decrease in accumulation rates of terrigenous sediment fractions at Site 658 between 3.1 and $2.45 \mathrm{Ma}$ indicates the change from a dominantly humid climate prior to $3.1 \mathrm{Ma}$ to a climate of arid/humid (semiarid) cycles typical of the last $2.45 \mathrm{Ma}$. During the last $0.7 \mathrm{Ma}$, the amplitude of these climatic cycles distinctly increased, indicated by high-amplitude variations of accumulation rates of organic carbon and siliciclastic sediment fractions.

5. Vitrinite reflectance values of $0.23 \%-0.27 \%$ and low $\mathrm{T}_{\max }$ values of $400^{\circ} \mathrm{C}-425^{\circ} \mathrm{C}$ indicate the immaturity of organic matter at Site 658 .

\section{ACKNOWLEDGMENTS}

We would like to thank E. Biermanns, M. Dersch, F. Leistner, U. Mann, D. Ortmann, and R. Stax for technical assistance. We thank Drs. W. Ruddiman and E. Suess for their numerous constructive suggestions for improvement of the manuscript. Financial support by the Deutsche Forschungsgemeinschaft is gratefully acknowledged (Grants No. STE 412/1 and No. WE 346/27).

\section{REFERENCES}

Arthur, M. A., Dean, W. E., and Stow, D.A.V., 1984. Models for the deposition of Mesozoic-Cenozoic fine-grained organic-carbon-rich sediment in the deep sea. In Stow, D.A.V., and Piper, D.J.W. (Eds.), Fine-Grained Sediments: Deep-Water Processes and Facies. Geol. Soc. Spec. Publ. (London), 15:527-560.

Betzer, P. R., Showers, W. J., Laws, E. A., Winn, C. D., Ditullio, G. R., and Kroopnick, P. M., 1984. Primary productivity and particle fluxes on a transect of the equator at $153^{\circ} \mathrm{W}$ in the Pacific Ocean. Deep-Sea Res., 31:1-11.

Bordowskiy, O. K., 1965a. Accumulation of organic matter in bottom sediments. Mar. Geol., 3:33-82. 
O. K., 1965b. Transformation of organic matter in bottom sediments and its early diagenesis. Mar. Geol., 3:83-114.

Colley, S., Thompson, J., Wilson, T.R.S., and Higgs, N. C., 1984. Post-depositional migration of elements during diagenesis in brown clay and turbidite sequences in the northeast Atlantic. Geochim. Cosmochim. Acta, 48:1223-1235.

Cook, H. E., Johnson, P. D., Matti, J. C., and Zemmels, I., 1975. Methods of sample preparation and X-ray diffraction data analysis, X-ray mineralogy laboratory, Deep Sea Drilling Project, University of California, Riverside. In Hayes, D. E., Frakes, L. A., et al., Init. Repts. DSDP, 28: Washington (U.S. Printing Office), 999-1007.

Degens, E. T., and Ross, D. A., eds., 1974. The Black Sea-Geology, Chemistry, and Biology. AAPG Mem., 20.

Demaison, G. J., and Moore, G. T., 1980. Anoxic environments and oil source bed genesis. Org. Geochem., 2:9-31.

Deuser, W. G., 1971. Organic-carbon budget of the Black Sea. Deep-Sea Res., 18:995-1004.

Diester-Haass, L., 1983. Differentiation of high oceanic fertility in marine sediments caused by coastal upwelling and/or river discharge off northwest Africa during the late Quaternary. In Thiede, J., and Suess, E. (Eds.), Coastal Upwelling: Its Sediment Record. NATO Conf. Series IV: New York (Plenum Press), 10B:399-419.

Durand, B., ed., 1980. Kerogen: Insoluble Organic Matter from Sedimentary Rocks: Paris (Editions Technip).

Espitalié, J., Laporte, J. L., Madec, M., Marquis, F., Leplat, P., Paulet, J., and Boutefeu, A., 1977. Methode rapide de characterisation des roches-mere, de leur potential petrolier et de leur degre d'evolution. Rev. Inst. Fr. Pet., 32:23-42.

Hutton, A. C., Kantsler, A. J., Cook, A. C., and Mckirdy, D. M., 1980. Organic matter in oil shales. Aust. Pet. Explor. Assoc. J., 20:44-67.

Jones, R. W., 1983. Organic matter characteristics near the shelfslope boundary. SEPM Spec. Publ., 33:391-405.

Littke, R., 1987. Petrology and genesis of upper Carboniferous seams from the Ruhr region, West Germany. Int. J. Coal Geol., $7: 147-184$.

McIver, R., 1975. Hydrocarbon occurrence from JOIDES Deep Sea Drilling Project. Proc. World Petrol. Congr., 9:269-280.

Mazzullo, J., Sims, D., and Cunningham, D., 1985. The effects of eolian sorting abrasion upon the shapes of fine quartz sand grains. J. Sediment. Petrol., 56:45-56.

Mienert, J., Stein, R., Schultheiss, P., and Shipboard Scientific Party, 1988. Relationship between grain density and biogenic opal in sediments from Sites 658 and 660 . In Ruddiman, W., Sarnthein, M., et al. (Eds.), Proc. ODP, Init. Repts., 108: College Station, TX (Ocean Drilling Program), 1047-1053.

Müller, P. J., 1977. C/N ratios in Pacific deep-sea sediments: effect of inorganic ammonium and organic nitrogen compounds sorbed by clays. Geochim. Cosmochim. Acta, 41:765-776.

Müller, P. J., and Suess, E., 1979. Productivity, sedimentation rate and sedimentary organic matter in the oceans. I. Organic matter preservation. Deep-Sea Res., 26:1347-1362.

Romankevich, E. A., 1984. Geochemistry of Organic Matter in the Ocean: Berlin-Heidelberg-New York (Springer-Verlag).

Ruddiman, W., Sarnthein, M., et al., 1988. Proc. ODP, Init. Repts, 108: College Station, TX (Ocean Drilling Program).

Rullkötter, J. and Mukhopadhyay, P. K., 1986. Comparison of Mesozoic carbonaceous claystones in the western and eastern North Atlantic (DSDP Legs 76, 79 and 93). In Summerhayes, C. P., and Shackleton, N. J. (Eds.), North Atlantic Paleoceanography. Geol. Soc. Spec. Publ. (London), 21:377-387.

Rullkötter, J., Mukhopadhyay, P. K., Schaefer, R. G., and Welte, D. H., 1984. Geochemistry and petrography of organic matter in sediments from Deep Sea Drilling Project Sites 545 and 547, Mazagan Escarpment. In Hinz, K., Winterer, E. L., et al., Init. Repts. DSDP, 79: Washington (U.S. Govt. Printing Office), 775806.

Sarnthein, M., and Koopmann, B., 1980. Late Quaternary deep-sea record on northwest African dust supply and wind circulation. Palaeoecol. Africa, 12:239-253.

Sarnthein, M., Koopmann, B., Wolter, K., and Pflaumann, U., 1981. Glacial and interglacial wind regimes over the eastern subtropical Atlantic and northwest Africa. Nature, 293:193-196.
Sarnthein, M., Thiede, J., Pflaumann, U., Erlenkeuser, H., Fütterer, D., Koopmann, B., Lange, H., and Seibold, E., 1982. Atmospheric and oceanic circulation patterns off northwest Africa during the past 25 million years. In von Rad, U., Hinz, K., Sarnthein, M., and Seibold, E. (Eds.), Geology of the Northwest African Continental Margin: Berlin-Heidelberg-New York (Springer-Verlag), 545-603.

Sarnthein, M., Winn, K., and Zahn, R., 1987. Paleoproductivity of oceanic upwelling and the effect on atmospheric $\mathrm{CO}_{2}$ and climatic change during deglaciation times. In Berger, W. H., and Labeyrie, L. D. (Eds.), Abrupt Climatic Change: Hingham, MA (D. Reidel), 311-337.

Scheffer, F., and Schachtschabel, P., 1984. Lehrbuch der Bodenkunde: Stuttgart (F. Enke Verlag).

Schemainda, R., Nehring, D., and Schulz, S., 1975. Ozeanologische Untersuchungen zum Produktionspotential der nordwestafrikanischen Wasserauftriebsregion, 1970-1973. Geod. Geophys. Veröff., 4 (16): $1-88$.

Schlanger, S. O., and Jenkyns, H. C., 1976. Cretaceous oceanic anoxic event: causes and consequences. Geol. Mijnbouw, 55:179-184.

Shaffer, G., 1976. A mesoscale study of coastal upwelling variability off northwest Africa. Meteor Forschungsergeb., Reihe A, 17:33-70.

Stach, E., Mackowsky, M. T., Teichmüller, M., Taylor, G. H., Chandra, D., and Teichmüller, R., 1982. Stach's Textbook of Coal Petrology: Berlin (Borntraeger Verlag).

Stein, R., 1985. Late Neogene changes of paleoclimate and paleoproductivity off northwest Africa (DSDP Site 397). Palaeogeogr., Palaeoclimatol., Palaeoecol., 49:47-49.

1986. Surface-water paleo-productivity as inferred from sediments deposited in oxic and anoxic deep-water environments of the Mesozoic Atlantic Ocean. In Degens, E. T., et al. (Eds.), Biochemistry of Black Shales. Mitt. Geol. Paläont. Inst. Univ. Hamburg, 60:55-70.

1986b. Organic carbon and sedimentation rate-further evidence for anoxic deep-water conditions in the Cenomanian/Turonian Atlantic Ocean. Mar. Geol., 72:199-209.

Stein, R., Littke, R., Stax, R., and Welte, D. H., 1989. Quantity, provenance, and maturity of organic matter at ODP Sites 645, 646, and 647: implications for reconstruction of paleoenvironments in Baffin Bay and Labrador Sea during Tertiary times. In Arthur, M. A., Srivastava, S. P., et al. (Eds.), Proc. ODP, Sci. Results, 105: College Station, TX (Ocean Drilling Program), 185-208.

Stein, R., Rullkötter, J., and Welte, D. H., 1986. Accumulation of organic-carbon-rich sediments in the Late Jurassic and Cretaceous Atlantic Ocean-a synthesis. Chem. Geol., 56:1-32.

Stevenson, F. J., and Cheng, C. N., 1972. Organic geochemistry of the Argentine Basin sediments: carbon-nitrogen relationships and Quaternary correlations. Geochim. Cosmochim. Acta, 36: 653-671.

Summerhayes, C., 1986. Organic-rich Cretaceous sediments from the North Atlantic. In Brooks, J., and Fleet, A. J. (Eds.), Marine Petroleum Source Rocks. Geol. Soc. Spec. Publ. (London), 26:301-316.

ten Haven, H. L., Baas, M., de Leeuw, J. W., Schenck, P. A., and Brinkhuis, H., 1987. Late Quaternary Mediterranean sapropels. II. Organic geochemistry and palynology of S1 sapropels and associated sediments. Chem. Geol., 64:149-167.

Thiede, J., and Suess, E., eds., 1983. Coastal Upwelling: Its Sediment Record. Part B, Sedimentary Records of Ancient Coastal Upwelling. NATO Conf. Series IV (Vol. 10): New York (Plenum Press).

Tissot, B., Deroo, G., and Herbin, J. P., 1979. Organic matter in Cretaceous sediments of the North Atlantic: contribution to sedimentology and paleoceanography. In Talwani, M., Hay, W., Ryan, W.B.R. (Eds.), Deep Drilling Results in the Atlantic Ocean: Continental Margin and Paleoenvironment: Washington (American Geophysical Union), 3:362-374.

Tissot, B., and Welte, D. H., 1984. Petroleum Formation and Occurrence: Berlin-Heidelberg-New York (Springer-Verlag).

Tomczak, M., and Hughes, P., 1980. Three-dimensional variability of water masses and currents in the Canary current upwelling region. Meteor Forschungsergeb., Reihe A, 21:1-24. 
van Andel, T. H., Heath, G. R., and Moore, T. C., 1975. Cenozoic history and paleoceanography of the central equatorial Pacific Ocean. Mem. Geol. Soc. Am., 143.

Waples, D. W., 1983. Reappraisal of anoxia and organic richness, with emphasis on Cretaceous of North Atlantic. AAPG Bull., 61:963-978.
Weaver, P.P.E., and Kuijpers, A., 1983. Climatic control of turbidites deposition on the Madeira Abyssal Plain. Nature, 306:360-363.

Date of initial receipt: 20 April 1988

Date of acceptance: 19 December 1988

Ms 108B-143

$658 \mathrm{~A}-21-6,7-14 \mathrm{~cm}$

"TOTAL LIPIDS"

UNSATURATED KETONES

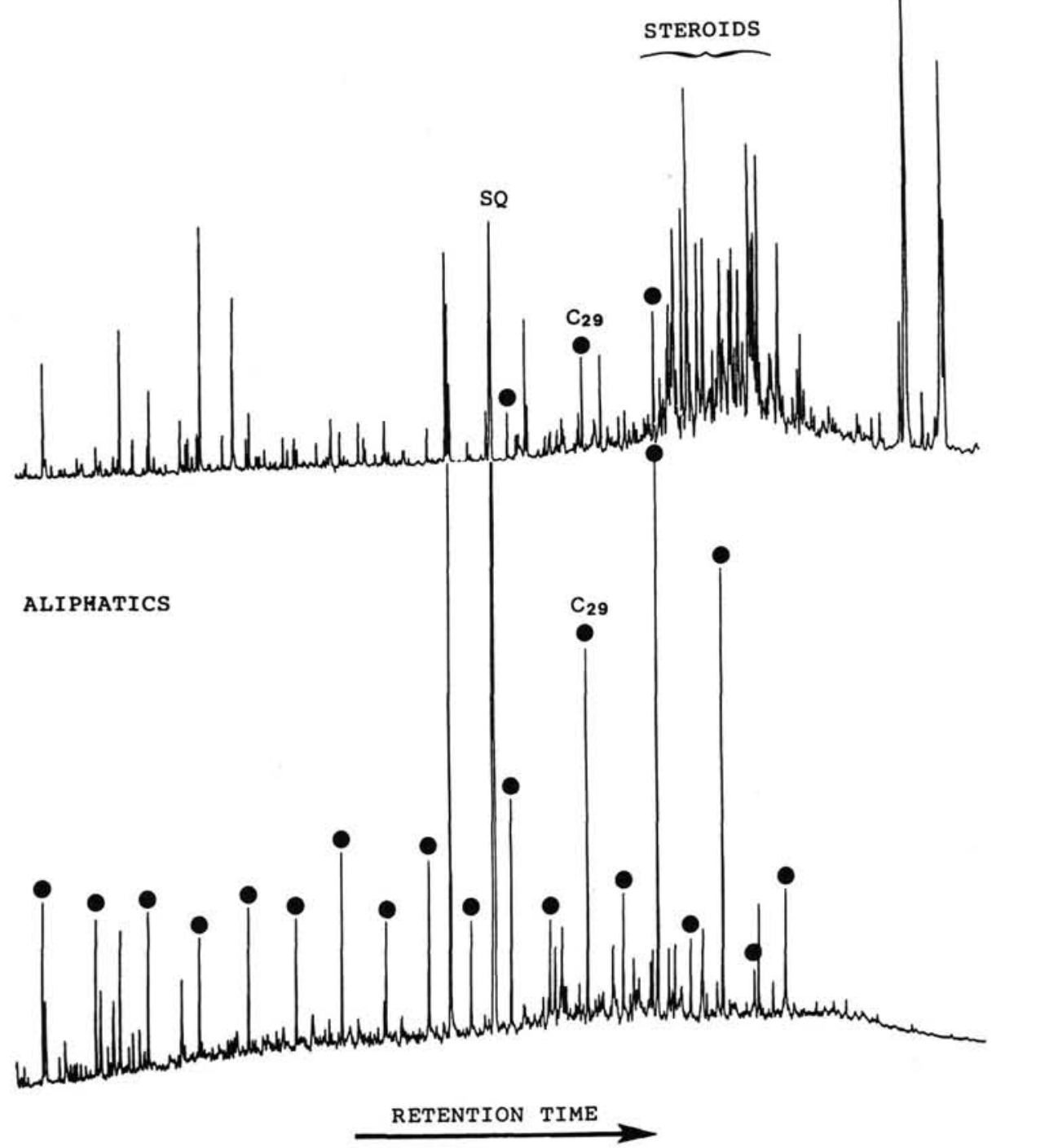

Figure 11. Gas chromatogram of "total lipid" and of aliphatic fraction from Sample 108-658A-21-6, 7-14 $\mathrm{cm}(183 \mathrm{mbsf}) . n$-Alkanes are indicated by dots, starting with $\mathrm{C}_{17} . \mathrm{Sq}=$ squalane; internal standard. The "total lipid" extract was derivatized before GC measurements (see ten Haven, et al., this vol.). 


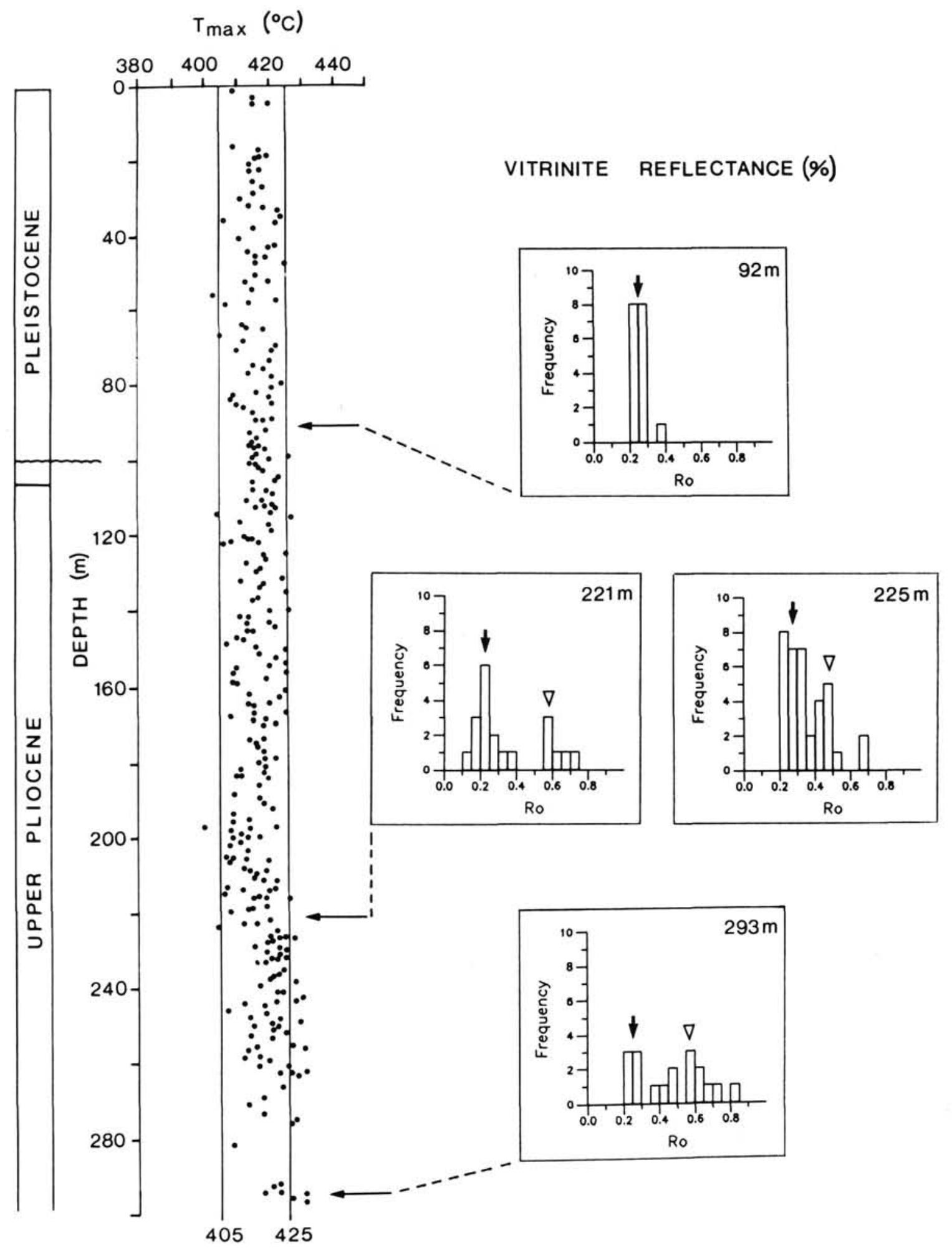

Figure 12. Temperatures of maximum pyrolysis yield $\left(T_{\max }\right)$ from Rock-Eval measurements on sediments from Site 658. Vitrinite reflectance histograms are shown for four selected samples on the right-hand side. 


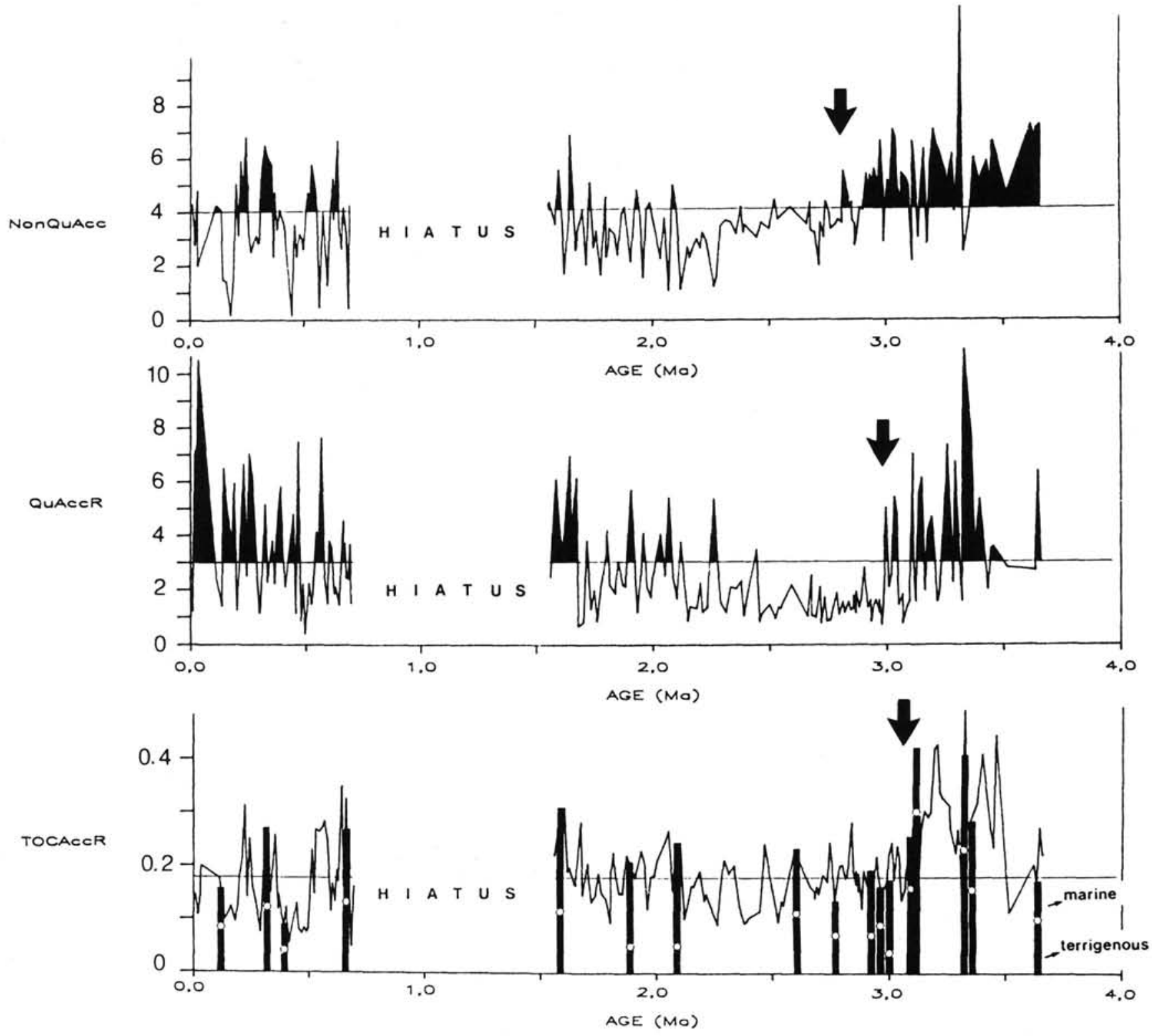

Figure 13. Accumulation rates of organic carbon (bottom), quartz (middle), and nonquartz siliciclastics (top) in $\mathrm{g} \cdot \mathrm{cm}^{-2} \cdot 1000 \mathrm{yr} \mathrm{at}^{-1}$ Site 658. Based on the kerogen microscopy data, accumulation rates of terrigenous and marine organic carbon are separated in record (A). Black arrows indicate times of major changes in accumulation rates. Accumulation rates were calculated using the detailed sedimentation rate data of Sarnthein and Tiedemann (this vol.). 


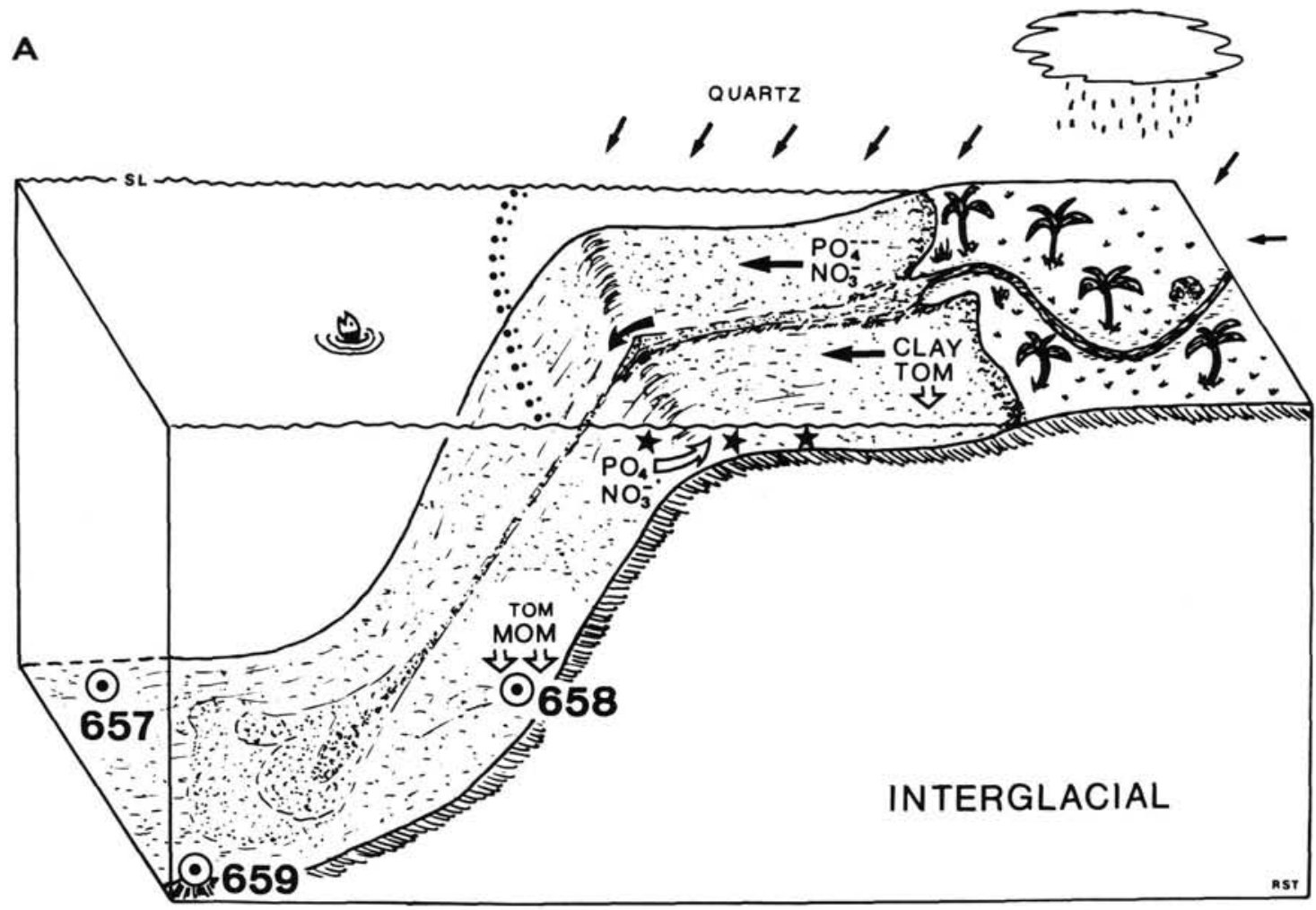

B

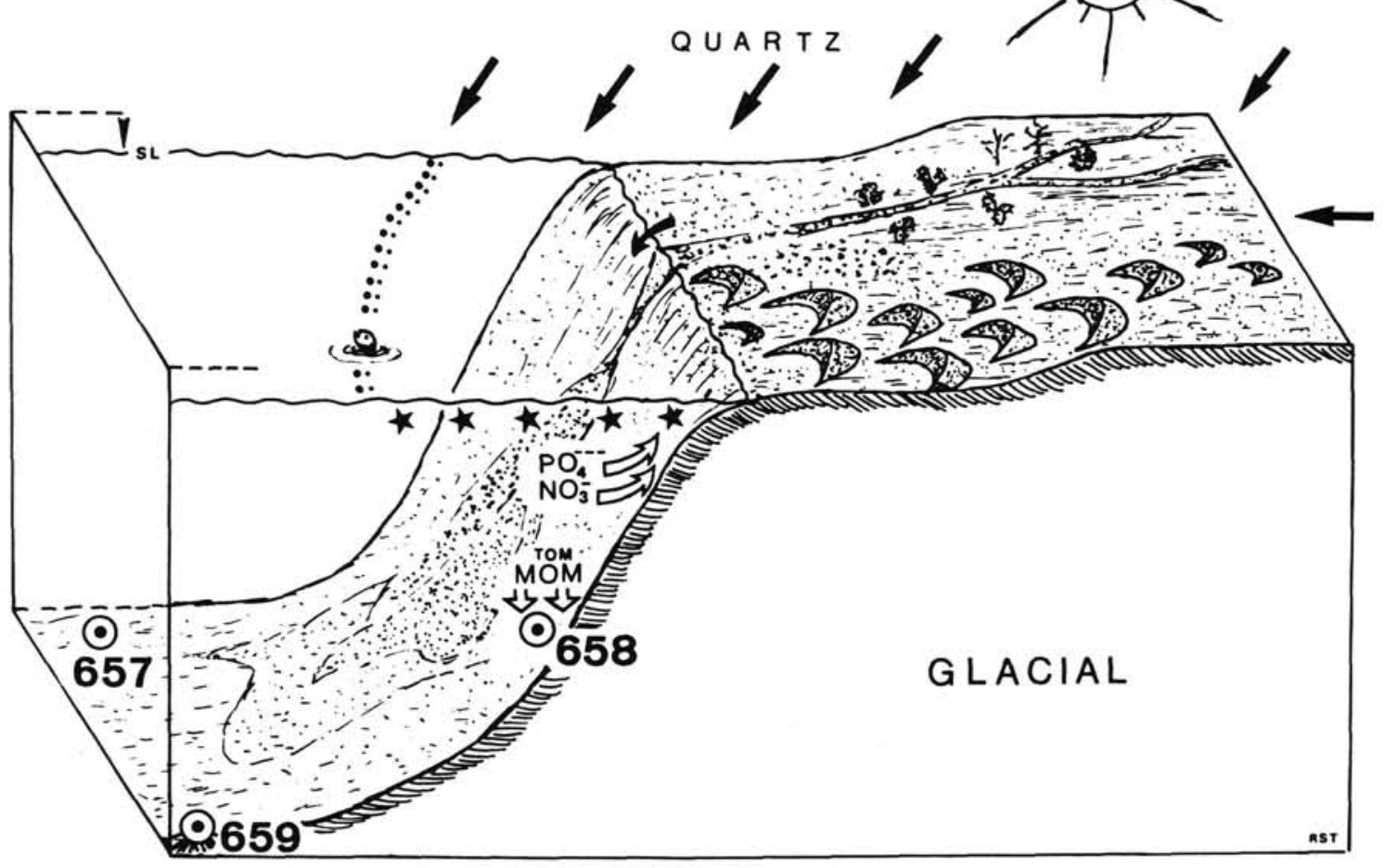

Figure 14. Depositional model for Pliocene-Pleistocene sediments at the northwest African continental margin. A. Interglacial stage. B. Glacial stage. TOM $(\mathrm{MOM})=$ terrigenous (marine) organic matter; $\swarrow=$ northeast trades (increased during glacials, reduced during interglacials); $\diamond=$ upwelling (intensified during glacials, reduced during interglacials); $\leftarrow$ $=$ fluvial input of clay fraction and nutrients (ceased during glacials because of arid climate, increased during interglacials because of humid climate); $\star \star \star=$ area of high oceanic productivity. (For relationships between climate in northwest Africa, trade wind intensity, and upwelling intensity, see Sarnthein et al., 1981, 1982; Stein, 1985). 


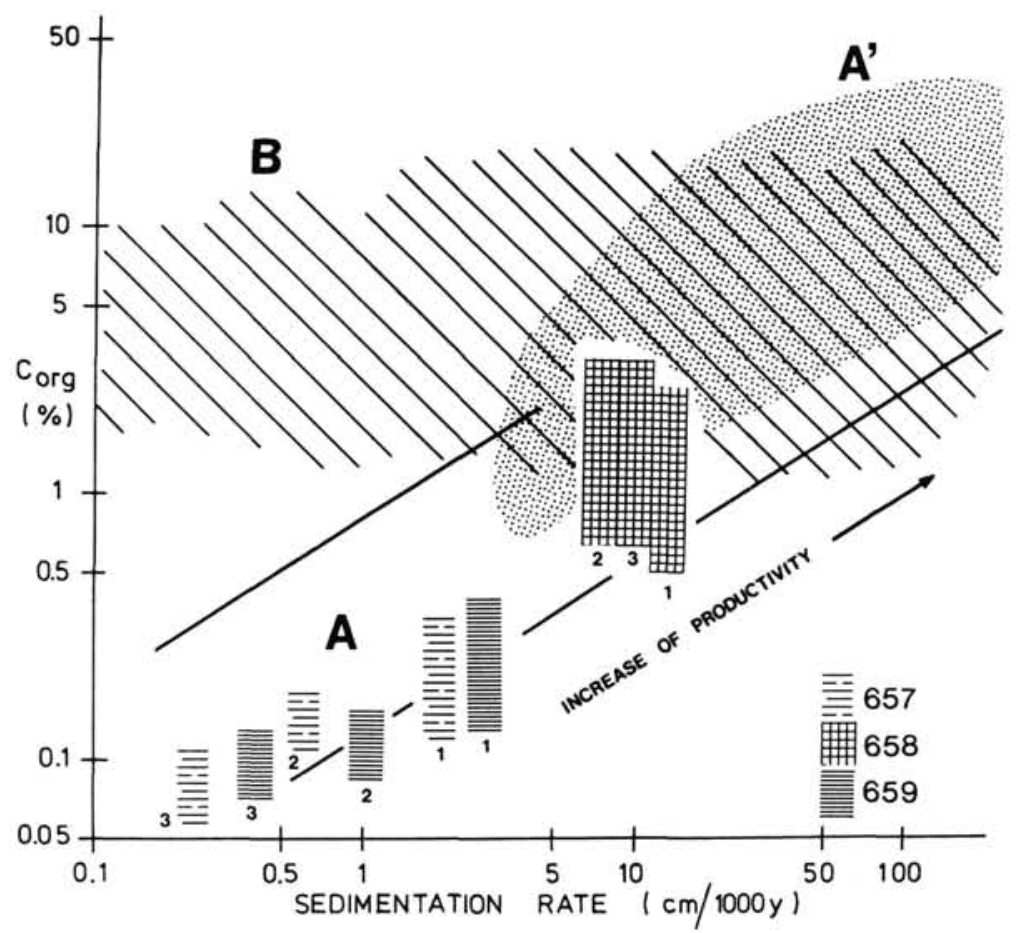

Figure 15. Correlation between (marine) organic carbon values and sedimentation rates. The distinction between fields $\mathrm{A}, \mathrm{A}^{\prime}$, and $\mathrm{B}$ is based on data from Holocene to Miocene sediments (Müller and Suess, 1979; Stein, 1986b). Field A marks positive correlation between organic carbon content and sedimentation rates under oxic deep-water conditions. Field $\mathrm{A}^{\prime}$ indicates conditions of high oceanic productivity, characterized by high organic carbon contents and high sedimentation rates. In general, increased sedimentation rates coincide with increased productivity. Field B indicates data derived from samples deposited under anoxic deep-water conditions. Further details in Stein (1986b). Site 657: (1) Pliocene-Pleistocene (0-4.5 Ma); (2) latest Miocene (6-7 Ma); and (3) late Miocene (7-8.5 Ma). Site 658: (1) Pleistocene (0-0.7 Ma); (2) latest Pliocene (1.6-2.45 Ma); and (3) late Pliocene $(2.45-3.6 \mathrm{Ma})$. Site 659: (1) PliocenePleistocene (0-4.5 Ma); (2) late Miocene (4.5-8.5 Ma); and (3) late Miocene (8.5-10 Ma). 